\title{
O MÉTODO DA FUNÇÃO LAGRANGIANA BARREIRA MODIFICADA/PENALIDADE
}

\section{Aguinaldo Aparecido Pereira}

Dissertação apresentada à Escola de Engenharia de São Carlos, da Universidade de São Paulo, como parte dos requisitos para obtenção do título de Mestre em Engenharia Elétrica.

ORIENTADOR: Prof. Dr. Geraldo Roberto Martins da Costa

São Carlos

2007 
Aos meus pais, Palmira e Sebastião, e a Deus por me darem a dádiva da vida. 
Confia no SENHOR e faze o bem; habitarás na terra, e verdadeiramente serás alimentado. Deleita-se também no SENHOR, e te concederá os desejos do teu coração. Entrega o teu caminho ao SENHOR; confia nele, e ele o fará. E ele fará sobressair a tua justiça como a luz, e o teu juízo como o meio dia. 


\section{AGRADECIMENTOS}

Ao Professor Dr. Geraldo Roberto Martins da Costa pela excelente orientação, compreensão, amizade, paciência e confiança durante a elaboração desse trabalho.

À Professora Dra. Edméa Cássia Baptista pela excelente co-orientação, paciência e principalmente pela amizade que se firmou durante a elaboração desse trabalho.

Ao Professor Dr. Antonio Roberto Balbo, por ter plantado a semente em mim em relação ao mestrado, além do seu incentivo e da sua amizade que vem desde a graduação.

Ao pessoal do LASEE, em especial à Vanusa Alves Sousa, pela troca de idéias, pelo apoio e pela valorosa amizade.

A todos os colegas, professores e funcionários do Departamento de Engenharia Elétrica da EESC/USP pela colaboração.

A “TODOS" os que conviveram comigo durante esse período, torcendo, apoiando, e me compreendendo, muito obrigado!!! 


\section{RESUMO}

PEREIRA, A. A. (2007). O Método da Função Lagrangiana Barreira Modificada/Penalidade. Dissertação (Mestrado) - Escola de Engenharia de São Carlos, Universidade de São Paulo, São Carlos, 2007.

Neste trabalho propomos uma abordagem que utiliza o método de barreira modificada/penalidade para a resolução de problemas restritos gerais de otimização. Para isso, foram obtidos dados teóricos, a partir de um levantamento bibliográfico, que explicitaram os métodos primal-dual barreira logarítmica e método de barreira modificada. Nesta abordagem, as restrições de desigualdade canalizadas são tratadas pela função Barreira de Frisch Modificada, ou por uma Extrapolação Quadrática e as restrições de igualdade do problema através da função Lagrangiana. A implementação consiste num duplo estágio de aproximação: um ciclo externo, onde o problema restrito é convertido em um problema irrestrito, usando a função Lagrangiana Barreira Modificada/Penalidade; e um ciclo interno, onde o método de Newton é utilizado para a atualização das variáveis primais e duais. Ë apresentada também uma função Barreira Clássica Extrapolada para a inicialização dos multiplicadores de Lagrange. A eficiência do método foi verificada utilizando um problema teste e em problemas de Fluxo de Potência Ótimo (FPO).

Palavras-chave: método de pontos interiores, método de barreira modificada, método de Newton, extrapolação quadrática, FPO. 


\section{ABSTRACT}

PEREIRA, A. A. (2007). The Penalty/Modified Barrier Lagrangian Function Method. Dissertation (Master’s degree) - Escola de Engenharia de São Carlos, Universidade de São Paulo, São Carlos, 2007.

In this paper, we propose an approach that utilizes the penalty/modified barrier method to solve the general constrained problems. On this purpose, theoretical data were obtained, from a bibliographical review, which enlightened the logarithmic barrier primal-dual method and modified barrier method. In this approach, the bound constraints are handled by the modified log-barrier function, or by quadratic extrapolation and the equality constraints of the problem through Lagrangian function. The method, as implemented, consists of a two-stage approach: an outer cycle, where the constrained problem is transformed into unconstrained problem, using Penalty/Modified Barrier Lagrangian function; and an inner cycle, where the Newton's method is used for update the primal and dual variables. Also, it is presented a Classical Barrier Extrapolated function for initialization of Lagrange multipliers. The effectiveness of the proposed approach has been examined by solving a test problem and optimal power flow problems (OPF).

Keywords: Interior Point Method, Modified Barrier Method, Newton' Method, quadratic extrapolation, OPF. 


\section{SUMÁRIO}

LISTA DE FIGURAS

LISTA DE TABELAS

xi

LISTA DE ABREVIATURAS E SIGLAS xiii

LISTA DE SÍMBOLOS $\mathrm{XV}$

1 INTRODUÇÃO 1

2 HISTÓRICO 4

3 MÉTODOS DA FUNÇÃO BARREIRA E DA FUNÇÃO BARREIRA MODIFICADA

3.1- O método primal-dual barreira logarítmica. .14

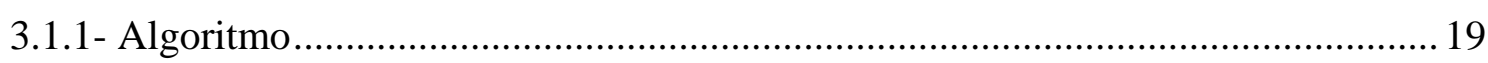

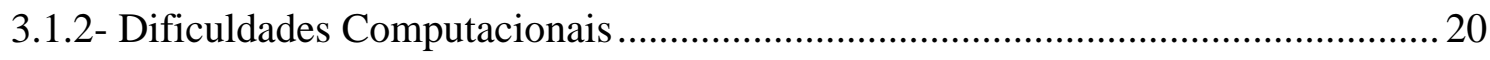

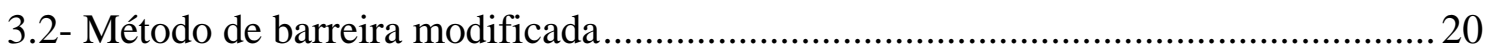

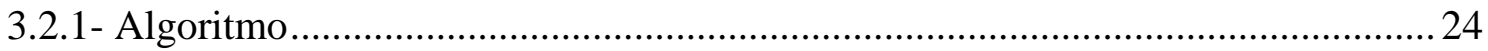

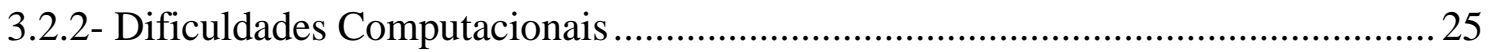

4 O MÉTODO DA FUNÇÃO LAGRANGIANA BARREIRA MODIFICADA/PENALIDADE

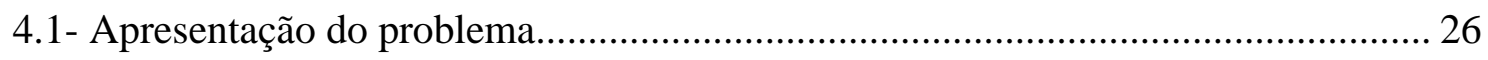

4.2- A função Lagrangiana barreira modificada/penalidade........................................ 27

4.3- O método da função Lagrangiana barreira modificada/penalidade ......................... 31 
4.3.1- O ciclo interno do método da função Lagrangiana barreira modificada/penalidade (iteração de Newton) 32

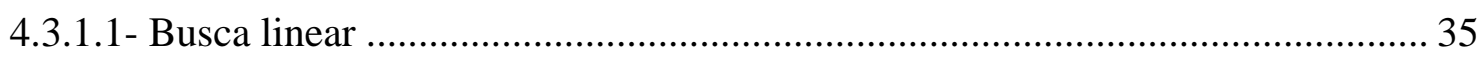

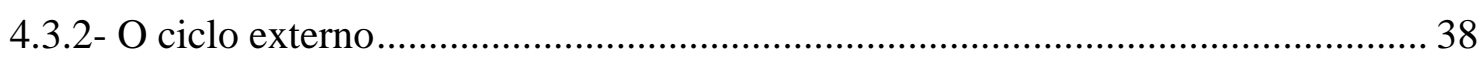

4.3.2.1- Os parâmetros si e o parâmetro

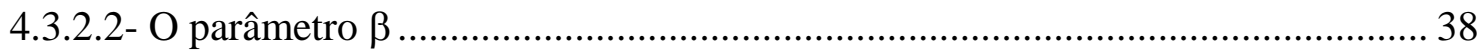

4.3.2.3- Os multiplicadores de Lagrange associados às restrições de desigualdade....... 39

4.3.2.3.1- Inicialização dos multiplicadores de Lagrange.............................................. 41

4.3.2.3.2- Esquema de extrapolação da função barreira clássica ................................... 42

4.3.2.3.3- Método de Newton para a função Lagrangiana barreira clássica/penalidade.45

4.3.2.4- Critérios de parada para o ciclo externo 47

4.3.3- Algoritmo para o método da função Lagrangiana barreira modificada/ penalidade 48

4.3.3.1- Algoritmo da Inicialização com a função Lagrangiana barreira modificada/penalidade 48

4.3.3.2- Algoritmo da função Lagrangiana barreira modificada/penalidade ..... 49

\section{RESULTADOS NUMÉRICOS}

5.1- Exemplo 1 52

5.1.1- Inicialização do método usando a função Lagrangiana barreira clássica/penalidade 53

5.1.2- O método da função Lagrangiana barreira modificada/penalidade ..................... 56

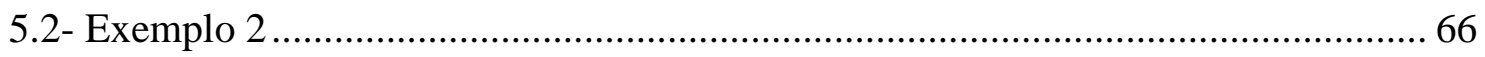

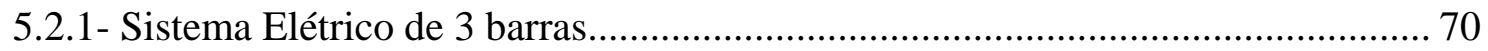

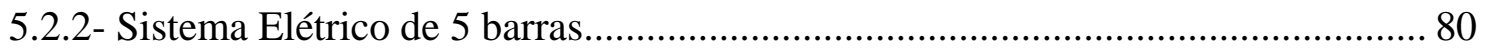

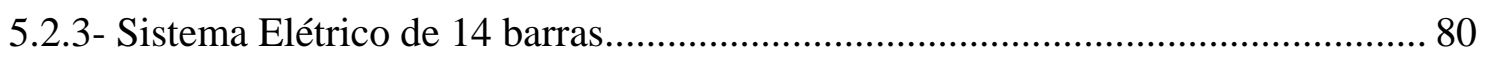

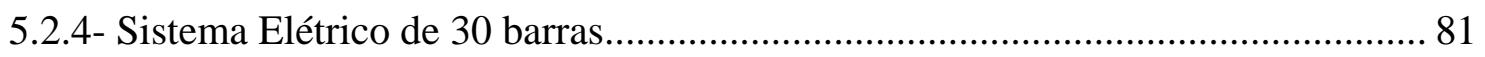

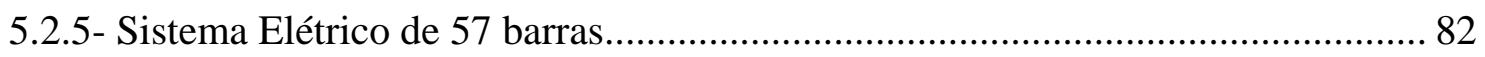

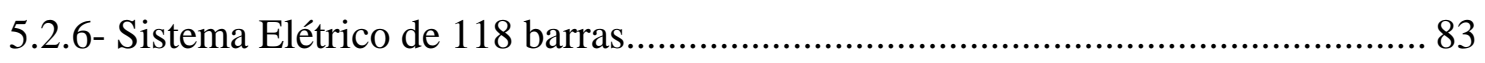

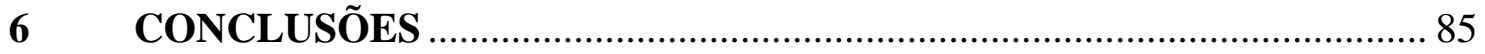

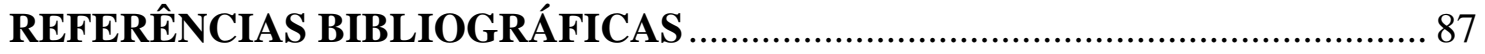


APÊNDICE 1 - ALGORITMOS ESTRUTURADOS

93

APÊNDICE 2 - BANCO DE DADOS DOS SISTEMAS ELÉTRICOS

DE 3 E DE 118 BARRAS 96

APÊNDICE 3 - ESTADO FINAL DOS SISTEMAS ELÉTRICOS

DE 3 E DE 118 BARRAS

102 


\section{LISTA DE FIGURAS}

FIGURA 1 - Ciclo externo do algoritmo do método da FLBMP com inicialização pelo método da FLBCP ............................................................................ 50

FIGURA 2 - Ciclo interno dos métodos da FLBMP e da FLBCP ………………….... 51

FIGURA 3 - Convergência do método da FLBMP referente ao TESTE 1................... 65

FIGURA 4 - Convergência do método da FLBMP referente ao TESTE 2................... 65

FIGURA 5 - Convergência do método da FLBMP referente ao TESTE 3 .................... 66 


\section{LISTA DE TABELAS}

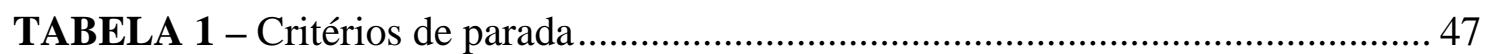

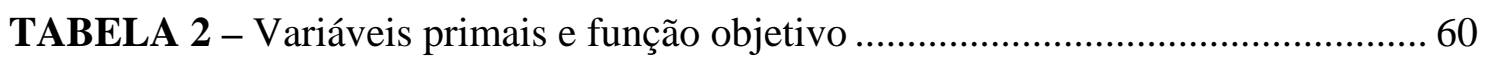

TABELA 3 - Variáveis duais e parâmetro de barreira ............................................... 60

TABELA 4 - Variáveis primais, função objetivo e variáveis duais ............................ 60

TABELA 5 - Estimativas dos multiplicadores de Lagrange e parâmetro de barreira .. 61

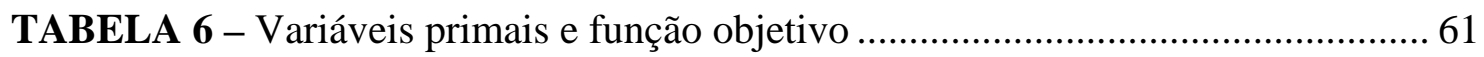

TABELA 7 - Variáveis duais e parâmetro de barreira ................................................... 62

TABELA 8 - Variáveis primais, função objetivo e variáveis duais .............................. 62

TABELA 9 - Estimativas dos multiplicadores de Lagrange e parâmetro de barreira .. 62

TABELA 10 - Variáveis primais e função objetivo .................................................... 63

TABELA 11 - Variáveis duais e parâmetro de barreira .............................................. 63

TABELA 12 - Variáveis primais, função objetivo e variáveis duais ............................. 64

TABELA 13 - Estimativas dos multiplicadores de Lagrange e parâmetro de barreira 64

TABELA 14 - Estado inicial do sistema de 3 barras ....................................................... 75

TABELA 15 - Limites para as tensões e reativos....................................................... 75

TABELA 16 - Valores iniciais das variáveis de folga e dos multiplicadores de

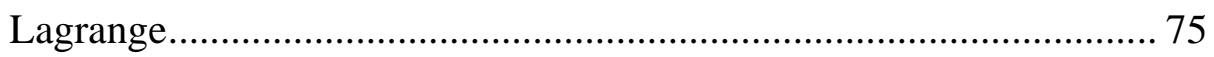

TABELA 17 - Variáveis do sistema de 3 barras na iteração 1 .................................... 76

TABELA 18 - Variáveis de folga e multiplicadores de Lagrange ............................... 76

TABELA 19 - Variáveis do sistema de 3 barras na iteração 2 …................................ 76

TABELA 20 - Variáveis de folga e multiplicadores de Lagrange ............................... 76

TABELA 21 - Variáveis do sistema de 3 barras na iteração 1 ................................... 77

TABELA 22 - Variáveis de folga e multiplicadores de Lagrange ................................ 77

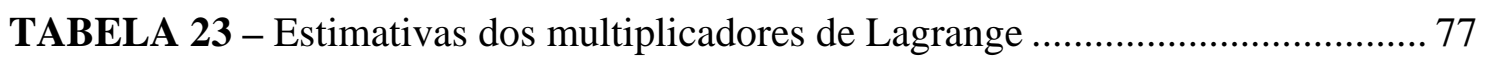

TABELA 24 - Variáveis do sistema de 3 barras na iteração 2 ...................................... 77 
TABELA 25 - Variáveis de folga e multiplicadores de Lagrange ............................... 77

TABELA 26 - Estimativas dos multiplicadores de Lagrange ..................................... 78

TABELA 27 - Variáveis do sistema de 3 barras na iteração 3 ................................... 78

TABELA 28 - Variáveis de folga e multiplicadores de Lagrange ............................... 78

TABELA 29 - Estimativas dos multiplicadores de Lagrange ...................................... 78

TABELA 30 - Variáveis do sistema de 3 barras na iteração 4 ..................................... 79

TABELA 31 - Variáveis de folga e multiplicadores de Lagrange ............................... 79

TABELA 32 - Estimativas dos multiplicadores de Lagrange ..................................... 79

TABELA 33 - Convergência da Função Objetivo no método da FLBCP.................... 79

TABELA 34 - Convergência da Função Objetivo no método da FLBMP................... 79

TABELA 35 - Convergência da Função Objetivo no método da FLBCP..................... 80

TABELA 36 - Convergência da Função Objetivo no método da FLBMP.................... 80

TABELA 37 - Convergência da Função Objetivo no método da FLBCP..................... 81

TABELA 38 - Convergência da Função Objetivo no método da FLBMP.................... 81

TABELA 39 - Convergência da Função Objetivo no método da FLBCP..................... 82

TABELA 40 - Convergência da Função Objetivo no método da FLBMP.................... 82

TABELA 41 - Convergência da Função Objetivo no método da FLBCP..................... 83

TABELA 42 - Convergência da Função Objetivo no método da FLBMP.................... 83

TABELA 43 - Convergência da Função Objetivo no método da FLBCP..................... 84

TABELA 44 - Convergência da Função Objetivo no método da FLBMP.................... 84 


\section{LISTA DE ABREVIATURAS E SIGLAS}

FPO - Fluxo de Potência Ótimo;

FBC - Funções Barreira Clássicas;

FBM - Funções Barreira Modificadas;

FLC - Funções Lagrangianas Clássicas;

FLBMP -Função Lagrangiana Barreira Modificada/Penalidade;

KKT - Karush-Kuhn-Tucker;

MPI - Método de Ponto Interior;

MPE - Método de Ponto Exterior;

COPS - Conjunto de Problemas de Otimização Restrita;

CUTE - Ambiente de Teste Restrito e Irrestrito;

PDBL - Primal-Dual Barreira-Logarítmica;

PPNL - Problema de Programação Não-Linear;

FBMP - Função Barreira Modificada/Penalidade;

FBM - Função Barreira Modificada;

FLBM - Lagrangiana da Função Barreira Modificada;

FLBCP - Função Lagrangiana Barreira Clássica/Penalidade;

FBC - Função Barreira Clássica Logarítmica;

FLBL - Lagrangiana da Função Barreira Clássica Logarítmica;

FBCP - Função Barreira Clássica/Penalidade;

FPM - Função Penalidade Mérito;

PV -Barras de Geração;

NL - Número de Linhas de Transmissão;

NB - Número de Barras do Sistema Elétrico;

NBC - Número de Barras de Carga;

NBCR - Número de Barras de Controle de Reativo; 
NBCCR - Número de Barras de Carga e de Controle de Reativos;

MVA - Mega Volts Ampères;

SL - Barra de Referência (slack);

CR - Barra de Controle de Reativo;

CG - Barra de Carga;

Ang - Ângulo;

Rad - Radianos;

p.u. - Por Unidade;

MW - Mega Watts;

USP - Universidade de São Paulo;

LASEE - Laboratório de Análise de Sistemas de Energia Elétrica;

EESC - Escola de Engenharia Elétrica de São Carlos. 


\section{LISTA DE SÍMBOLOS}

x - variável primal;

$\mathrm{S}_{\mathrm{i}}$ - parâmetro shifit;

$\mu$ - parâmetro de barreira;

$\beta$ - tolerância da aproximação em relação à fronteira da região viável;

$\mathrm{f}(\mathrm{x})$ - função objetivo;

$c(x)$ - restrição de desigualdade;

$\mathrm{m}_{\mathrm{c}}$ - número de restrições de desigualdade $\mathrm{c}(\mathrm{x})$;

$\mathrm{B}(\mathrm{x})$ - termo de barreira;

$\psi(y)$ - função de uma variável y;

$h(x)$ - restrição de igualdade;

$\mathrm{m}_{\mathrm{h}}$ - número de restrições de igualdade $\mathrm{h}(\mathrm{x})$;

z - variável de folga (variável primal);

FL - função Lagrangiana;

$\lambda^{\mathrm{E}}$ - multiplicador de Lagrange associado às restrições de igualdade (variável dual);

$\lambda^{\mathrm{I}}$ - multiplicador de Lagrange associado às restrições de desigualdade (variável dual);

$\nabla_{\mathrm{X}} \mathrm{FL}$ - vetor gradiente da função Lagrangiana;

FLBM - função Lagrangiana barreira modificada

FLBC - função Lagrangiana barreira clássica

H - matriz Hessiana;

S - vetor das direções de busca;

$\mathrm{sx}, \mathrm{sz}, \mathrm{s} \lambda^{\mathrm{E}}$ e $\lambda^{\mathrm{I}}-$ direções de busca para as variáveis $\mathrm{x}, \mathrm{z}, \lambda^{\mathrm{E}}$ e $\lambda^{\mathrm{I}}$;

$\alpha$ - tamanho do passo na direção de busca;

$\alpha_{p}$ - tamanho do passo na direção primal;

$\alpha_{d}$ - tamanho do passo na direção dual;

$\lambda$ - estimativas dos multiplicadores de Lagrange;

r - número de restrições de desigualdade do problema; 
$\tau$ - escalar com valor empírico de 0,9995;

FBM - função barreira modificada;

$\bar{\mu}$ - valor limite para o parâmetro de barreira;

$\mathrm{F}(\mathrm{x}, \lambda, \mu)$ - função barreira de Frisch modificada;

$\mathrm{C}(\mathrm{x}, \lambda, \mu)$ - função barreira de Carrol modificada;

$\Omega_{\mu}$ - conjunto relaxado;

$\rho$ - tamanho do passo encontrado através da regra de Goldstein-Armijo;

$\underline{c}$ - limite inferior da restrição de desigualdade c(x);

$\bar{c}$ - limite superior da restrição de desigualdade c(x);

$\underline{\mathrm{x}}$ - limite inferior da variável canalizada $\mathrm{x}$;

$\overline{\mathrm{x}}$ - limite superior da variável canalizada $\mathrm{x}$;

$\mathrm{m}_{\mathrm{x}}$ - número das variáveis canalizadas $\mathrm{x}$;

$\mathrm{v}^{\mathrm{I}}, \mathrm{v}^{\mathrm{S}}, \xi^{\mathrm{I}}$ e $\xi^{\mathrm{S}}$ - estimativas dos multiplicadores de Lagrange para as restrições de desigualdade, onde S está relacionado com limite superior e I com inferior;

$\phi$ - termo de barreira modificada/penalidade;

$\phi_{\mathrm{FBC}}$ - termo de barreira clássica/penalidade;

Q(x) - função penalidade (extrapolação quadrática);

$\mathrm{q}_{\mathrm{a}}, \mathrm{q}_{\mathrm{b}}$ e $\mathrm{q}_{\mathrm{c}}$ - coeficientes da função penalidade $\mathrm{Q}(\mathrm{x})$ associada à função barreira modificada;

FBMP - função barreira modificada /penalidade;

FLBMP - função Lagrangiana barreira modificada/penalidade;

$\mathrm{G}$ - vetor gradiente;

$\mathrm{G}_{\mathrm{FLBMP}}$ - vetor gradiente da FLBMP;

$\mathrm{H}_{\mathrm{FLBMP}}-$ matriz Hessiana da FLBMP;

$\mathrm{X}$ - vetor das variáveis primais e duais;

p - indexador das iterações internas;

$\mathrm{T}$ - transposição de matriz ou vetor;

w - constante suave;

$\alpha_{0}$ - passo inicial;

BL - indexador das iterações da busca linear;

FPM - função penalidade mérito;

$\mathrm{M}$ - termo de penalidade; 
$\varepsilon$ - precisão;

L - indexador das iterações externas;

$\gamma$ - parâmetro de redução do parâmetro de barreira;

$\mu_{0}$ - parâmetro de barreira inicial;

$\mathrm{u}_{1}, \mathrm{u}_{2}, \mathrm{u}_{3}-$ critérios de parada;

$\eta$ - precisão para os critérios de parada;

FBC - função barreira clássica;

FLBCP - função Lagrangiana barreira clássica/penalidade;

$\mathrm{G}_{\mathrm{FLBCP}}$ - vetor gradiente da FLBCP;

$\mathrm{H}_{\mathrm{FLBCP}}$ - matriz Hessiana da FLBCP;

a, b e c - coeficientes da função penalidade Q(x) associada à função barreira clássica.

$\mathrm{g}_{\mathrm{i}}$-condutância da linha i conectada entre a barra k e m;

k e m - barras do sistema;

$\mathrm{V}_{\mathrm{k}}$ e $\mathrm{V}_{\mathrm{m}}$ - magnitudes de tensão nas barras k e m, respectivamente;

$\theta_{\mathrm{km}}$ - ângulo da tensão entre as barras k e m, respectivamente;

$\mathrm{y}_{\mathrm{km}}$ - admitância da linha entre as barras k e m;

$\mathrm{P}^{\mathrm{G}}$ e $\mathrm{P}^{\mathrm{C}}$ - potências ativas, gerada e consumida, respectivamente;

$\mathrm{Q}^{\mathrm{G}}$ e $\mathrm{Q}^{\mathrm{C}}$ - potências reativas, gerada e consumida, respectivamente;

$\mathrm{K}$ - conjunto de todas as barras vizinhas à barra $\mathrm{k}$, incluindo ela mesma.

$\underline{Q}$ e $\overline{\mathrm{Q}}$ - limites mínimos e máximos de geração de potência reativa, respectivamente;

$\underline{\mathrm{V}}$ e $\overline{\mathrm{V}}$ - limites mínimos e máximos da magnitude de tensão, respectivamente;

NL - número de linhas de transmissão;

NB - número de barras do sistema elétrico;

NBC - número de barras de carga;

NBCR - número de barras de controle de reativo;

NBCCR - número de barras de carga e de controle de reativos. 


\section{CAPÍTULO 1}

\section{INTRODUÇÃO}

Problemas de otimização não-linear são encontrados nas mais diversas áreas do conhecimento, como engenharia, química, agronomia, medicina, entre outras. Esses problemas, em geral, nem sempre possuem uma resolução fácil, devido à sua nãolinearidade e quantidade de variáveis, necessitando de métodos numéricos eficientes para alcançar uma convergência satisfatória na determinação da sua solução. Dentre estes métodos, temos os métodos de barreira que transformam um problema restrito em um problema irrestrito e introduzem as restrições na função objetivo através de um parâmetro de barreira, que impede a aproximação de um ponto factível à fronteira da região factível.

É utilizada neste trabalho para resolver problemas de otimização restrita nãolineares, a teoria dos métodos da função barreira modificada, que foi desenvolvida por Polyak, em 1992. Tais métodos combinam as melhores propriedades da função barreira clássica e função Lagrangiana clássica.

Comparadas com as funções barreira clássicas (FBC), as funções barreira modificadas (FBM) e suas derivadas são definidas na solução, não crescem para o infinito, sua matriz Hessiana da função Lagrangiana não se torna mal condicionada e o parâmetro de barreira não necessita tender para zero durante o processo de 
convergência. A qualidade mais importante da FBM é a representação explicita da estimativa dos multiplicadores de Lagrange, pois estes auxiliam no processo de convergência do método.

Segundo Polyak (1992), em contraste com as funções Lagrangianas clássicas (FLC), a FBM é convexa na vizinhança da solução para problemas de programação não convexos, desde que as condições de otimalidade de segunda ordem estejam satisfeitas. Com a estimativa ótima dos multiplicadores de Lagrange, o extremo irrestrito da FBM existe e coincide com a solução do problema inicial. As funções duais, às quais são baseadas as FBM, são tão suaves quanto às funções iniciais do problema primal. O problema dual é sempre convexo, independente de o problema primal ser ou não convexo, e tem importantes propriedades locais próximo à solução.

O método proposto, neste trabalho, denominado de método da função Lagrangiana barreira modificada/penalidade, é desenvolvido para resolver problemas não-lineares restritos onde todas as restrições de desigualdade são supostas do tipo “ $\geq$ ”; as variáveis canalizadas são desmembradas em duas restrições de desigualdade, e as restrições canalizadas são separadas em duas restrições de desigualdade e uma de igualdade, através do acréscimo de variáveis de folga. Todas as restrições de desigualdade são relaxadas e tratadas através da função barreira modificada/penalidade, dando origem a um problema equivalente. Associa-se a esse problema uma função Lagrangiana denominada função Lagrangiana barreira modificada/penalidade (FLBMP). As condições necessárias de primeira ordem são aplicadas à FLBMP, gerando um sistema de equações não-lineares, o qual é resolvido pelo método de Newton. Esse processo de linearização gera um sistema de equações cuja solução nos fornece as direções de busca para atualização das variáveis primais e duais e o passo é determinado por um procedimento de busca linear. Os multiplicadores de Lagrange são atualizados através de um esquema proposto por BREITFELD \& SHANNO (1994b). Com o objetivo de verificar a eficiência do método, aplicamos em um problema teste e em problemas de FPO. Um esquema para inicialização dos multiplicadores de Lagrange é utilizado. 
Este trabalho está organizado como segue:

No capítulo 2, apresentamos um histórico sobre a função barreira.

No capítulo 3, expomos os métodos de função barreira clássica e modificada.

No capítulo 4, desenvolvemos o método função Lagrangiana barreira modificada/penalidade.

No capítulo 5, os resultados numéricos do método proposto para um exemplo teórico e para problemas de FPO são apresentados.

E finalmente, no capítulo 6, descrevemos as conclusões obtidas dos resultados da aplicação do método e as perspectivas de continuidade deste trabalho. 


\section{CAPÍTULO 2}

\section{HISTÓRICO}

Neste capítulo apresentamos um levantamento bibliográfico de trabalhos que utilizam a função barreira e suas variantes. Nosso objetivo é fornecer um posicionamento histórico para a abordagem proposta neste trabalho.

O método da função barreira, ou método de barreira, é utilizado para a resolução de problemas de otimização com restrições de desigualdade, cujo interior é não vazio. Pode ser visto como um caso particular do método de penalidade, mas diferencia-se deste por exigir uma barreira interna, ou seja, por trabalhar no interior da região factível, utilizando uma função auxiliar que cresce indefinidamente próxima à fronteira e uma seqüência decrescente de fatores de barreira.

A função barreira logarítmica foi estudada por Frisch (1955) para problemas de programação convexa. Outra função barreira, denominada função barreira inversa foi proposta por Carrol (1961), sob o nome de Created Response Surface Technique. O método de barreira foi realmente popularizado por Fiacco e McCormick (1968), que realizaram um estudo teórico mais detalhado do método e desenvolveram um novo; juntando a função barreira e a função penalidade em uma mesma função auxiliar. Uma versão revisada desse trabalho pode ser encontrada em Fiacco e McCormick (1990). 
Murray (1971) apresentou um estudo sobre expressões analíticas para autovetores e autovalores de funções barreira em um mínimo intermediário; justificou que o objetivo desse desenvolvimento é a análise do comportamento da função na “vizinhança” do ponto ótimo. Também citou que, na resolução de métodos da função barreira, as técnicas de Newton e quasi-Newton são as mais utilizadas, e que necessitam de uma estimativa da inversa da Hessiana, a qual pode ser determinada analiticamente por esse estudo.

Os pesquisadores que trabalharam com o método da função barreira verificaram que ele apresenta alguns problemas, tais como: o mal condicionamento da matriz Hessiana da função Lagrangiana quando seu fator de barreira tende a zero; a dificuldade na escolha do fator de barreira e na escolha de uma solução inicial; a não-existência da derivada na solução e o aumento ilimitado da função barreira na vizinhança da fronteira. Em virtude desses problemas, na década de 70, o entusiasmo no uso da função barreira diminuiu sensivelmente.

O interesse pelo método da função barreira reapareceu somente após a apresentação feita por Karmarkar, em 1984, de seu método projetivo para programação linear, cujo maior mérito, como citado em Gonzaga (1989), foi o de ter mostrado que o problema de programação linear é, de fato, um caso particular da programação nãolinear e é tratável por técnicas da mesma área. Seu método dependia da utilização de uma transformação não-linear conhecida como Transformação Projetiva, e seu objetivo era “caminhar” pelo interior da região factível. O sucesso de tal método deu-se por dois motivos: à sua complexidade polinomial (em comparação com a complexidade exponencial do método simplex) e, ao seu sucesso computacional para problemas de grande porte. Esse método também ficou conhecido como método de pontos interiores.

Após Karmarkar ter proposto o método de pontos interiores para programação linear foram apresentados na literatura especializada vários trabalhos com variações do seu algoritmo original, como pode ser visto em Gonzaga (1989), Boukari e Fiacco (1995), Forsgren et al. (2002), entre outros. Uma das variantes do método projetivo de Karmarkar é o método afim-escala, que utiliza uma transformação afim em detrimento à transformação projetiva. O método afim-escala possui duas variantes: o afim-escala 
primal, para solucionar problemas lineares na forma padrão, e o afim-escala dual, para solucionar problemas lineares na forma de desigualdades. Como outras variantes podem ser citados os métodos primais de trajetória central e os primais-duais ou pathfollowing, estes últimos assim como o método afim-escala e suas variantes podem ser encontrados em Matumoto (1996).

Gill et al. (1986) utilizaram o método de Karmarkar para desenvolver o método da barreira de Newton projetada para solução de problemas lineares de otimização; apresentaram uma descrição completa do novo método, e também mostraram que para determinados tipos de problemas de programação linear e para uma dada escolha do fator de barreira e do tamanho do passo, o algoritmo deles é equivalente ao de Karmarkar.

Diversos autores foram responsáveis pelo desenvolvimento dos métodos de pontos interiores, na década de 80 e inicio da década 90, entre eles pode-se citar Mehrotra (1992) e outros autores que podem ser encontrados nos trabalhos de Gonzaga (1989) e de Boukari e Fiacco (1995). Em virtude do interesse despertado por Karmarkar e seus seguidores na década de 80, a função barreira logarítmica passou novamente a ser usada como uma ferramenta alternativa de trabalho, surgindo novos tipos de função barreira.

Polyak (1992) desenvolveu uma teoria de métodos de barreira modificada para resolver problemas de otimização restrita. Tais métodos combinam as melhores propriedades da função Lagrangiana clássica e da função barreira clássica, evitando os problemas que ambas enfrentam. Por exemplo, em contraste com a função barreira clássica, as funções barreira modificadas são definidas na solução; estas são suaves na vizinhança do ótimo e não vão para infinito quando o ótimo se aproxima. Já em contraste com a Lagrangiana clássica, é convexa na vizinhança da solução para problemas de programação não convexos, se as condições de segunda ordem são satisfeitas. As funções duais, nas quais são baseadas as funções barreira modificadas, são tão suaves quanto às funções do problema primal. O problema dual é sempre convexo, independentemente de o problema primal ser ou não convexo, e tem importantes propriedades locais próximas à solução. Segundo Polyak, a finalidade que o 
método de barreira modificada tem para os métodos de pontos interiores é a mesma que o método da função Lagrangiana aumentada tem para os métodos de penalidade, isto é, ajudá-lo a "driblar” suas dificuldades. Por esse motivo, o autor considera a função barreira modificada como uma Lagrangiana aumentada interior. Apresenta diferentes versões do método para o problema de programação convexa e não convexa. Tais versões consistem no trabalho com um parâmetro de barreira fixo, na alteração desse parâmetro de barreira em um determinado nível e na alteração contínua, fornecendo convergência linear e super linear.

Wright (1994), em seu trabalho, cita Murray (1971), o qual apresentou que as matrizes Hessianas da função barreira logarítmica ficam mal condicionadas nos pontos sobre a trajetória de convergência quando o processo se aproxima da solução. Wright dedicou seu trabalho à exploração do comportamento da matriz Hessiana associada ao problema de barreira. Mostrou o fato de a Hessiana da função barreira ser mal condicionada na região próxima à solução, a não ser no caso de o número de restrições ativas serem nulas ou iguais ao número de variáveis. Também discutiu uma fatorização de Cholesky para a matriz com posto deficiente.

Encontra-se em Boukari e Fiacco (1995), um levantamento cronológico, para o período de 1969 a 1993, de trabalhos que têm por objetivo melhorar o método da função barreira e um histórico a respeito dos métodos de pontos interiores.

Wright (1995) estudou a aplicação do método de Newton ao método da função barreira, ressaltando o fato de esta ser problemática em razão do mal condicionamento da matriz Hessiana da função Lagrangiana; classificou como passo puro de Newton, o passo igual a 1. Definiu métodos de passo curto, nos quais são exigidas poucas minimizações (ou apenas uma) do fator de barreira, e métodos de passo longo, nos quais são exigidas várias minimizações do fator de barreira. O principal resultado desse estudo de Wright foi a demonstração de que um passo puro de Newton, em um método de passo longo, pode não ter sucesso.

El-Bakry et al. (1996) tiveram como objetivo principal apresentar uma formulação factível de métodos de pontos interiores para problemas de programação 
não-linear, a partir da formulação já existente para programação linear. Para atingir esse objetivo realizaram um estudo de um método de pontos interiores, para o caso linear, caracterizando as condições de Karush-Kuhn-Tucker (KKT) perturbadas. Mostraram que a trajetória do método de Newton para a resolução das condições de KKT perturbadas associadas a um problema de programação linear e a trajetória do mesmo método, e para a resolução das condições de KKT para o problema da função barreira logarítmica, não coincidem, mas determinam a mesma solução para o problema de programação linear. Concluíram que as condições perturbadas de KKT não são as condições de KKT para o problema da função barreira logarítmica. Os autores compararam o uso das condições de KKT perturbadas no método da função barreira logarítmica ao uso do método dos multiplicadores no método da função penalidade. Tanto as condições de KKT perturbadas quanto o método dos multiplicadores são utilizados para melhorar o condicionamento da matriz Hessiana da função Lagrangiana. Apresentaram uma formulação para programação não-linear denominada método de ponto interior primal-dual Newton, propriedades de convergência local e global e alguns experimentos computacionais.

Em 1996, Breitfeld e Shanno baseados no trabalho de Polyak (1992), apresentaram o método de barreira-penalidade para problemas de programação nãolinear. Tiveram como objetivo o desenvolvimento, a partir dos métodos de função barreira logarítmica modificada, de um novo método, no qual os termos logarítmicos são extrapolados por aproximações quadráticas. Também foi apresentada, por eles, uma implementação detalhada desse método, incluindo a formulação da nova função, o valor inicial das variáveis e o critério de convergência. Para a otimização irrestrita, os autores apresentaram um método de Newton modificado, em que é usada uma Hessiana modificada, para uma busca linear, e os critérios de convergência do método. Breitfeld e Shanno, nesse estudo, destacam que os resultados computacionais são promissores.

Melman (1996) propôs um procedimento de busca linear em métodos de função barreira para problemas de programação quadrática, com restrições quadráticas e convexas. Apresentou a aplicação desse procedimento de busca linear aos seguintes métodos de pontos interiores: método de trajetória central, método da função barreira de Carrol (1961) e método da função barreira modificada, vista em Polyak (1992). 
Foi apresentado por Conn et al. (1997) uma classe de métodos denominados métodos de barreira Lagrangianos. Esses métodos usam a mesma função barreira modificada estudada em Polyak (1992). Conn et al. utilizaram uma função barreira Lagrangiana baseados nos seguintes motivos: funções barreira determinam restrições ativas na solução de um modo mais eficiente que os métodos do conjunto ativo, sendo isto válido, também, para a função barreira Lagrangiana; os métodos de pontos interiores para programação não-linear são menos sensíveis à degeneração que os métodos de restrições ativas. Experimentos numéricos indicam que esse método é superior ao da barreira clássica, evita o mal condicionamento que ocorre neste, impedindo dificuldades numéricas. Os métodos de barreira Lagrangianos são usados na resolução de problemas de grande porte, com a vantagem de evitar variáveis de folga. Os autores forneceram um algoritmo, o qual converge para um ponto em que as condições de KKT são satisfeitas. Finalmente, destacaram que seu método de barreira Lagrangiano resolveu noventa por cento dos problemas-teste.

Em 1998, Vassiliadis e Brooks, baseados no trabalho de Polyak (1992) e Breitfeld e Shanno (1996) apresentaram o método de barreira-penalidade para problemas de programação quadrática de grande porte. Também basearam o método na função barreira modificada e na extrapolação quadrática dos termos de barreira. Apresentaram a atualização dos parâmetros de barreira-penalidade, um algoritmo para o método e a inicialização dos multiplicadores de Lagrange via o método da função barreira logarítmica, que, além de conseguir uma aproximação, obtém-se também uma solução inicial melhorada.

Wright e Jarre (1999) apresentaram o método de barreira logarítmica - Newton para a resolução de problemas não-lineares com restrições de desigualdade. Os autores mostraram que, para a função objetivo linear, um passo efetivo pode ser tomado na direção de Newton, depois de cada redução no fator de barreira, obtendo um bom comportamento do método próximo à solução. Isso contrasta com o caso da função objetivo não-linear, em que o método de Newton pode falhar quando o fator de barreira vai para zero. Forneceram o algoritmo em que o método da função barreira logarítmica utiliza o método de Newton clássico, empregando a regra de Armijo de busca linear. Por 
fim aplicaram esse algoritmo a um exemplo numérico para verificar seu comportamento.

Ainda em 1999, Shanno e Vanderbei mostraram um algoritmo de ponto interior para a programação não-linear não convexa que faz uma perturbação na matriz Hessiana da função Lagrangiana caso esta não seja definida positiva.

Shanno e Vanderbei (2000) desenvolveram uma extensão do algoritmo de ponto interior proposto pelos mesmos em 1999. Esse algoritmo também realizava uma perturbação na matriz Hessiana da função Lagrangiana caso esta não seja definida positiva. Os autores apresentaram os métodos primal versus dual e de alta ordem que tentam usar cada fatoração da matriz Hessiana da função Lagrangiana mais de uma vez para melhorar a eficiência computacional. Os resultados mostraram que diferentemente da programação quadrática convexa e da linear as correções para a trajetória central não são úteis para programação não-linear não convexa. Mas que uma variante do algoritmo preditor-corretor de Mehrotra (1992) definitivamente pode melhorar o desempenho do método. Os autores desenvolveram também, uma estratégia dual para determinar se aplicar o método preditor-corretor várias vezes é, ou não, mais vantajoso que o procedimento padrão.

Forsgren et al. (2002) realizaram uma revisão e uma pesquisa recente sobre métodos de pontos interiores para otimização restrita não-linear. Começam pelo surgimento dos métodos de pontos interiores com o trabalho de Karmarkar e mostram todo o seu desenvolvimento e progresso. Os pesquisadores revisaram os conceitos de otimização, métodos de barreira e suas variações e o método de barreira clássica Newton. Destacam a bem sucedida trajetória dos métodos de pontos interiores com a função barreira logarítmica na otimização restrita.

Bakhtiari e Tits (2003) propuseram e analisaram um método de ponto interior primal-dual do tipo factível para programação não-linear, com a propriedade adicional monotonicamente descendente, a qual diminui a função objetivo a cada iteração. Uma característica deste método é o uso de valores diferentes no vetor do parâmetro de barreira para cada restrição, com o propósito de melhorar a direção de busca construída 
o que evita pontos estacionários que não satisfazem as condições de KKT. Segundo os autores, os recursos do esquema proposto incluem simplicidade relativa do algoritmo e da análise de convergência, propriedades de convergência locais e globais e o bom desempenho em testes preliminares. Além disso, o algoritmo não exige um ponto inicial interior, este ponto pode iniciar no limite do conjunto factível.

Byrd et al. (2003) apresentaram um método de ponto interior factível usando variáveis auxiliares (folga ou excesso) que se origina de uma modificação de métodos infactíveis para otimização não-linear. Descreveram uma base para transformação de métodos infactíveis, usando variável auxiliar, em métodos factíveis. Nessa base, algoritmos de pontos interiores factíveis e infactíveis podem ser considerados como variantes do mesmo método básico. A factibilidade é controlada por um reajuste ou não das variáveis auxiliares após a execução de um passo teste, e pela forma como essas variáveis são reajustadas. Usando essa flexibilidade pode-se escolher forçar a factibilidade em relação a algumas, todas, ou nenhuma das restrições de desigualdade dependendo do que é necessário ou esperado. Segundo os autores, a estratégia do reajuste da variável auxiliar pode experimentar dificuldades nos problemas com restrições de igualdade e desigualdade.

Chen e Vassiliadis (2003) propuseram um método que se baseia na função barreira modificada, na função Lagrangiana, no método de Newton, nas extrapolações quadráticas. Aplicaram o método em alguns exemplos teóricos e em alguns problemas da área de Química.

Gonzaga e Cardia (2004) listaram várias propriedades úteis dos algoritmos de pontos centrais para problemas de programação linear e apresentaram um estudo da função barreira logarítmica, do centro analítico e da trajetória central. Mostraram que a variação da função barreira primal ao longo da trajetória central depende da proximidade para o centro analítico. Estudaram o algoritmo de path-following de passo curto e determinaram qual o maior tamanho que pode ser atribuído aos passos curtos. Mostraram que a variação da função barreira em cada iteração do algoritmo de passo curto tem um limite inferior que não depende do problema. Estabeleceram uma relação 
entre passos de Newton primal-dual e primal nos métodos de path-following, e propuseram um algoritmo primal preditor-corretor.

Griva (2004) apresentou um algoritmo para resolver problemas de programação não-linear. O algoritmo está baseado na combinação de métodos de ponto interior e exterior. Este último também é conhecido como o método primal-dual não-linear rescaling. O autor mostrou que em certos casos quando o método de ponto interior (MPI) não alcança a solução com um alto nível de precisão, o uso do método de ponto exterior (MPE) pode “revolver” esta situação. O resultado é demonstrado resolvendo problemas do COPS (Conjunto de Problemas de Otimização Restrita) e um conjunto de problemas CUTE (Ambiente de Teste Irrestrito e Restrito) usando o aplicativo para programação não linear solver LOQO o qual foi modificado para incluir a sub-rotina do método de ponto exterior.

Akrotirianakis e Rustem (2005) apresentaram um algoritmo de pontos interiores primal-dual para resolver problemas de programação não-linear e restritos. As restrições de desigualdade são acrescidas na função objetivo por meio da função barreira logarítmica e as restrições de igualdade são tratadas usando uma função penalidade quadrática adaptativa. O parâmetro de penalidade é determinado usando uma estratégia que assegura uma propriedade descendente para uma função mérito. A convergência global do algoritmo é alcançada por meio da redução monotônica de uma função mérito. Segundo os autores, os resultados computacionais mostraram que o algoritmo pode resolver problemas de grande porte e complexos de forma eficiente e robusta.

Sousa et al. (2006) mostraram um método de resolução de problemas nãolineares e não convexos baseados na metodologia de pontos interiores. Neste método, as condições de otimalidade de primeira ordem são aplicadas à função Lagrangiana barreira modificada resultando num sistema não-linear, cuja solução é determinada através da utilização do método de Newton. Uma das vantagens deste método é que o fator de barreira não tende ao infinito quando o ótimo se aproxima. Seu potencial foi mostrado na resolução de problemas de fluxo de potência ótimo (FPO). O número de iterações do método está diretamente ligado à escolha dos fatores iniciais de barreira e seus respectivos parâmetros de correção. O método exige uma experiência prévia do 
sistema que será resolvido para um adequado ajuste do fator de barreira e de seu parâmetro de barreira.

Finalmente, Kocvara e Stingl (2007) propuseram um algoritmo baseado na função barreira modificada e que utiliza “solvers” iterativos para calcular as direções de busca para problemas de programação linear semi-definidos de grande porte. A idéia do uso destes “solvers” é evitar o cálculo explícito da Matriz de Newton ou por um esquema implícito no produto matriz-vetor ou por alguma fórmula de diferenças finitas. Tudo isso leva a um enorme ganho de memória computacional para o algoritmo e, além disso, para alguns problemas, a aceleração do algoritmo. 


\section{CAPÍTULO 3}

\section{MÉTODOS DA FUNÇÃO BARREIRA E DA FUNÇÃO BARREIRA MODIFICADA}

Neste capítulo apresentamos o método primal-dual barreira logarítmica e o método da barreira modificada, os quais fornecerão suporte teórico para a apresentação da abordagem proposta.

\subsection{O MÉTODO PRIMAL-DUAL BARREIRA LOGARÍTMICA}

O método da função barreira, ou método de barreira, é utilizado para a resolução de problemas com restrições de desigualdade, cujo interior é não vazio. Pode ser visto como um caso particular do método de penalidade, mas diferencia-se deste por exigir uma barreira interna, ou seja, por trabalhar no interior da região factível, utilizando uma função auxiliar que cresce indefinidamente próxima à fronteira e uma seqüência decrescente de fatores de barreira.

A função barreira logarítmica foi estudada por Frisch (1955) para problemas de programação convexa. Outra função barreira, denominada função barreira inversa foi proposta por Carrol (1961). O método de barreira foi realmente popularizado por Fiacco 
e McCormick (1968), que realizaram um estudo teórico mais detalhado do método e desenvolveram um novo; juntando a função barreira e a função penalidade em uma mesma função auxiliar.

Os métodos de barreira transformam o problema restrito em um problema irrestrito e introduzem as restrições na função objetivo através de um parâmetro de barreira, que impede a aproximação de um ponto factível à fronteira da região factível. Trabalhando no interior dessa região, tais parâmetros geram barreiras que impedem as variáveis de violarem seus limites. Logo, parte-se de um ponto factível e geram-se novos pontos factíveis. Uma das vantagens desse método é a obtenção de, pelo menos, uma solução factível, caso ocorra uma parada prematura do mesmo, pois esse método trabalha somente com problemas de desigualdade cujo interior é não-vazio. Assim, assume-se o problema (3.1) somente com restrições de desigualdade, da seguinte forma:

$$
\begin{array}{ll}
\text { Minimizar } & f(x) \\
\text { sujeito a : } & c_{i}(x) \geq 0, \quad i=1,2, \ldots, m_{c},
\end{array}
$$

em que $x \in \mathrm{R}^{\mathrm{n}}$.

Com o objetivo de garantir a permanência no interior da região factível, pode-se gerar o seguinte problema de barreira:

Minimizar $\{f(x)+\mu B(x): c(x)>0\}$,

$\mathrm{X}$

em que $\mu>0$ é denominado parâmetro de barreira, e $B(x)$ é uma função barreira nãonegativa e contínua no interior da região factível $\{\mathrm{x} ; \mathrm{c}(\mathrm{x})>0\}$ e tende ao infinito à medida que a solução se aproxima da fronteira, a partir do interior. Define-se, então:

$$
\mathrm{B}(\mathrm{x})=\sum_{\mathrm{i}=1}^{\mathrm{m}_{\mathrm{c}}} \psi\left[\mathrm{c}_{\mathrm{i}}(\mathrm{x})\right]
$$


em que $\psi$ é uma função de uma variável y, contínua sobre $\{y ; y>0\}$, e satisfaz

$$
\psi(y)>0 \text {, se } y>0 \quad \text { e } \lim _{\mathrm{y} \rightarrow 0^{+}} \psi(y)=\infty \text {. }
$$

A função $\mathrm{f}(\mathrm{x})+\mu \mathrm{B}(\mathrm{x})$ é denominada função auxiliar; a função barreira pode assumir várias formas, como:

$$
\begin{aligned}
& \mathrm{B}(\mathrm{x})=\sum_{\mathrm{i}=1}^{\mathrm{m}_{\mathrm{c}}} \frac{1}{\mathrm{C}_{\mathrm{i}}(\mathrm{x})} ; \\
& \mathrm{B}(\mathrm{x})=-\sum_{\mathrm{i}=1}^{\mathrm{m}_{\mathrm{c}}} \ln \left[\mathrm{c}_{\mathrm{i}}(\mathrm{x})\right] .
\end{aligned}
$$

A função (3.5) é denominada barreira clássica ou inversa e foi estudada por Carrol (1961) e (3.6) é denominada função barreira logarítmica e foi estudada por Frisch (1955).

Quando $\mu \rightarrow 0$ e $\mathrm{B}(\mathrm{x}) \rightarrow \infty$, tem-se que $\mu \mathrm{B}(\mathrm{x})$ se aproxima da função barreira ideal, descrita em (3.2), e a solução do problema de barreira converge para a solução do problema (3.1).

Observa-se que (3.2) é um problema irrestrito e pode ser tão complexo quanto (3.1), pois é exigida uma solução inicial interior à região factível. O método trabalha com pontos interiores a essa região, ao ponderar os pontos que se aproximam da fronteira impede que estes saiam da região factível e a restrição pode ser ignorada. Temse, realmente, um problema irrestrito, para o qual poderá ser utilizada uma técnica de otimização irrestrita.

A seguir apresentamos o método primal-dual barreira-logarítmica (PDBL). A fundamentação teórica para métodos de pontos interiores consiste na construção de três blocos cruciais: o método de Newton para resolver equações não-lineares e conseqüentemente para a otimização irrestrita, o método dos multiplicadores de Lagrange para a otimização com restrições de igualdade, o método de barreira de Fiacco e McCormick (1968) para a otimização com restrições de desigualdade. Entre as 
variantes de métodos de pontos interiores, o método PDBL é o mais utilizado devido a sua eficiência e facilidade de implementação.

A resolução de um problema do tipo:

$$
\begin{aligned}
& \operatorname{Minimizar} f(x) \\
& \text { sujeito a: } h_{j}(x)=0, j=1, \ldots, m_{h} \\
& \qquad c_{i}(x) \geq 0, i=1, \ldots, m_{c}
\end{aligned}
$$

pelo método PDBL exige que as restrições de desigualdades sejam transformadas em igualdades por meio da introdução de variáveis de folga ou excesso positivas. Portanto, o problema (3.7) modificado pode ser apresentado como:

$$
\begin{array}{ll}
\operatorname{Minimizar} & \mathrm{f}(\mathrm{x}) \\
\text { sujeito a : } & \mathrm{h}_{\mathrm{j}}(\mathrm{x})=0, \mathrm{j}=1, \ldots, \mathrm{m}_{\mathrm{h}} \\
& \mathrm{c}_{\mathrm{i}}(\mathrm{x})-\mathrm{z}_{\mathrm{i}}=0, \mathrm{i}=1, \ldots, \mathrm{m}_{\mathrm{c}} \\
& \mathrm{z}_{\mathrm{i}} \geq 0
\end{array}
$$

$\operatorname{com} \mathrm{z}_{\mathrm{i}} \in \mathrm{R}^{\mathrm{m}_{\mathrm{c}}}$ uma variável de excesso.

Adiciona-se uma função barreira logarítmica à função objetivo de forma a garantir a não negatividade dessa variável de excesso:

$$
\begin{aligned}
& \operatorname{Minimizar} f(x)-\mu \sum_{i=1}^{m_{c}} \ln \left(z_{i}\right) \\
& \text { sujeito a : } h_{j}(x)=0, j=1, \ldots, m_{h} \\
& \qquad c_{i}(x)-z_{i}=0, i=1, \ldots, m_{c}
\end{aligned}
$$

em que $\mu$ é denominado parâmetro de barreira.

A variável $z_{i}, i=1, \ldots, m_{c}$, é estritamente positiva e o parâmetro de barreira $\mu$ é um número positivo que tende a zero. Quando isso acontece, a solução do subproblema 
apresentado em (3.9), $x(\mu)$, aproxima-se de $x^{*}$ a solução do problema (3.1). A função Lagrangiana associada ao subproblema (3.9) é:

$$
F L=f(x)-\mu \sum_{i=1}^{m c} \ln \left(z_{i}\right)-\sum_{j=1}^{m h} \lambda_{j}^{E} h_{j}(x)-\sum_{i=1}^{m c} \lambda_{i}^{I}\left[c_{i}(x)-z_{i}\right]
$$

em que:

$\lambda_{\mathrm{j}}^{\mathrm{E}}, \mathrm{j}=1, \ldots, \mathrm{m}_{\mathrm{h}}$ e $\lambda_{\mathrm{i}}^{\mathrm{I}}, \mathrm{i}=1, \ldots, \mathrm{m}_{\mathrm{c}}$, são os vetores dos multiplicadores de Lagrange, denominados de variáveis duais.

As condições necessárias de primeira-ordem são aplicadas em (3.10), gerando:

$$
\nabla_{\mathrm{X}} \mathrm{FL}=0
$$

com: $\mathrm{X}=\left(\mathrm{x}, \mathrm{z}, \lambda^{\mathrm{E}}, \lambda^{\mathrm{I}}\right)^{\mathrm{T}}$.

A equação (3.11) representa um sistema de equações não-lineares, o qual é resolvido pelo método de Newton, que gera um sistema do tipo $\mathrm{HS}=-\nabla_{\mathrm{X}} \mathrm{FL}$; em que H é a matriz Hessiana da função Lagrangiana; $\nabla_{X}$ FL é o vetor gradiente e o vetor direção de busca $S=\left(s x, s z, s \lambda^{E}, s \lambda^{I}\right)^{T}$ é utilizado para atualizar as variáveis $x, z, \lambda^{\mathrm{E}}$ e $\lambda^{\mathrm{I}}$ como segue:

$$
\begin{aligned}
& x^{k+1}=x^{k}+\alpha s x \\
& z^{k+1}=z^{k}+\alpha s z \\
& \left(\lambda^{E}\right)^{k+1}=\left(\lambda^{E}\right)^{k}+\alpha s \Delta \lambda^{E} \\
& \left(\lambda^{I}\right)^{k+1}=\left(\lambda^{I}\right)^{k}+\alpha s \Delta \lambda^{I}
\end{aligned}
$$

em que o tamanho de passo $\alpha \in(0,1]$ é escolhido para preservar a positividade do vetor $\mathrm{z}$ e o sinal do vetor $\lambda^{\mathrm{I}}$. Isto se traduz por:

$$
\alpha_{\mathrm{p}}^{\max }=\min \left\{\frac{\mathrm{z}}{|\mathrm{sz}|}: \mathrm{sz}<0\right\}
$$




$$
\begin{aligned}
& \alpha_{d}^{\max }=\min \left\{\frac{\lambda^{I}}{\left|s \lambda^{I}\right|}: s \lambda^{I}<0\right\} \\
& \alpha=\min \left\{\tau \alpha_{p}^{\max }, \tau \alpha_{d}^{\max }, 1\right\}
\end{aligned}
$$

onde o escalar $\tau \in(0,1)$ é um valor determinado empiricamente, dado por $\tau=0,9995$, ou de acordo com Wright (1995), pode ser calculado da fórmula $1-1 /(9 \sqrt{\mathrm{r}})$, onde r é o número de restrições de desigualdade do problema.

Uma etapa muito importante no algoritmo primal-dual barreira logarítmica é a escolha inicial do parâmetro de barreira. A condição $\nabla_{\mathrm{x}} \mathrm{FL}=0$ sugere que $\mu$ pode ser reduzido com base no gap da complementaridade, como visto em Torres e Quintana (1998).

\subsubsection{ALGORITMO}

i) Dado o problema (3.1), construa a função Lagrangiana (3.10);

ii) Faça $k=0$ e dê uma estimativa inicial para $\mu^{\mathrm{k}}$ e $S^{\mathrm{k}}=\left(\mathrm{x}^{\mathrm{k}}, \mathrm{z}^{\mathrm{k}},\left(\lambda^{\mathrm{E}}\right)^{\mathrm{k}},\left(\lambda^{\mathrm{I}}\right)^{\mathrm{k}}\right)^{\mathrm{T}}$ que satisfaça as condições propostas;

iii) Obtenha o sistema $\mathrm{HS}=-\nabla_{\mathrm{X}} \mathrm{FL}$ e resolva-o;

iv) Calcule os passos primais e duais e atualize $\mathrm{d}^{\mathrm{k}}$ utilizando (3.12);

v) Se a norma do gradiente for menor que uma precisão $\varepsilon$ vá para o passo vi. Caso contrário volte para o passo iii;

vi) Se as condições de KKT são satisfeitas então pare. Caso contrário vá para o passo vii;

vii) Atualize $\mu$ utilizando uma heurística. Faça $k=k+1$ e retorne ao passo iii.

Um ponto inicial estritamente factível não é obrigatório, mas as condições $\mathrm{z}>0$ e $\lambda^{\mathrm{I}}>0$ devem ser satisfeitas em todos os pontos. O processo de otimização termina quando as condições de KKT são satisfeitas. 


\subsubsection{DIFICULDADES COMPUTACIONAIS}

Uma das dificuldades encontradas no método PDBL é a seleção de um ponto inicial factível. Em muitos problemas, isso pode ser trabalhoso. Também, em virtude da estrutura da função barreira, para valores pequenos de $\mu$, o método PDBL pode ter sérios problemas de mal condicionamento e erros de arredondamento, quando o ótimo se aproxima. As escolhas do parâmetro de barreira e do fator de barreira podem comprometer o processo de otimização.

\subsection{MÉTODO DE BARREIRA MODIFICADA}

Polyak, em 1992, desenvolveu uma teoria de métodos da função barreira modificada (FBM). Estes métodos combinam a função Lagrangiana clássica e a função barreira clássica (FBC) buscando explorar as melhores propriedades de cada uma dessas funções. A FBM pode ser considerada como uma função Lagrangiana aumentada interior e é utilizada na resolução de problemas restritos. O método de barreira modificada transforma o problema restrito em um irrestrito equivalente, e resolve uma seqüência de problemas irrestritos até atingir o ótimo. Para um melhor entendimento do método e de suas propriedades descreve-se um método de barreira modificada genérico para o problema (3.1), segundo Nash et al. (1994). A cada iteração principal do método de barreira modificada o problema irrestrito:

\section{Minimizar $\mathrm{M}(\mathrm{x}, \lambda, \mu)$}

$\mathrm{X}$

é resolvido onde $\mathrm{M}(\mathrm{x}, \lambda, \mu)=\mathrm{f}(\mathrm{x})-\mu \sum_{\mathrm{i}=1}^{\mathrm{m}_{\mathrm{c}}} \lambda_{\mathrm{i}} \psi\left(\mu^{-1} \mathrm{c}(\mathrm{x})+1\right)$,

e a solução $x^{k}$ é usada para atualizar $\lambda_{\mathrm{i}}$, via $\lambda_{\mathrm{i}}^{\mathrm{k}}=\lambda_{\mathrm{i}} \psi^{\prime}\left(\mu^{-1} \mathrm{c}_{\mathrm{i}}\left(\mathrm{x}^{\mathrm{k}}\right)+1\right), \mathrm{i}=1, \ldots, \mathrm{m}_{\mathrm{c}}$.

Os parâmetros $\lambda_{\mathrm{i}}, \mathrm{i}=1, \ldots, \mathrm{m}_{\mathrm{c}}$, são estimativas dos multiplicadores de Lagrange na solução $x^{*}$. A função $\psi$ é uma função monotônica, estritamente côncava e de classe $\mathrm{C}_{2}$ definida no intervalo $(0,+\infty)$; uma possível escolha é $\psi()=.\ln ($.$) , uma outra é a função$ inversa $\psi()=.1 /($.$) .$ 
Se, por exemplo, $\psi()=.\ln ($.$) a região factível do problema (3.1) é equivalente ao$ conjunto $\left\{\mathrm{x}: \mu \psi\left(\mu^{-1} \mathrm{c}_{\mathrm{i}}(\mathrm{x})+1\right) \geq 0, \mathrm{i}=1, \ldots, \mathrm{m}_{\mathrm{c}}\right\}$.

Desta forma, a função barreira modificada é a Lagrangiana clássica para o problema (3.1) com as restrições expressas de forma equivalente. O uso do termo de barreira $\psi\left(\mu^{-1} \mathrm{C}_{\mathrm{i}}(\mathrm{x})+1\right)$ corresponde a relaxação das restrições de modo que tenham a forma $\mathrm{c}_{\mathrm{i}}(\mathrm{x}) \geq-\mu$.

Esta relaxação representa uma expansão da região factível. Conseqüentemente a “região factível” implícita para o subproblema de barreira modificada varia com o parâmetro de barreira $\mu$.

Diferente da função barreira logarítmica clássica, a função barreira modificada e suas derivadas existem na solução $\mathrm{x}^{*}$ para qualquer parâmetro de barreira, $\mu$, positivo. Em particular, se $\lambda^{*}$ é o vetor dos multiplicadores de Lagrange correspondente a $x^{*}$, e se $\psi()=.\ln ($.$) , então a função barreira modificada tem as seguintes propriedades para$ qualquer $\mu>0$ :

P1. $\quad M\left(x^{*}, \lambda^{*}, \mu\right)=f\left(x^{*}\right)$

P2. $\quad \nabla_{\mathrm{x}} \mathrm{M}\left(\mathrm{x}^{*}, \lambda^{*}, \mu\right)=\nabla \mathrm{f}\left(\mathrm{x}^{*}\right)-\sum_{\mathrm{i}=1}^{\mathrm{mc}} \lambda^{*} \nabla \mathrm{c}_{\mathrm{i}}\left(\mathrm{x}^{*}\right)=0$

P3. $\quad \nabla_{\mathrm{x}}^{2} \mathrm{M}\left(\mathrm{x}^{*}, \lambda^{*}, \mu\right)=\nabla^{2} \mathrm{f}\left(\mathrm{x}^{*}\right)-\sum_{\mathrm{i}=1}^{\mathrm{mc}} \lambda_{\mathrm{i}}^{*} \nabla^{2} \mathrm{c}_{\mathrm{i}}\left(\mathrm{x}^{*}\right)+\mu \nabla \mathrm{c}\left(\mathrm{x}^{*}\right) \operatorname{diag}\left(\lambda^{*}\right)^{\mathrm{T}} \nabla \mathrm{c}\left(\mathrm{x}^{*}\right)^{\mathrm{T}}$

Quando o problema é de programação convexa, segue de P2 que

P4. $\quad \mathrm{x}^{*}=\arg \min \left\{\mathrm{M}\left(\mathrm{x}, \lambda^{*}, \mu\right)\right\}$, para qualquer $\mu>0$.

Isso significa que se os multiplicadores de Lagrange ótimos são conhecidos, pode-se resolver o problema restrito (3.1) usando um único problema de otimização irrestrito, independente do valor do parâmetro de barreira. Polyak (1992) mostrou que se 
os multiplicadores de Lagrange iniciais são positivos, e os parâmetros de barreira são menores que um valor limite $\bar{\mu}$, o método converge.

Nesse mesmo trabalho, Polyak apresenta três tipos de funções barreira modificadas: uma para a função barreira de Carrol, outra para a função barreira de Frisch e a função barreira Shifted. As funções introduzidas por Frisch, vistas em (3.6), e Carrol, encontradas em (3.5), são as funções barreira mais conhecidas. No entanto, essas funções têm sérias desvantagens porque elas, bem como suas derivadas, não existem em $\mathrm{x}^{*}$ e essas funções vão para infinito quando $\mathrm{x} \rightarrow \mathrm{x}^{*}$. Considerando isto, Polyak definiu as funções barreira de Frisch e Carrol modificadas, estas funções associadas ao problema (3.1) serão mostradas a seguir.

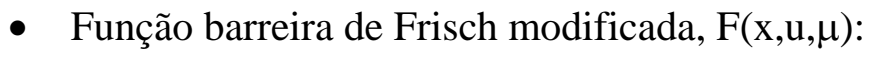

$F(x, \lambda, \mu)= \begin{cases}f(x)-\mu \sum_{i=1}^{m_{c}} \lambda_{i} \ln \left(\mu^{-1} c_{i}(x)+1\right), & \text { se } x \in \operatorname{int} \Omega_{\mu} \\ \infty, & \text { se } x \notin \operatorname{int} \Omega_{\mu},\end{cases}$

- Função barreira de Carrol modificada, $\mathrm{C}(\mathrm{x}, \mathrm{u}, \mu)$ :

$C(x, \lambda, \mu)= \begin{cases}f(x)+\mu \sum_{i=1}^{m_{c}} \lambda_{i}\left[\left(\mu^{-1} c_{i}(x)+1\right)^{-1}-1\right] & \text { se } x \in \operatorname{int} \Omega_{\mu} \\ \infty, & \text { se } x \notin \operatorname{int} \Omega_{\mu},\end{cases}$

onde: int é a parte interior do conjunto e $\Omega_{\mu}$ é o conjunto relaxado dado por: $\Omega_{\mu}=\left\{\mathrm{x}: \mu^{-1} \mathrm{C}_{\mathrm{i}}(\mathrm{x})+1 \geq 0, \mathrm{i}=1, \ldots, \mathrm{m}_{\mathrm{c}}\right\}$. 
Com a adição de um fator de deslocamento (de valor 1) dentro do termo logarítmico das funções barreira modificadas (3.13) e (3.14), a convergência finita nos métodos do tipo barreira foi alcançada, tais funções tornam explícito o uso do multiplicador de Lagrange, $\lambda_{\mathrm{i}}$. O esquema de atualização desses multiplicadores é de complexidade computacional muito baixa. O algoritmo da função barreira modificada possui uma propriedade de convergência finita ao invés de assintótica como no método da função barreira clássica. Isto significa que a solução ótima encontrada no método da FBM pode, de fato, estar na fronteira da região factível, o que não acontece com a FBC, onde a solução somente pode estar próxima à fronteira, mas nunca alcançá-la. Conseqüentemente, as restrições tratadas pela FBM podem ser nulas, diferente da FBC. Outra propriedade do método da FBM é que o parâmetro de barreira, $\mu$, não precisa estar muito próximo de zero para alcançar a solução, desde que, os multiplicadores de Lagrange corretos, $\lambda_{\mathrm{j}}$, sejam obtidos. Assim, o condicionamento da Hessiana é fortemente melhorado.

Neste trabalho utiliza-se a função barreira Frisch modificada (3.13), isto é, a logarítmica. Os passos do método de barreira modificada utilizando essa função aplicada ao problema (3.13), conforme Polyak (1992) são:

Minimiza-se (3.13) com relação a x e satisfaz-se a condição:

$$
\nabla f(x)-\sum_{i=1}^{m_{c}} \frac{\lambda_{i}}{\mu^{-1} c_{i}(x)+1} \nabla c_{i}(x)=0
$$

Aplica-se o método de Newton para solucionar a equação não-linear (3.15). Dessa forma, tem-se a seguinte equação:

$$
\nabla^{2} \mathrm{f}(\mathrm{x})-\sum_{\mathrm{i}=1}^{\mathrm{m}_{\mathrm{c}}} \frac{\lambda_{\mathrm{i}}}{\mu^{-1} \mathrm{c}_{\mathrm{i}}(\mathrm{x})+1} \nabla^{2} \mathrm{c}_{\mathrm{i}}-\sum_{\mathrm{i}=1}^{\mathrm{m}_{\mathrm{c}}} \frac{\lambda_{\mathrm{i}}}{\left(\mu^{-1} \mathrm{c}_{\mathrm{i}}(\mathrm{x})+1\right)^{2}} \nabla \mathrm{c}_{\mathrm{i}}(\mathrm{x}) \nabla \mathrm{c}_{\mathrm{i}}(\mathrm{x})^{\mathrm{T}}=0
$$

Reescrevendo a equação (3.16) de forma simplificada, tem-se o seguinte sistema: 


$$
\nabla_{\mathrm{xx}}^{2} \mathrm{~F}(\mathrm{x}, \lambda, \mu) \mathrm{sx}=-\nabla_{\mathrm{x}} \mathrm{F}(\mathrm{x}, \lambda, \mu)
$$

em que sx é o vetor de correção.

Atualiza-se o vetor $\mathrm{x}$ por:

$$
x^{k+1}=x^{k}+\rho s x
$$

onde $\rho>0$ é o tamanho do passo o qual é encontrado através da regra de GoldsteinArmijo conforme Nocedal e Wright (1999).

A equação (3.15) sugere a seguinte regra para atualização do vetor das estimativas dos multiplicadores de Lagrange:

$$
\lambda_{i}^{\mathrm{k}+1}=\frac{\lambda_{\mathrm{i}}^{\mathrm{k}}}{\mu^{(\mathrm{k}+1)^{-1}} \mathrm{c}_{\mathrm{i}}\left(\mathrm{x}^{(\mathrm{k}+1)}\right)+1}, \mathrm{i}=1, \ldots, \mathrm{m}_{\mathrm{c}} .
$$

\subsubsection{ALGORITMO}

i) Dado o problema (3.1), construa a função barreira modificada (3.13);

ii) Faça $\mathrm{k}=0, \lambda^{0}=(1, . ., 1)$ e dê uma estimativa inicial para $\mathrm{x}^{0}$, e $\mu^{0}>0$;

iii) Construa o sistema (3.17) e resolva-o;

iv) Atualize $x^{k}$ utilizando (3.18) e se $x^{k}$ satisfaz as condições de Goldstein-Armijo, vá para o passo v. Caso contrário, retorne ao passo iii;

v) Se a norma do vetor gradiente for menor que uma precisão $\varepsilon$ vá para o passo vi. Caso contrário volte para o passo iii;

vi) Se $\mathrm{x}^{\mathrm{k}+1}$ satisfaz KKT, pare. Caso contrário, vá para o passo vii;

vii) Atualize o vetor das estimativas dos multiplicadores de Lagrange, u, usando (3.19) e $\mu$ utilizando uma heurística. Faça k=k+1 e retorne ao passo iii.

Observa-se que um ponto inicial factível não é obrigatório, mas a condição inicial $c_{i}\left(x^{0}\right)>-\mu$ deve ser satisfeita. 


\subsubsection{DIFICULDADES COMPUTACIONAIS}

Uma das dificuldades encontradas no método de barreira modificada é o cálculo do tamanho do passo para atualização das variáveis, pois caso isso seja feito sem um critério de parada bem fundamentado o processo computacional pode consumir tempo e pode complicar-se. A escolha do parâmetro de barreira inicial e a sua forma de atualização podem interferir no processo de otimização.

No próximo capítulo apresenta-se o método da função Lagrangiana barreira modificada/penalidade. 


\section{CAPÍTULO 4}

\section{O MÉTODO DA FUNÇÃO LAGRANGIANA BARREIRA MODIFICADA/PENALIDADE}

Neste capítulo apresentamos uma nova abordagem do método da função barreira modificada. Nesta abordagem, as restrições canalizadas são tratadas pela função barreira modificada apresentada por Polyak (1992) ou por uma extrapolação quadrática proposta por Breitfeld e Shanno (1994b), isto é, uma função penalidade quadrática. As restrições de igualdade são tratadas pela função Lagrangiana. O método de Newton é usado para resolver um sistema não-linear, proveniente da aplicação das condições de otimalidade de $1^{\mathrm{a}}$ ordem. É apresentado, também, o algoritmo do método.

\subsection{APRESENTAÇÃO DO PROBLEMA}

Considere o seguinte problema de programação não-linear (PPNL):

$$
\begin{array}{ll}
\text { Minimizar } & f(x) \\
\text { sujeito a: } & \left\{\begin{array}{l}
\underline{c_{i}} \leq c_{i}(x) \leq \overline{c_{i}}, i=1, \ldots, m_{c} \\
h_{j}(x)=0, \\
\underline{x_{k}} \leq x_{k} \leq \overline{x_{k}}, k=1, \ldots, m_{h}
\end{array}\right.
\end{array}
$$


em que $c_{i}(x)$ representa as restrições de desigualdade canalizadas; $h_{j}(x)$, as restrições de igualdade; $\underline{x_{k}}$ e $\overline{x_{k}}$ são os limites inferior e superior impostos à variável $x_{k}$ (canalizações); e $\underline{c_{i}}$ e $\overline{c_{i}}$ são os respectivos limites inferior e superior impostos às restrições $\mathrm{C}_{\mathrm{i}}(\mathrm{x})$.

\subsection{A FUNÇÃO LAGRANGIANA BARREIRA MODIFICADA/ PENALIDADE}

Para construir a função Lagrangiana barreira modificada/penalidade (FLBMP) nós transformarmos o PPNL (4.1) num problema equivalente, somente com restrições de igualdade, seguindo 2 passos:

Passo 1: Introduzimos a variável de folga $\mathrm{z}_{\mathrm{i}}, \mathrm{i}=1, \ldots, \mathrm{m}_{\mathrm{c}}$, nas restrições de desigualdade canalizadas do problema (4.1) de tal forma que $\mathrm{c}_{\mathrm{i}}(\mathrm{x})-\mathrm{z}_{\mathrm{i}}=0$ e separamos cada uma das restrições de desigualdade canalizadas, em duas restrições de desigualdade, como segue:

$$
\begin{gathered}
\underline{\mathrm{c}_{\mathrm{i}}} \leq \mathrm{c}_{\mathrm{i}}(\mathrm{x}) \leq \overline{\mathrm{c}_{\mathrm{i}}} \\
\underline{\mathrm{c}_{\mathrm{i}}}-\mathrm{z}_{\mathrm{i}} \leq \mathrm{c}_{\mathrm{i}}(\mathrm{x})-\mathrm{z}_{\mathrm{i}} \leq \overline{\mathrm{c}_{\mathrm{i}}}-\mathrm{z}_{\mathrm{i}} \\
\text { como } \mathrm{c}_{\mathrm{i}}(\mathrm{x})-\mathrm{z}_{\mathrm{i}}=0, \text { temos: } \\
\underline{\mathrm{c}_{\mathrm{i}}}-\mathrm{z}_{\mathrm{i}} \leq 0 \leq \overline{\mathrm{c}_{\mathrm{i}}}-\mathrm{z}_{\mathrm{i}}
\end{gathered}
$$

o que equivale a:

$$
\left\{\begin{array}{l}
\frac{\mathrm{c}_{\mathrm{i}}}{\underline{\bar{c}}}-\mathrm{z}_{\mathrm{i}} \leq 0 \\
\overline{\mathrm{c}_{\mathrm{i}}}-\mathrm{z}_{\mathrm{i}} \geq 0
\end{array}\right.
$$

ou:

$$
\left\{\begin{array}{l}
\mathrm{z}_{\mathrm{i}}-\underline{\mathrm{c}_{\mathrm{i}}} \geq 0 \\
\overline{\mathrm{c}_{\mathrm{i}}}-\mathrm{z}_{\mathrm{i}} \geq 0
\end{array}\right.
$$

Em seguida, separamos as canalizações das variáveis $x_{k}$ em duas restrições de desigualdade e com isso, o nosso PPNL (4.1) toma a seguinte forma: 
Minimizar $\mathrm{f}(\mathrm{x})$

sujeito a: $\quad h_{j}(x)=0, j=1, \ldots, m_{h}$

$$
\begin{aligned}
& c_{i}(x)-z_{i}=0 \\
& z_{i}-\underline{c_{i}} \geq 0 \\
& \overline{c_{i}}-z_{i} \geq 0, i=1, \ldots, m_{c} \\
& x_{k}-\underline{x_{k}} \geq 0 \\
& \overline{x_{k}}-x_{k} \geq 0, k=1, \ldots, m_{x}
\end{aligned}
$$

Esta formulação faz com que o PPNL que tinha $\mathrm{m}_{\mathrm{c}}$ restrições de desigualdade canalizadas, $m_{h}$ restrições de igualdade e $m_{x}$ canalizações das variáveis $x_{k}$, passe a ter $2 m_{c}+2 m_{x}$ restrições de desigualdade e $m_{h}+m_{c}$ restrições de igualdade.

Passo 2: Construímos a função barreira modificada/penalidade, FBMP, associada à (4.2) como segue:

$$
\text { FBMP }=\mathrm{f}(\mathrm{x})-\mu \sum_{\mathrm{k}=1}^{\mathrm{m}_{\mathrm{x}}}\left[\mathrm{v}_{\mathrm{k}}^{\mathrm{I}} \phi\left(\mathrm{x}_{\mathrm{k}}-\underline{\mathrm{x}_{\mathrm{k}}}\right)+\mathrm{v}_{\mathrm{k}}^{\mathrm{s}} \phi\left(\overline{\mathrm{x}_{\mathrm{k}}}-\mathrm{x}_{\mathrm{k}}\right)\right]-\mu \sum_{\mathrm{i}=1}^{\mathrm{m}_{\mathrm{C}}}\left[\xi_{\mathrm{i}}^{\mathrm{I}} \phi\left(\mathrm{z}_{\mathrm{i}}-\underline{\mathrm{c}_{\mathrm{i}}}\right)+\xi_{\mathrm{i}}^{\mathrm{s}} \phi\left(\overline{\mathrm{c}_{\mathrm{i}}}-\mathrm{z}_{\mathrm{i}}\right)\right]
$$

em que $\mathrm{v}_{\mathrm{k}}^{\mathrm{I}}, \mathrm{v}_{\mathrm{k}}^{\mathrm{S}}, \xi_{\mathrm{i}}^{\mathrm{I}}, \xi_{\mathrm{i}}^{\mathrm{S}}$ são os multiplicadores de Lagrange das restrições de desigualdade; $\mathrm{z}_{\mathrm{i}}$ é a variável de folga adicionada às restrições de desigualdade canalizadas; $\mu$ é o parâmetro de barreira e $\phi(x)$ é um termo de barreira modificada/penalidade. Para simplificar a notação, as restrições de desigualdade $\left(x_{k}-\underline{x_{k}}, \overline{x_{k}}-x_{k}, z_{i}-\underline{c_{i}}\right.$ e $\left.\overline{c_{i}}-z_{i}\right)$ serão denotadas, a partir de agora, como $g_{i}(x, z)$, $\mathrm{i}=1, \ldots, \mathrm{m}, \mathrm{com} \mathrm{m}=2 \mathrm{~m}_{\mathrm{c}}+2 \mathrm{~m}_{\mathrm{x}}$. A função $\phi$ é definida por:

$$
\phi\left(g_{i}(x, z)\right)=\ln \left(s_{i}+\frac{g_{i}(x, z)}{\mu}\right), i=1,2, \ldots, m, \text { se } g_{i}(x, z) \geq-\beta s_{i} \mu
$$

$\mathrm{ou}$

$$
\phi\left(g_{i}(x, z)\right)=Q\left(g_{i}(x, z)\right), i=1,2, \ldots, m, \text { se } g_{i}(x, z)<-\beta s_{i} \mu
$$




$$
Q\left(g_{i}(x, z)\right)=\frac{1}{2} q_{a, i}\left(g_{i}(x, z)\right)^{2}+q_{b, i} g_{i}(x, z)+q_{c, i}, i=1,2, \ldots, m,
$$

é uma função penalidade (ou extrapolação quadrática), $\beta$ é um parâmetro que representa a tolerância da aproximação da região factível e s é um parâmetro shift.

Como o parâmetro $\beta \rightarrow 1$, as funções $\phi\left(\mathrm{g}_{\mathrm{i}}(\mathrm{x}, \mathrm{z})\right)$ aproximam-se das singularidades dos termos logaritmos em (4.4). Os coeficientes da função penalidade (ou extrapolação quadrática), $\mathrm{q}_{\mathrm{a}, \mathrm{i}}, \mathrm{q}_{\mathrm{b}, \mathrm{i}}$ e $\mathrm{q}_{\mathrm{c}, \mathrm{i}}$, apresentados em (4.6), são calculados de forma que esta função tenha o seu valor, bem como os das suas derivadas primeira e segunda ordem coincidentes com os da função logarítmica (4.4), no ponto $g(x, z)=-\beta s \mu$, como foi proposto pelos pesquisadores Bental et al. (1992), e Breitfeld e Shanno (1993). Logo, sendo $B_{m}(g(x, z))=\ln \left(s+\frac{g(x, z)}{\mu}\right)$, temos que satisfazer as seguintes igualdades:

$$
\begin{aligned}
& \mathrm{Q}(\mathrm{g}(\mathrm{x}, \mathrm{z}))=\mathrm{B}_{\mathrm{m}}(\mathrm{g}(\mathrm{x}, \mathrm{z})) \text {, isto é, } \frac{1}{2} \mathrm{q}_{\mathrm{a}}(\mathrm{g}(\mathrm{x}, \mathrm{z}))^{2}+\mathrm{q}_{\mathrm{b}} \mathrm{g}(\mathrm{x}, \mathrm{z})+\mathrm{q}_{\mathrm{c}}=\ln \left(\mathrm{s}+\frac{\mathrm{g}(\mathrm{x}, \mathrm{z})}{\mu}\right) \\
& \mathrm{Q}^{\prime}(\mathrm{g}(\mathrm{x}, \mathrm{z}))=\mathrm{B}_{\mathrm{m}}{ }^{\prime}(\mathrm{g}(\mathrm{x}, \mathrm{z})) \text {, isto é, } \mathrm{q}_{\mathrm{a}} \mathrm{g}(\mathrm{x}, \mathrm{z})+\mathrm{q}_{\mathrm{b}}=\frac{1}{\mu \mathrm{s}+\mathrm{g}(\mathrm{x}, \mathrm{z})} \\
& \mathrm{Q}^{\prime \prime}(\mathrm{g}(\mathrm{x}, \mathrm{z}))=\mathrm{B}_{\mathrm{m}}{ }^{\prime \prime}(\mathrm{g}(\mathrm{x}, \mathrm{z})) \text {, isto é, } \mathrm{q}_{\mathrm{a}}=-\frac{1}{(\mu \mathrm{s}+\mathrm{g}(\mathrm{x}, \mathrm{z}))^{2}}
\end{aligned}
$$

Substituindo $\mathrm{g}(\mathrm{x})=-\beta \mathrm{s} \mu$ em (4.9), temos:

$$
\mathrm{q}_{\mathrm{a}}=-\frac{1}{(\mu \mathrm{s}(1-\beta))^{2}}
$$

Substituindo $g(x)=-\beta s \mu$ e (4.10) em (4.8), temos:

$$
-\frac{1}{(\mu \mathrm{s}(1-\beta))^{2}}(-\beta s \mu)+\mathrm{q}_{\mathrm{b}}=\frac{1}{\mu \mathrm{s}-\beta \mathrm{s} \mu}
$$




$$
\mathrm{q}_{\mathrm{b}}=\frac{1}{(\mu \mathrm{s}(1-\beta))}-\frac{\beta \mathrm{s} \mu}{(\mu \mathrm{s}(1-\beta))^{2}}=\frac{\mu \mathrm{s}-\mu \mathrm{s} \beta-\beta \mathrm{s} \mu}{(\mu \mathrm{s}(1-\beta))^{2}}=\frac{1-2 \beta}{\mu \mathrm{s}(1-\beta)^{2}}
$$

Substituindo $\mathrm{g}(\mathrm{x})=-\beta \mathrm{s} \mu$, (4.10) e (4.11) em (4.7), temos:

$$
\frac{1}{2}\left(-\frac{1}{(\mu \mathrm{s}(1-\beta))^{2}}\right)(-\beta s \mu)^{2}+\left(\frac{1-2 \beta}{\mu \mathrm{s}(1-\beta)^{2}}\right)(-\beta s \mu)+\mathrm{q}_{\mathrm{c}}=\ln \left(\mathrm{s}-\frac{\beta \mathrm{s} \mu}{\mu}\right),
$$

ou

$$
\frac{-\beta^{2}}{2(1-\beta)^{2}}+\frac{2 \beta^{2}-\beta}{(1-\beta)^{2}}+\mathrm{q}_{\mathrm{c}}=\ln (\mathrm{s}[1-\beta])
$$

isolando $\mathrm{q}_{\mathrm{c}}$, temos,

$$
\mathrm{q}_{\mathrm{c}}=\frac{\beta^{2}-4 \beta^{2}+2 \beta}{2(1-\beta)^{2}}+\ln (\mathrm{s}[1-\beta])
$$

ou

$$
\mathrm{q}_{\mathrm{c}}=\frac{\beta(2-3 \beta)}{2(1-\beta)^{2}}+\ln (\mathrm{s}[1-\beta])
$$

Portanto, as equações (4.10), (4.11) e (4.12) nos fornecem os coeficientes da função extrapolação quadrática Q(x), vista em (4.6), para a FLBMP, ou seja:

$$
\begin{aligned}
& \mathrm{q}_{\mathrm{a}, \mathrm{i}}=\frac{-1}{\left(\mathrm{~s}_{\mathrm{i}} \mu(1-\beta)\right)^{2}} \\
& \mathrm{q}_{\mathrm{b}, \mathrm{i}}=\frac{1-2 \beta}{\mathrm{s}_{\mathrm{i}} \mu(1-\beta)^{2}} \\
& \mathrm{q}_{\mathrm{c}, \mathrm{i}}=\frac{\beta(2-3 \beta)}{2(1-\beta)^{2}}+\ln \left(\mathrm{s}_{\mathrm{i}}(1-\beta)\right)
\end{aligned}
$$


Com isso, o PPNL (4.2) torna-se um problema de minimização somente com restrições de igualdade, com a seguinte forma:

$\min _{x, z}$ FBMP

sujeito a: $\quad h_{j}(x)=0, j=1, \ldots, m_{h}$

$$
c_{i}(x)-z_{i}=0, i=1, \ldots, m_{c} \text {. }
$$

Para transformarmos (4.16) em um problema irrestrito equivalente, construímos a função Lagrangiana barreira modificada/penalidade associada a ele da seguinte forma:

$$
\begin{aligned}
\text { FLBMP }= & f(x)-\mu \sum_{k=1}^{m_{x}}\left[v_{k}^{I} \phi\left(x_{k}-\underline{x_{k}}\right)+v_{k}^{\mathrm{s}} \phi\left(\overline{x_{k}}-x_{k}\right)\right]-\mu \sum_{i=1}^{m_{C}}\left[\xi_{i}^{I} \phi\left(z_{i}-\underline{c_{i}}\right)+\xi_{i}^{\mathrm{S}} \phi\left(\overline{c_{i}}-z_{i}\right)\right]+ \\
& +\sum_{j=1}^{m_{h}} \lambda_{j}^{E} h_{j}(x)+\sum_{i=1}^{m_{c}} \lambda \lambda_{i}^{I}\left(c_{i}(x)-z_{i}\right)
\end{aligned}
$$

em que $\lambda_{\mathrm{j}}^{\mathrm{E}}$ e $\lambda_{\mathrm{i}}^{\mathrm{I}}$ são os multiplicadores de Lagrange para as restrições de igualdade do problema (4.2).

Com isso, transformamos o PPNL (4.16) no seguinte PPNL irrestrito:

$\min _{\mathrm{x}, \mathrm{z}}$ FLBMP

\subsection{O MÉTODO DA FUNÇÃO LAGRANGIANA BARREIRA MODIFICADA /PENALIDADE}

O método da FLBMP é composto por 2 ciclos: um externo e um interno. No ciclo externo o PPNL é convertido em um problema irrestrito, como visto na seção 4.2. Os multiplicadores de Lagrange associados com as restrições de desigualdade $\left(\mathrm{v}_{\mathrm{k}}{ }^{\mathrm{I}}, \mathrm{v}_{\mathrm{k}} \mathrm{S}\right.$, $\left.\xi_{\mathrm{i}}^{\mathrm{I}}, \xi_{\mathrm{i}}^{\mathrm{S}}\right)$ e o parâmetro de barreira são determinados. No ciclo interno resolve-se o sistema não-linear obtido pela aplicação das condições de otimalidade de $1^{\mathrm{a}}$ ordem à função (4.17). O método de Newton é usado na atualização das variáveis primais, x e z, 
e as duais $\left(\lambda_{j}^{E}\right.$ e $\left.\lambda_{i}^{I}\right)$, cujo passo é determinado por um procedimento de busca linear. Logo que a convergência satisfatória é atingida no ciclo interno, o novo conjunto de variáveis é usado para controlar a convergência do ciclo externo. Se a convergência externa não for alcançada, os multiplicadores associados às restrições de desigualdade $\left(\mathrm{v}_{\mathrm{k}}{ }^{\mathrm{I}}, \mathrm{v}_{\mathrm{k}}{ }^{\mathrm{S}}, \xi_{\mathrm{i}}{ }^{\mathrm{I}}, \xi_{\mathrm{i}}{ }^{\mathrm{S}}\right)$ são atualizados e uma nova atualização é iniciada no ciclo interno.

A seguir, descrevemos detalhadamente cada uma das etapas realizadas nos 2 ciclos.

\subsubsection{O CICLO INTERNO DO MÉTODO DA FLBMP (ITERAÇÃO DE NEWTON)}

Nesta subseção, nós apresentamos o ciclo interno do método da FLBMP juntamente com o procedimento de busca linear utilizado. Uma iteração deste ciclo envolve a avaliação de uma Hessiana exata e de um gradiente. A factibilidade é verificada para as restrições de desigualdade. Se alguma delas não satisfizer a factibilidade em algum passo da busca, significa que o termo logarítmico de barreira modificada associado a esta restrição ultrapassou, ou está muito próximo da sua assíntota, dificultando, ou impossibilitando o cálculo do logaritmo. A estratégia usada para evitar esse fato é o uso da extrapolação quadrática (4.5), mudando o termo logarítmico para um termo quadrático.

Aplicando as condições de otimalidade de $1^{\mathrm{a}}$ ordem na FLBMP (4.17), obtemos um sistema não-linear, como segue:

$$
\left\{\begin{array}{l}
\frac{\partial \mathrm{FLBMP}}{\partial \mathrm{x}}=0 \\
\frac{\partial \mathrm{FLBMP}}{\partial \mathrm{z}}=0 \\
\frac{\partial \mathrm{FLBMP}}{\partial \lambda^{\mathrm{E}}}=0 \\
\frac{\partial \mathrm{FLBMP}}{\partial \lambda^{\mathrm{I}}}=0
\end{array}\right.
$$


Determinamos a solução do sistema não-linear (4.19) utilizando o método de Newton. A aplicação do método de Newton gera as direções de busca, sx, sz, $s \lambda^{\mathrm{E}}$ e $s \lambda^{\mathrm{I}}$, as quais são utilizadas para a atualização das variáveis do sistema e resulta num sistema matricial, que, em sua forma simplificada, é representado por:

$$
\mathrm{H}_{\text {FLBMP }} \mathrm{S}=-\mathrm{G}_{\text {FLBMP }}
$$

em que $G_{\text {FLBMP }}$ é o gradiente da FLBMP (4.17), H HLBMP é a Hessiana da FLBMP (4.17) e $S=\left(s x, s z, s \lambda^{\mathrm{E}}, \mathrm{s} \lambda^{\mathrm{I}}\right)$ é o vetor direção de busca.

O G GLBMP é definido como segue:

$\mathrm{G}_{\mathrm{FLBMP}}=\nabla \mathrm{FLBMP}\left(\mathrm{x}, \mathrm{z}, \lambda^{\mathrm{E}}, \lambda^{\mathrm{I}}\right)=\left[\left(\frac{\partial \mathrm{FLBMP}}{\partial \mathrm{x}}\right)^{\mathrm{T}}\left(\frac{\partial \mathrm{FLBMP}}{\partial \mathrm{z}}\right)^{\mathrm{T}}\left(\frac{\partial \mathrm{FLBMP}}{\partial \lambda^{\mathrm{E}}}\right)^{\mathrm{T}}\left(\frac{\partial \mathrm{FLBMP}}{\partial \lambda^{\mathrm{I}}}\right)^{\mathrm{T}}\right]^{\mathrm{T}}$

com:

$\frac{\partial \text { FLBMP }}{\partial \mathrm{x}}=\frac{\partial \mathrm{f}(\mathrm{x})}{\partial \mathrm{x}}-\mu\left(\underset{\mathrm{k}=1,2, \ldots, \mathrm{mx}}{\operatorname{vetor}}\left[\mathrm{v}_{\mathrm{k}}^{\mathrm{I}} \phi^{\prime}\left(\mathrm{x}_{\mathrm{k}}-\underline{\mathrm{x}_{\mathrm{k}}}\right)-\mathrm{v}_{\mathrm{k}}^{\mathrm{S}} \phi^{\prime}\left(\overline{\mathrm{x}_{\mathrm{k}}}-\mathrm{x}_{\mathrm{k}}\right)\right]\right)+\left[\lambda^{\mathrm{E}}\right]^{\mathrm{T}}\left[\frac{\partial \mathrm{h}(\mathrm{x})}{\partial \mathrm{x}}\right]+\left[\lambda^{\mathrm{I}}\right]^{\mathrm{T}}\left[\frac{\partial \mathrm{c}(\mathrm{x})}{\partial \mathrm{x}}\right]$

$\frac{\partial \text { FLBMP }}{\partial z}=-\mu\left(\underset{i=1,2, \ldots, m c}{\operatorname{vetor}}\left[\xi_{i}^{I} \phi^{\prime}\left(z_{i}-\underline{c_{i}}\right)-\xi_{i}^{\mathrm{s}} \phi^{\prime}\left(\overline{c_{i}}-z_{i}\right)\right]\right)-\lambda^{I}$

$\frac{\partial \text { FLBMP }}{\partial \lambda^{\mathrm{E}}}=\mathrm{h}(\mathrm{x})$

$\frac{\partial \text { FLBMP }}{\partial \lambda^{\mathrm{I}}}=\mathrm{c}(\mathrm{x})-\mathrm{z}$

em que: 


$$
\left\{\begin{array}{l}
\phi^{\prime}\left(g_{i}(x, z)\right)=\frac{1}{\mu s_{i}+g_{i}(x, z)}, i=1,2, \ldots, m, \text { se } g_{i}(x, z) \geq-\beta s_{i} \mu \\
\phi^{\prime}\left(g_{i}(x, z)\right)=q_{a, i} g_{i}(x, z)+q_{b, i}, i=1,2, \ldots, m, \text { se } g_{i}(x, z)<-\beta s_{i} \mu
\end{array}\right.
$$

A Hessiana da FLBMP (4.17) é definida por:

$$
\mathrm{H}_{\mathrm{FLBMP}}=\left(\begin{array}{cccc}
\frac{\partial^{2} \mathrm{FLBMP}}{\partial \mathrm{x}^{2}} & 0 & \left(\frac{\partial \mathrm{h}(\mathrm{x})}{\partial \mathrm{x}}\right)^{\mathrm{T}} & \left(\frac{\partial \mathrm{c}(\mathrm{x})}{\partial \mathrm{x}}\right)^{\mathrm{T}} \\
0 & \frac{\partial^{2} \mathrm{FLBMP}}{\partial \mathrm{z}^{2}} & 0 & -\mathrm{I}_{\mathrm{m}_{\mathrm{c}}} \\
\frac{\partial \mathrm{h}(\mathrm{x})}{\partial \mathrm{x}} & 0 & 0 & 0 \\
\frac{\partial \mathrm{c}(\mathrm{x})}{\partial \mathrm{z}} & -\mathrm{I}_{\mathrm{m}_{\mathrm{c}}} & 0 & 0
\end{array}\right)
$$

com:

$\frac{\partial^{2} \text { FLBMP }}{\partial x^{2}}=\frac{\partial^{2} f(x)}{\partial x^{2}}-\mu \operatorname{diag}_{k=1,2, \ldots, m_{x}}\left[v_{k}^{I} \phi^{\prime \prime}\left(x_{k}-\underline{x_{k}}\right)+v_{i}^{S} \phi^{\prime \prime}\left(\overline{x_{k}}-x_{k}\right)\right]+\left[\lambda^{E}\right]^{T}\left[\frac{\partial^{2} h(x)}{\partial x^{2}}\right]+\left[\lambda^{I}\right]^{T}\left[\frac{\partial^{2} c(x)}{\partial x^{2}}\right]$

$\frac{\partial^{2} \text { FLBMP }}{\partial z^{2}}=-\mu \operatorname{diag}\left[\xi_{i=1,2, \ldots, m_{c}}^{L} \phi^{\prime \prime}\left(z_{i}-\underline{c_{i}}\right)+\xi_{j}^{s} \phi^{\prime \prime}\left(\overline{c_{i}}-z_{i}\right)\right]$

em que

$$
\left\{\begin{array}{l}
\phi^{\prime \prime}\left(g_{i}(x, z)\right)=-\frac{1}{\left(\mu s_{i}+g_{i}(x, z)\right)^{2}}, i=1,2, \ldots, \text { m, se } g_{i}(x, z) \geq-\beta s_{i} \mu \\
\phi^{\prime \prime}\left(g_{i}(x, z)\right)=q_{a, i}, i=1,2, \ldots, m, \text { se } g_{i}(x, z)<-\beta s_{i} \mu
\end{array}\right.
$$

Consequentemente, os novos valores atualizados das variáveis dentro do ciclo interno são dados por: 


$$
\mathrm{X}^{\mathrm{p}+1}=\mathrm{X}^{\mathrm{p}}+\alpha \mathrm{S}
$$

em que:

$\mathrm{X}^{\mathrm{p}}=\left[\mathrm{x}_{1}{ }^{\mathrm{p}} \ldots \mathrm{x}_{\mathrm{mx}}^{\mathrm{p}} \quad \mathrm{z}_{1}^{\mathrm{p}} \ldots \mathrm{z}_{\mathrm{mc}}^{\mathrm{p}} \quad\left(\lambda_{1}^{\mathrm{E}}\right)^{\mathrm{p}} \ldots\left(\lambda_{\mathrm{mx}}{ }^{\mathrm{E}}\right)^{\mathrm{p}} \quad\left(\lambda_{1}^{\mathrm{I}}\right)^{\mathrm{p}} \ldots\left(\lambda_{\mathrm{mc}}{ }^{\mathrm{I}}\right)^{\mathrm{p}}\right]^{\mathrm{T}}$

onde p indica o indexador das iterações internas para a solução do problema de minimização interno e $\alpha$ é o tamanho do passo, o qual é determinado através de uma busca linear, como apresentada na próxima subseção.

\subsubsection{BUSCA LINEAR}

Utilizamos para a busca linear o método de Armijo, que é um método de busca unidimensional finito o qual procura a partir de um ponto $\mathrm{x}^{\mathrm{BL}}$, um ponto $\mathrm{x}^{\mathrm{BL}+1}$, na direção S, tal que a função “decresça”, sem se preocupar em minimizá-la, em que BL indica o indexador de iterações da busca linear. Neste método não se faz necessário a obtenção de um mínimo local para alguma função dependente de $\alpha$ (tamanho do passo), mas apenas uma aproximação mais ou menos precisa. Não se exige convexidade, nem unimodalidade. Para tal, utiliza derivadas no ponto de partida $\mathrm{x}^{\mathrm{BL}}$, devendo garantir neste ponto, ou em cada iteração, que a função decresça, ou seja, nós queremos que:

$$
f\left(x^{p+1}\right) \leq f\left(x^{p}\right)+w \alpha\left(S^{p}\right)^{T} \nabla f\left(x^{p}\right)
$$

em que w é uma constante suave, satisfazendo $0<\mathrm{w}<1$.

O algoritmo de Armijo procura localizar $\alpha$ de forma que a redução da função seja grande. A escolha inicial de $\alpha$ não deve ser muito grande, evitando um comportamento oscilatório do método e nem muito pequeno evitando uma parada prematura do algoritmo. Com isso, ajustaremos o nosso $\alpha_{0}$ (alfa inicial) com o valor 1 . Caso a condição em (4.33) não seja satisfeita, $\alpha$ é atualizado com uma simples redução de acordo com a seqüência: 


$$
\alpha^{\mathrm{BL}}=\frac{\alpha^{\mathrm{BL}-1}}{\delta}
$$

em que $\delta>1$ e é usualmente ajustado para $\delta=2$. Os valores das variáveis primais são atualizados da seguinte forma:

$$
\left(x^{p}\right)^{B L+1}=\left(x^{p}\right)^{B L}+\alpha s^{p}
$$

em que $\mathrm{s}^{\mathrm{p}}=\left(\mathrm{sx}^{\mathrm{p}}, \mathrm{sz}^{\mathrm{p}}\right)$ é a direção de busca somente das variáveis primais, encontrada na resolução do sistema matricial (4.20).

Para a atualização dos multiplicadores de Lagrange associados com as restrições de igualdade, $\lambda_{j}{ }^{\mathrm{E}}$ e $\lambda_{i}{ }^{\mathrm{I}}$ (variáveis duais), encontrados no vetor $\mathrm{X}^{\mathrm{p}}$ em (4.32), usaremos o esquema proposto por CHEN \& VASSILIADIS (2003), o qual é baseado em uma combinação convexa entre os multiplicadores de Lagrange $\left(\left(\lambda_{j}^{\mathrm{E}}\right)^{\mathrm{p}}\right.$ e $\left.\left(\lambda_{i}^{\mathrm{I}}\right)^{\mathrm{p}}\right)$ obtidos no ciclo interno, usando o passo de Newton puro $(\alpha=1)$ e os multiplicadores de Lagrange obtidos dentro da busca linear $\left(\left(\lambda_{j}^{\mathrm{E}}\right)^{\mathrm{BL}}\right.$ e $\left.\left(\lambda_{\mathrm{i}}^{\mathrm{I}}\right)^{\mathrm{BL}}\right)$ :

$$
\begin{aligned}
& \left(\lambda_{j}^{\mathrm{E}}\right)^{\mathrm{BL}+1}=\left(\lambda_{j}^{\mathrm{E}}\right)^{\mathrm{BL}}+\alpha^{\mathrm{BL}}\left(\left(\lambda_{\mathrm{j}}^{\mathrm{E}}\right)^{\mathrm{p}}-\left(\lambda_{j}^{\mathrm{E}}\right)^{\mathrm{BL}}\right) \\
& \left(\lambda_{\mathrm{i}}^{\mathrm{I}}\right)^{\mathrm{BL}+1}=\left(\lambda_{\mathrm{i}}^{\mathrm{I}}\right)^{\mathrm{BL}}+\alpha^{\mathrm{BL}}\left(\left(\lambda_{\mathrm{i}}^{\mathrm{I}}\right)^{\mathrm{p}}-\left(\lambda_{\mathrm{i}}^{\mathrm{I}}\right)^{\mathrm{BL}}\right)
\end{aligned}
$$

Durante a busca linear, essas novas atualizações, $\left(x^{\mathrm{P}}\right)^{\mathrm{BL}+1},\left(\lambda_{j}^{\mathrm{E}}\right)^{\mathrm{BL}+1}$ e $\left(\lambda_{i}^{\mathrm{I}}\right)^{\mathrm{BL}+1}$, serão utilizadas para estimar uma função penalidade mérito para o problema de otimização em lugar da função objetivo $f(x)$ em (4.33) . A função penalidade mérito, como proposta por Chen e Vassiliadis (2003), toma a seguinte forma:

$$
\begin{aligned}
\mathrm{FPM}_{\mathrm{a}}= & \mathrm{f}(\mathrm{x})-\mu \sum_{\mathrm{k}=1}^{\mathrm{m}_{\mathrm{x}}}\left[\mathrm{v}_{\mathrm{k}}^{\mathrm{I}} \phi\left(\mathrm{x}_{\mathrm{k}}-\underline{\mathrm{x}_{\mathrm{k}}}\right)+\mathrm{v}_{\mathrm{k}}^{\mathrm{S}} \phi\left(\overline{\mathrm{x}_{\mathrm{k}}}-\mathrm{x}_{\mathrm{k}}\right)\right]-\mu \sum_{\mathrm{i}=1}^{\mathrm{m}_{\mathrm{C}}}\left[\xi_{\mathrm{i}}^{\mathrm{I}} \phi\left(\mathrm{z}_{\mathrm{i}}-\underline{\mathrm{c}_{\mathrm{i}}}\right)+\xi_{\mathrm{i}}^{\mathrm{S}} \phi\left(\overline{\mathrm{c}_{\mathrm{i}}}-\mathrm{z}_{\mathrm{i}}\right)\right]+ \\
& +\sum_{\mathrm{j}=1}^{\mathrm{m}_{\mathrm{h}}} \max \left[\mathrm{M}_{\mathrm{j}},\left|\lambda_{\mathrm{j}}^{\mathrm{E}}\right|\right]\left|\mathrm{h}_{\mathrm{j}}(\mathrm{x})\right|+\sum_{\mathrm{i}=1}^{\mathrm{m}_{\mathrm{c}}} \max \left[\mathrm{M}_{\mathrm{i}},\left|\lambda_{\mathrm{i}}^{\mathrm{I}}\right|\right]|| \mathrm{c}_{\mathrm{i}}(\mathrm{x})-\mathrm{z}_{\mathrm{i}} \mid
\end{aligned}
$$


em que um termo de penalidade, $\mathrm{M}$, é introduzido para medir a violação das restrições de igualdade. Atualizaremos $\mathrm{M}$ da seguinte forma:

$$
\mathrm{M}^{\mathrm{novo}}=\max \left[\mathrm{M}^{\text {atual }},|\lambda|\right]
$$

em que $\lambda$ representa $\lambda_{j}^{E}$ e $\lambda_{i}^{I}$. O valor usual para inicialização de M é 10 , segundo Chen e Vassiliadis (2003).

Quando a condição (4.33) for satisfeita, o ciclo da busca linear é interrompido, e o vetor gradiente (4.21) e a matriz Hessiana (4.27) serão avaliados para os novos valores encontrados a partir de (4.35), (4.36) e (4.37). Uma nova direção de busca, resolvendo o sistema matricial (4.20) é encontrada, assim como os novos valores das variáveis utilizando (4.31). Esses procedimentos continuam ciclicamente até que um critério de convergência seja atingido. O critério adotado por nós, segundo Chen e Vassiliadis (2003), é dado por:

$$
\left\|\mathrm{G}\left(\mathrm{x}, \mathrm{z}, \lambda^{\mathrm{E}}, \lambda^{\mathrm{I}}\right)\right\| \leq \varepsilon
$$

em que $\mathrm{G}$ é o gradiente da função Lagrangiana barreira modificada/penalidade ou da função Lagrangiana barreira clássica/penalidade (que será vista na seção 4.3.2.3.2), dependendo do método utilizado e $\varepsilon$ é uma precisão.

Quando (4.40) for satisfeito, o ciclo interno é interrompido e inicia-se o ciclo externo. No ciclo externo, o parâmetro de barreira $\mu$ e as estimativas dos multiplicadores de Lagrange $\left(\mathrm{v}_{\mathrm{k}}{ }^{\mathrm{I}}, \mathrm{v}_{\mathrm{k}}^{\mathrm{S}}, \xi_{\mathrm{i}}{ }^{\mathrm{I}}, \xi_{\mathrm{i}}{ }^{\mathrm{S}}\right)$ são atualizados. Esses novos valores são utilizados para verificar se os critérios de parada (4.66) ou (4.67), apresentados na próxima subseção, são satisfeitos. Satisfeitos estes critérios, o método pára e temos a solução ótima para o nosso PPNL; caso contrário, o método entra em um novo ciclo interno e vai procurar por novas direções de busca, até conseguir atingir uma convergência. 


\subsubsection{O CICLO EXTERNO}

Apresentamos o ciclo externo onde ocorrem as atualizações dos parâmetros $s_{i}, \mu$ e $\beta$, bem como das estimativas dos multiplicadores de Lagrange $\left(\mathrm{v}_{\mathrm{k}}^{\mathrm{I}}, \mathrm{v}_{\mathrm{k}}^{\mathrm{S}}, \xi_{\mathrm{i}}^{\mathrm{I}}, \xi_{\mathrm{i}}^{\mathrm{S}}\right)$ e sua inicialização. Além disso, também é mostrado quais são os critérios de parada que devem ser satisfeitos para que o método atinja uma convergência satisfatória.

\subsubsection{OS PARÂMETROS $s_{i}$ E O PARÂMETRO $\mu$}

Os parâmetros shift, $\mathrm{s}_{\mathrm{i}}$, servem para relaxar a região factível para os limites superior e inferior em $\mathrm{x}_{\mathrm{i}}$ e $\mathrm{z}_{\mathrm{i}}$. Em nosso trabalho, todos os parâmetros shifts terão valor constante igual a 1.

O parâmetro de barreira, $\mu$, é atualizado por uma simples regra de redução:

$$
\mu^{\mathrm{L}+1}=\frac{\mu^{\mathrm{L}}}{\gamma}
$$

em que e $L$ é a indexação referente à iteração externa e $\gamma>1$ é um parâmetro preestabelecido usualmente como sendo $\gamma=2$ ou $\gamma=10$. O valor inicial, $\mu_{0}$, é um valor positivo arbitrário, geralmente é usado $\mu_{0}=10^{-1}$ ou $\mu_{0}=10^{-2}$.

\subsubsection{O PARÂMETRO $\beta$}

O parâmetro $\beta$ é a tolerância da aproximação com relação à fronteira da região factível. Em conseqüência disso, ele também pode ser interpretado como a tolerância da proximidade das assíntotas dos logaritmos dos termos de barreira modificada. A idéia envolvida é a de evitar a dificuldade de se calcular o logaritmo quando se está muito próximo de sua assíntota, pois o logaritmo tende ao infinito. Vassiliadis e Brooks (1998) atualizavam esse parâmetro através da seguinte relação: 


$$
\beta^{(\mathrm{L}+1)}=\min \left[1-\frac{1-\beta^{(\mathrm{L})}}{\gamma}, \beta_{\max }\right]
$$

em que $0<\beta_{\max }<1$.

Porém, Chen e Vassiliadis (2003) propuseram esse parâmetro constante, isto é, $\beta=0,9$, o qual é utilizado neste trabalho.

\subsubsection{OS MULTIPLICADORES DE LAGRANGE DAS RESTRIÇÕES DE DESIGUALDADE}

Para atualizar os multiplicadores de Lagrange das restrições de desigualdade, adotamos o esquema proposto por Breitfeld e Shanno (1994c). Para isso, consideramos o problema (4.2), somente com restrições de desigualdade com $\mathrm{g}_{\mathrm{i}}(\mathrm{x}, \mathrm{z})$ representando $x_{k}-\underline{x_{k}}, \overline{x_{k}}-x_{k}, \quad z_{i}-\underline{c_{i}}$ e $\overline{c_{i}}-z_{i}$, com $m=2 m_{c}+2 m_{x}$, denotado de maneira simplificada por:

$$
\begin{aligned}
& \text { Minimizar } \mathrm{f}(\mathrm{x}) \\
& \text { sujeito a : } \mathrm{g}_{\mathrm{i}}(\mathrm{x}, \mathrm{z}) \geq 0, \mathrm{i}=1, \ldots, \mathrm{m}_{\mathrm{c}}
\end{aligned}
$$

e construímos a função barreira modificada associada ao PPNL (4.43), como segue:

$$
\mathrm{FBM}=\mathrm{f}(\mathrm{x})-\mu \sum_{\mathrm{i}=1}^{\mathrm{m}} \lambda_{\mathrm{i}} \phi\left(\mathrm{g}_{\mathrm{i}}(\mathrm{x}, \mathrm{z})\right)
$$

onde:

$$
\phi\left(g_{i}(x, z)\right)=\ln \left(s_{i}+\frac{g_{i}(x, z)}{\mu}\right), i=1,2, \ldots, \text { m, se } g_{i}(x, z) \geq-\beta s_{i} \mu
$$

ou

$$
\phi\left(g_{i}(x, z)\right)=Q\left(g_{i}(x, z)\right), i=1,2, \ldots, m, \text { se } g_{i}(x, z)<-\beta s_{i} \mu
$$




$$
\mathrm{Q}\left(\mathrm{g}_{\mathrm{i}}(\mathrm{x}, \mathrm{z})\right)=\frac{1}{2} \mathrm{q}_{\mathrm{a}, \mathrm{i}}\left(\mathrm{g}_{\mathrm{i}}(\mathrm{x}, \mathrm{z})\right)^{2}+\mathrm{q}_{\mathrm{b}, \mathrm{i}} \mathrm{g}_{\mathrm{i}}(\mathrm{x}, \mathrm{z})+\mathrm{q}_{\mathrm{c}, \mathrm{i}}, \mathrm{i}=1,2, \ldots, \mathrm{m},
$$

e a função Lagrangiana:

$$
\mathrm{FLBM}=\mathrm{f}(\mathrm{x})-\sum_{\mathrm{i}=1}^{\mathrm{m}} \lambda_{\mathrm{i}} \mathrm{g}_{\mathrm{i}}(\mathrm{x}, \mathrm{z})
$$

A seguir, nós encontramos os respectivos gradientes das equações (4.44) e (4.45):

$$
\begin{aligned}
& \nabla F B M=\nabla f(x)-\sum_{i=1}^{m} \mu \lambda_{i} \phi^{\prime}\left(g_{i}(x, z)\right) \nabla g_{i}(x, z) \\
& \nabla F L B M=\nabla f(x)-\sum_{i=1}^{m} \lambda_{i} \nabla g_{i}(x, z)
\end{aligned}
$$

Seja $\mathrm{x}^{\mathrm{L}+1}$ um ponto que foi determinado no ciclo interno e que é usado para atualizar as estimativas dos multiplicadores de Lagrange. Aplicando as condições de otimalidade de $1^{\text {a }}$ ordem e substituindo $\mathrm{x}^{\mathrm{L}+1}$ em (4.46) com $\mu$ e $\lambda$, indexados com uma iteração de atraso (ou seja, estamos utilizando $\mathrm{x}^{\mathrm{L}+1}$ para atualizá-los), temos:

$$
\nabla f\left(x^{L+1}\right)=\sum_{i=1}^{m} \mu^{\mathrm{L}} \lambda_{i}^{\mathrm{L}} \phi^{\prime}\left(\mathrm{g}_{\mathrm{i}}\left(\mathrm{x}^{\mathrm{L}+1}, \mathrm{z}^{\mathrm{L}+1}\right)\right) \nabla \mathrm{g}_{\mathrm{i}}\left(\mathrm{x}^{\mathrm{L}+1}, \mathrm{z}^{\mathrm{L}+1}\right)
$$

Agora, aplicando as condições de otimalidade e substituindo $\mathrm{x}^{\mathrm{L}+1}$ em (4.47), mas após ter ocorrido a atualização de $\mu$ e $\lambda$, temos:

$$
\nabla f\left(x^{L+1}\right)=\sum_{i=1}^{m} \lambda_{i}^{L+1} \nabla g_{i}\left(x^{L+1}, z^{L+1}\right)
$$

Finalmente, comparando (4.48) e (4.49) e usando (4.26) para $\phi^{\prime}(\mathrm{g}(\mathrm{x}, \mathrm{z}))$, chegamos no seguinte esquema para atualizar as estimativas dos multiplicadores de Lagrange associados às restrições de desigualdade: 


$$
\lambda_{i}^{\mathrm{L}+1}=\mu^{\mathrm{L}} \lambda_{\mathrm{i}}^{\mathrm{L}}\left(\frac{1}{\mu^{\mathrm{L}} \mathrm{s}_{\mathrm{i}}+\mathrm{g}_{\mathrm{i}}(\mathrm{x}, \mathrm{z})}\right), \mathrm{i}=1,2, \ldots, \mathrm{m}, \text { se } \mathrm{g}_{\mathrm{i}}(\mathrm{x}, \mathrm{z}) \geq-\beta^{\mathrm{L}} \mathrm{s}_{\mathrm{i}} \mu^{\mathrm{L}}
$$

ou

$$
\lambda_{i}^{\mathrm{L}+1}=\mu^{\mathrm{L}} \lambda_{i}^{\mathrm{L}}\left(q_{i}^{\mathrm{a}, \mathrm{L}} \mathrm{g}_{\mathrm{i}}(\mathrm{x}, \mathrm{z})+\mathrm{q}_{\mathrm{i}}^{\mathrm{b}, \mathrm{L}}\right), \mathrm{i}=1,2, \ldots, \mathrm{m}, \text { se } \mathrm{g}_{\mathrm{i}}(\mathrm{x}, \mathrm{z})<-\beta^{\mathrm{L}} \mathrm{s}_{\mathrm{i}} \mu^{\mathrm{L}}
$$

em que $\lambda_{\mathrm{i}}$ agora representa todos os multiplicadores de Lagrange $\left(\mathrm{v}^{\mathrm{I}}, \mathrm{v}^{\mathrm{S}}, \xi^{\mathrm{I}}\right.$ e $\left.\xi^{\mathrm{S}}\right)$ para as restrições de desigualdade; $\mathrm{g}_{\mathrm{i}}(\mathrm{x}, \mathrm{z})$ representa todas as restrições de desigualdade e L é a indexação referente à iteração externa.

\subsection{INICIALIZAÇÃO DOS MULTIPLICADORES DE LAGRANGE}

Para problemas de otimização complexos, uma boa estimativa dos multiplicadores de Lagrange se faz necessária para obter a convergência do método da FLBMP. Neste trabalho, utilizamos o método da função Lagrangiana barreira clássica/penalidade (FLBCP) para a inicialização desses multiplicadores. Essa idéia foi proposta por Vassiliadis e Brooks (1998). Sua proposta é baseada no fato de que a FLBMP pode convergir em poucas iterações usando boas estimativas dos multiplicadores de Lagrange (teoricamente em 1 iteração com valores exatos - Polyak (1992)), e com valores de $\mu$ grandes (convergência antecipada), consequentemente preserva o bom condicionamento da matriz Hessiana.

Com o objetivo de determinarmos uma boa estimativa para os multiplicadores de Lagrange, nos baseamos no esquema de inicialização proposto por Fiacco e McCormick, (1968) e para isso, construímos a função barreira clássica logarítmica associada à (4.43):

$$
\mathrm{FBC}=\mathrm{f}(\mathrm{x})-\mu \sum_{\mathrm{i}=1}^{\mathrm{m}} \phi_{\mathrm{FBC}}\left(\mathrm{g}_{\mathrm{i}}(\mathrm{x}, \mathrm{z})\right)=\mathrm{f}(\mathrm{x})-\mu \sum_{\mathrm{i}=1}^{\mathrm{m}} \ln \left(\mathrm{g}_{\mathrm{i}}(\mathrm{x}, \mathrm{z})\right)
$$


Consideramos, também, a função Lagrangiana associada à (4.43):

$$
\text { FLBC }=f(x)-\sum_{i=1}^{m} \lambda_{i} g_{i}(x, z)
$$

Determinamos os respectivos gradientes das funções (4.52) e (4.53):

$$
\begin{aligned}
& \nabla F B C=\nabla f(x)-\sum_{i=1}^{m} \frac{\mu}{g_{i}(x, z)} \nabla g_{i}(x, z) \\
& \nabla F L B C=\nabla f(x)-\sum_{i=1}^{m} \lambda_{i} \nabla g_{i}(x, z)
\end{aligned}
$$

Comparando (4.54) e (4.55), deduzimos a seguinte estimativa para $\lambda$ :

$$
\lambda^{\text {estimado }}=\frac{\mu^{\mathrm{L}}}{\mathrm{g}_{\mathrm{i}}(\mathrm{x}, \mathrm{z})}
$$

onde x é o valor encontrado pelo estágio externo do método da FBC. A fórmula (4.56) fornece uma estimativa para os multiplicadores de Lagrange $\left(v^{\mathrm{I}}, \mathrm{v}^{\mathrm{S}}, \xi^{\mathrm{I}}\right.$ e $\left.\xi^{\mathrm{S}}\right)$ baseada na solução encontrada pelo método da função barreira clássica logarítmica (FBC).

\subsection{ESQUEMA DE EXTRAPOLAÇÃO DA FBC}

Como no método FLBMP, um esquema de extrapolação é introduzido no método FBC, determinando a função Lagrangiana barreira clássica/penalidade, FLBCP. Deste modo, o método da FLBCP fornece os valores utilizados no cálculo das estimativas dos multiplicadores de Lagrange $\lambda$ (4.56). Com esse objetivo, construímos, inicialmente, a Função Barreira Clássica/Penalidade (FBCP) para o PPNL (4.2), obtendo assim, um novo PPNL equivalente a ele: 


$$
\begin{aligned}
\underset{\mathrm{x}, \mathrm{z}}{\operatorname{minimizar} \mathrm{FLBCP}=} & \mathrm{f}(\mathrm{x})-\mu \sum_{\mathrm{k}=1}^{\mathrm{m}_{\mathrm{x}}}\left[\phi_{\mathrm{FBC}}\left(\mathrm{x}_{\mathrm{k}}-\underline{\mathrm{x}_{\mathrm{k}}}\right)+\phi_{\mathrm{FBC}}\left(\overline{\mathrm{x}_{\mathrm{k}}}-\mathrm{x}_{\mathrm{k}}\right)\right]- \\
& -\mu \sum_{\mathrm{i}=1}^{\mathrm{m}_{\mathrm{C}}}\left[\phi_{\mathrm{FBC}}\left(\mathrm{z}_{\mathrm{i}}-\underline{\mathrm{C}_{\mathrm{i}}}\right)+\phi_{\mathrm{FBC}}\left(\overline{\mathrm{c}_{\mathrm{i}}}-\mathrm{z}_{\mathrm{i}}\right)\right]
\end{aligned}
$$

sujeito a: $c_{i}(x)-z_{i}=0, i=1,2, \ldots, m_{c}$

$$
h_{j}(x)=0, j=1,2, \ldots, m_{h} \text {, }
$$

e, como conseqüência determinamos a função Lagrangiana associada ao PPNL (4.57) é dada por:

$$
\begin{aligned}
\text { FLBCP }= & f(\mathrm{x})-\mu \sum_{\mathrm{k}=1}^{\mathrm{m}_{\mathrm{x}}}\left[\phi_{\mathrm{FBC}}\left(\mathrm{x}_{\mathrm{k}}-\underline{\mathrm{x}_{\mathrm{k}}}\right)+\phi_{\mathrm{FBC}}\left(\overline{\mathrm{x}_{\mathrm{k}}}-\mathrm{x}_{\mathrm{k}}\right)\right]- \\
& -\mu \sum_{\mathrm{i}=1}^{\mathrm{m}_{\mathrm{C}}}\left[\phi_{\mathrm{FBC}}\left(\mathrm{z}_{\mathrm{i}}-\underline{\mathrm{c}_{\mathrm{i}}}\right)+\phi_{\mathrm{FBC}}\left(\overline{\mathrm{c}_{\mathrm{i}}}-\mathrm{z}_{\mathrm{i}}\right)\right]+\sum_{\mathrm{j}=1}^{\mathrm{m}_{\mathrm{h}}} \lambda_{\mathrm{j}}^{\mathrm{E}} \mathrm{h}_{\mathrm{j}}(\mathrm{x})+\sum_{\mathrm{i}=1}^{\mathrm{m}_{\mathrm{c}}} \lambda_{\mathrm{i}}^{\mathrm{I}}\left(\mathrm{c}_{\mathrm{i}}(\mathrm{x})-\mathrm{z}_{\mathrm{i}}\right)
\end{aligned}
$$

em que a função Lagrangeana será minimizada com respeito às variáveis primais, x e z, e a função $\phi_{\mathrm{FBC}}$ é definida da seguinte forma:

$$
\left\{\begin{array}{l}
\phi_{F B C}\left(g_{i}(x, z)\right)=\ln \left(g_{i}(x, z)\right), i=1,2, \ldots, m, \text { se } g_{i}(x, z) \geq(1-\beta) s_{i} \mu \\
\phi_{F B C}\left(g_{i}(x, z)\right)=Q_{F B C}\left(g_{i}(x, z)\right), i=1,2, \ldots, m, \text { se } g_{i}(x, z)<(1-\beta) s_{i} \mu
\end{array}\right.
$$

com:

$$
\mathrm{Q}_{\mathrm{FBC}}\left(\mathrm{g}_{\mathrm{i}}(\mathrm{x}, \mathrm{z})\right)=\frac{1}{2} \mathrm{a}_{\mathrm{i}}\left(\mathrm{g}_{\mathrm{i}}(\mathrm{x}, \mathrm{z})\right)^{2}+\mathrm{b}_{\mathrm{i}} \mathrm{g}_{\mathrm{i}}(\mathrm{x}, \mathrm{z})+\mathrm{c}_{\mathrm{i}},
$$

sendo uma função extrapolação quadrática.

A função é construída de tal forma que o seu valor, bem como os das suas derivadas de primeira e segunda ordem coincidam com os da função barreira logarítmica $B_{c}(g(x, z))=\ln (g(x, z))$ no ponto $g(x, z)=(1-\beta) s \mu$. Logo, temos as seguintes igualdades: 


$$
\begin{aligned}
& \mathrm{Q}(\mathrm{g}(\mathrm{x}, \mathrm{z}))=\mathrm{B}_{\mathrm{c}}(\mathrm{g}(\mathrm{x}, \mathrm{z})) \text {, isto é, } \frac{1}{2} \mathrm{a}(\mathrm{g}(\mathrm{x}, \mathrm{z}))^{2}+\mathrm{bg}(\mathrm{x}, \mathrm{z})+\mathrm{c}=\ln (\mathrm{g}(\mathrm{x}, \mathrm{z})) \\
& \mathrm{Q}^{\prime}(\mathrm{g}(\mathrm{x}, \mathrm{z}))=\mathrm{B}_{\mathrm{c}}{ }^{\prime}(\mathrm{g}(\mathrm{x}, \mathrm{z})) \text {, isto é, } \mathrm{ag}(\mathrm{x}, \mathrm{z})+\mathrm{b}=\frac{1}{\mathrm{~g}(\mathrm{x}, \mathrm{z})} \\
& \mathrm{Q}^{\prime \prime}(\mathrm{g}(\mathrm{x}, \mathrm{z}))=\mathrm{B}_{\mathrm{c}}{ }^{\prime \prime}(\mathrm{g}(\mathrm{x}, \mathrm{z})) \text {, isto é, } \mathrm{a}=-\frac{1}{\mathrm{~g}(\mathrm{x}, \mathrm{z})^{2}}
\end{aligned}
$$

Substituindo $g(x, z)=(1-\beta) s \mu$ em (4.63), temos:

$$
\mathrm{a}=-\frac{1}{\mathrm{~g}(\mathrm{x}, \mathrm{z})^{2}}=-\frac{1}{((1-\beta) s \mu)^{2}}
$$

Substituindo $g(x, z)=(1-\beta) s \mu$ e (4.64) em (4.62), temos:

$$
\begin{aligned}
& -\frac{1}{((1-\beta) s \mu)^{2}}((1-\beta) s \mu)+b=\frac{1}{(1-\beta) s \mu}, \text { logo, } \\
& b=\frac{2}{((1-\beta) s \mu)}
\end{aligned}
$$

Substituindo $g(x, z)=(1-\beta) s \mu,(4.64)$ e (4.65) em (4.61), temos:

$$
\begin{aligned}
& \frac{1}{2}\left(-\frac{1}{((1-\beta) s \mu)^{2}}\right)((1-\beta) s \mu)^{2}+\left(\frac{2}{(1-\beta) s \mu}\right)((1-\beta) s \mu)+c=\ln ((1-\beta) s \mu), \text { ou } \\
& -\frac{1}{2}+2+c=\ln ((1-\beta) s \mu) . \text { Isolando c, temos: } \\
& c=\ln ((1-\beta) s \mu)-\frac{3}{2}
\end{aligned}
$$

Portanto, as equações (4.64), (4.65) e (4.66) fornecem os coeficientes da função extrapolação quadrática (4.60) para a FLBCP, ou seja,

$$
a_{i}=-\frac{1}{\left((1-\beta) s_{i} \mu\right)^{2}}, b_{i}=\frac{2}{(1-\beta) s_{i} \mu} \text { e }_{i}=\ln \left((1-\beta) s_{i} \mu\right)-\frac{3}{2}
$$


Dessa forma, a FLBCP que utilizamos é uma função a qual relaxa a região factível do problema (4.2). Fixamos o número de iterações do método FLBCP em uma ou duas e obtemos valores estimados para as variáveis primais e duais. Esses valores serão utilizados no cálculo das estimativas dos multiplicadores de Lagrange, (4.56). O método FLBMP será então iniciado tendo esses valores como solução inicial.

\subsection{MÉTODO DE NEWTON PARA A FLBCP}

Da mesma forma que no método da FLBMP, aplicamos as condições de otimalidade de $1^{\mathrm{a}}$ ordem na FLBCP (4.58), obtemos um sistema não-linear, como segue:

$$
\left\{\begin{array}{l}
\frac{\partial \mathrm{FLBCP}}{\partial \mathrm{x}}=0 \\
\frac{\partial \mathrm{FLBCP}}{\partial \mathrm{z}}=0 \\
\frac{\partial \mathrm{FLBCP}}{\partial \lambda^{\mathrm{E}}}=0 \\
\frac{\partial \mathrm{FLBCP}}{\partial \lambda^{\mathrm{I}}}=0
\end{array}\right.
$$

Determinamos a solução do sistema não-linear (4.68) utilizando o método de Newton. A aplicação do método de Newton gera as direções de busca, sx, sz, s $\lambda^{\mathrm{E}}$ e $s \lambda^{\mathrm{I}}$, as quais são utilizadas para a atualização das variáveis do sistema e resulta no sistema matricial, que, em sua forma simplificada, é representado por:

$$
\mathrm{H}_{\mathrm{FLBCP}} \mathrm{S}=-\mathrm{G}_{\mathrm{FLBCP}}
$$

em que $\mathrm{G}_{\mathrm{FLBCP}}$ e $\mathrm{H}_{\mathrm{FLBCP}}$ são, respectivamente, o gradiente e a Hessiana da FLBCP (4.58) e $\mathrm{S}=\left(\mathrm{sx}, \mathrm{sz}, \mathrm{s} \lambda^{\mathrm{E}}\right.$, s $\left.\lambda^{\mathrm{I}}\right)$ é o vetor direção de busca.

Análogo ao método FLBMP, G à (4.25), trocando $G_{F L B M P}$ por $G_{F L B C P}$ e a FLBMP pela FLBCP. 
Ao diferenciar os termos de barreira clássica/penalidade produzimos, neste caso, uma fórmula algébrica geral:

$$
\left\{\begin{array}{l}
\phi^{\prime}\left(g_{i}(x, z)\right)=\frac{1}{g_{i}(x, z)}, i=1,2, \ldots, \text { m, se } g_{i}(x, z) \geq(1-\beta) s_{i} \mu \\
\phi^{\prime}\left(g_{i}(x, z)\right)=a_{i} g_{i}(x, z)+b_{i}, i=1,2, \ldots, \text { m, se } g_{i}(x, z)<(1-\beta) s_{i} \mu
\end{array}\right.
$$

A Hessiana da FLBCP toma a mesma forma da apresentada de (4.27) à (4.29), trocando $\mathrm{H}_{\text {FLBMP }}$ por $\mathrm{H}_{\mathrm{FLBCP}}$ e a FLBMP pela FLBCP. E definimos uma fórmula algébrica geral para as derivadas de $2^{\mathrm{a}}$ ordem dos termos de barreira modificada/penalidade:

$$
\left\{\begin{array}{l}
\phi^{\prime \prime}\left(g_{i}(x, z)\right)=-\frac{1}{\left(g_{i}(x, z)\right)^{2}}, i=1,2, \ldots, \text { m, se } g_{i}(x, z) \geq(1-\beta) s_{i} \mu \\
\phi^{\prime \prime}\left(g_{i}(x, z)\right)=a_{i}, i=1,2, \ldots, \text { m, se } g_{i}(x, z)<(1-\beta) s_{i} \mu
\end{array}\right.
$$

Para resolver o sistema matricial (4.69) utilizamos o mesmo esquema mostrado na subseção 4.3.7, bem como a busca linear e a forma de atualizar as variáveis primais e duais.

A função penalidade mérito, no caso da FLBCP, toma a seguinte forma:

$$
\begin{aligned}
\mathrm{FPM}_{\mathrm{b}}= & \mathrm{f}(\mathrm{x})-\mu \sum_{\mathrm{k}=1}^{\mathrm{m}_{\mathrm{x}}}\left[\phi_{\mathrm{FBC}}\left(\mathrm{x}_{\mathrm{k}}-\underline{\mathrm{x}_{\mathrm{k}}}\right)+\phi_{\mathrm{FBC}}\left(\overline{\mathrm{x}_{\mathrm{k}}}-\mathrm{x}_{\mathrm{k}}\right)\right]-\mu \sum_{\mathrm{i}=1}^{\mathrm{m}_{\mathrm{C}}}\left[\phi_{\mathrm{FBC}}\left(\mathrm{z}_{\mathrm{i}}-\underline{\mathrm{c}_{\mathrm{i}}}\right)+\phi_{\mathrm{FBC}}\left(\overline{\mathrm{c}_{\mathrm{i}}}-\mathrm{z}_{\mathrm{i}}\right)\right]+ \\
& +\sum_{\mathrm{j}=1}^{\mathrm{m}_{\mathrm{h}}} \max \left[\mathrm{M}_{\mathrm{j}},\left|\lambda_{\mathrm{j}}^{\mathrm{E}}\right|\right]\left|\cdot \mathrm{h}_{\mathrm{j}}(\mathrm{x})\right|+\sum_{\mathrm{i}=1}^{\mathrm{m}_{\mathrm{c}}} \max \left[\mathrm{M}_{\mathrm{i}},\left|\lambda_{\mathrm{i}}^{\mathrm{I}}\right|\right]|\cdot| \mathrm{c}_{\mathrm{i}}(\mathrm{x})-\mathrm{z}_{\mathrm{i}} \mid
\end{aligned}
$$

Com isso, aplicamos uma ou duas iterações do método da FLBCP e temos uma solução primal/dual inicial para o método da FLBMP, e as estimativas dos multiplicadores de Lagrange relacionados às restrições de desigualdade da FLBMP usando (4.56). 


\subsubsection{CRITÉRIOS DE PARADA PARA O CICLO EXTERNO}

O ciclo externo do método da FLBMP pode ser considerado completamente convergido através da satisfação de cada uma das seguintes condições:

$$
\mathrm{u}_{1}^{\mathrm{k}}<0
$$

ou

$$
\mathrm{u}_{2}{ }^{\mathrm{k}}<\tau \mathrm{e} \mathrm{u}_{3}{ }^{\mathrm{k}}<0
$$

onde $\mathrm{u}_{1}{ }^{\mathrm{k}}, \mathrm{u}_{2}{ }^{\mathrm{k}}$ e $\mathrm{u}_{3}{ }^{\mathrm{k}}$ são calculados como indicados na Tabela 1 , seguindo as diretrizes de GILL et al. (1981). Lembramos que, $\mathrm{g}_{\mathrm{i}}(\mathrm{x}, \mathrm{z})$ representa as restrições de desigualdade $\left(\mathrm{x}_{\mathrm{k}}-\underline{\mathrm{x}_{\mathrm{k}}}, \overline{\mathrm{x}_{\mathrm{k}}}-\mathrm{x}_{\mathrm{k}}, \mathrm{z}_{\mathrm{i}}-\underline{\mathrm{c}_{\mathrm{i}}}\right.$ e $\left.\overline{\mathrm{c}_{\mathrm{i}}}-\mathrm{z}_{\mathrm{i}}\right)$ enquanto $\lambda_{\mathrm{i}}$ representa os Multiplicadores de Lagrange dessas restrições limite $\left(\xi^{\mathrm{I}}, \xi^{\mathrm{S}}, \mathrm{v}_{\mathrm{i}}^{\mathrm{I}}\right.$ e $\left.\mathrm{v}_{\mathrm{i}}^{\mathrm{S}}\right)$.

TABELA 1 - Critérios de parada

\begin{tabular}{|l|l|}
\hline Parâmetro & Comentário \\
\hline $\mathrm{u}_{1}^{\mathrm{k}}=\max \left[\mathrm{A}^{\mathrm{k}}-\eta, \mathrm{B}^{\mathrm{k}}-\eta, \Gamma^{\mathrm{k}}-(\eta)^{\frac{1}{3}}, \Delta^{\mathrm{k}}-(\eta)^{\frac{1}{3}}\right]$ & $\begin{array}{l}\text { Critério de convergência global } \\
\text { desejável. }\end{array}$ \\
\hline $\mathrm{u}_{2}^{\mathrm{k}}=\max \left[\mathrm{A}^{\mathrm{k}}, \Gamma^{\mathrm{k}}\right]$ & $\begin{array}{l}\text { Satisfaz a factibilidade e a } \\
\text { complementaridade }\end{array}$ \\
\hline $\mathrm{u}_{3}^{\mathrm{k}}=\max \left[\mathrm{E}^{\mathrm{k}}-(\eta / 10), \mathrm{Z}^{\mathrm{k}}-(\eta)^{\frac{1}{2}}\right]$ & Baixa medida de convergência \\
\hline $\mathrm{A}^{\mathrm{k}}=\max _{\mathrm{i}}\left[\min \left[\mathrm{g}_{\mathrm{i}}(\mathrm{x}, \mathrm{z}), 0\right]\right]$ & Factibilidade das inequações \\
\hline $\left.\mathrm{B}^{\mathrm{k}}=\max _{\mathrm{i}}\left|\lambda_{\mathrm{i}} \mathrm{g}_{\mathrm{i}}(\mathrm{x})\right| /\left(1+\mid \mathrm{f}\left(\mathrm{x}^{\mathrm{k}}\right)\right)\right]$ & Complementaridade escalar \\
\hline$\left.\Gamma^{\mathrm{k}}=\left\|\nabla_{\mathrm{x}, \mathrm{z}, \lambda^{\mathrm{u}}, \lambda^{2}} \mathrm{~L}\left(\mathrm{x}^{\mathrm{k}}, \mathrm{z}^{\mathrm{k}}, \mu^{\mathrm{k}}, \mathrm{v}^{\mathrm{k}}, \xi^{\mathrm{k}}\right)\right\|_{\infty} /\left(1+\mid \mathrm{f}\left(\mathrm{x}^{\mathrm{k}}\right)\right)\right)$ & $\begin{array}{l}\text { Norma escalar do Gradiente da } \\
\text { Lagrangiana }\end{array}$ \\
\hline$\Delta^{\mathrm{k}}=\left\|\nabla_{\mathrm{x}} \mathrm{f}\left(\mathrm{x}^{\mathrm{k}}\right)+\sum_{\mathrm{i}=1}^{\mathrm{m}} \lambda_{\mathrm{i}} \nabla_{\mathrm{x}} \mathrm{g}_{\mathrm{i}}(\mathrm{x})\right\|_{\infty} /\left(1+\mid \mathrm{f}\left(\mathrm{x}^{\mathrm{k}}\right)\right)$ & $\begin{array}{l}\text { Condições de } 1^{\mathrm{a}} \text { ordem escalar para } \\
\text { problemas restritos }\end{array}$ \\
\hline $\mathrm{E}^{\mathrm{k}}=\mid \mathrm{f}\left(\mathrm{x}^{\mathrm{k}}\right)-\mathrm{f}\left(\mathrm{x}^{\mathrm{k}-1}\right) / /\left(1+\mid \mathrm{f}\left(\mathrm{x}^{\mathrm{k}}\right)\right)$ & Mudança suficiente no objetivo \\
\hline
\end{tabular}




\begin{tabular}{|l|l|}
\hline $\mathrm{Z}^{\mathrm{k}}=\left\|\left(\mathrm{x}^{\mathrm{k}}\right)^{\mathrm{T}}-\left(\mathrm{x}^{\mathrm{k}-1}\right)^{\mathrm{T}}\right\|_{2}$ & $\begin{array}{l}\text { Mudança suficiente no vetor variável } \\
\text { primal }\end{array}$ \\
\hline$\eta$ & $\begin{array}{l}\text { Tolerância usual especificada. Pode } \\
\text { ser } 10^{-5} .\end{array}$ \\
\hline
\end{tabular}

\subsubsection{ALGORITMO DO MÉTODO DA FLBMP}

A fim de sintetizar todos os passos envolvidos no método da FLBMP, propomos dois algoritmos básicos envolvidos com a abordagem proposta: algoritmo da inicialização com a FLBCP e algoritmo da FLBMP.

Primeiramente mostraremos um algoritmo para o método da FLBCP o qual é utilizado para determinar uma solução inicial para o método da FLBMP. Logo em seguida, será mostrado um algoritmo para o método da FLBMP, que é utilizado para encontrar a solução ótima do problema de otimização (4.1).

\subsubsection{ALGORITMO DA INICIALIZAÇÃO COM A FLBCP}

\section{Passo inicial}

Dado o problema (4.1) construa a FLBCP conforme (4.58);

Faça $K=0$;

Escolha uma solução inicial para as variáveis do problema: $x^{0}, z^{0},\left(\lambda^{E}\right)^{0}$ e $\left(\lambda^{I}\right)^{0}$.

\section{$\underline{\text { Passo iterativo }}$}

i) Determine o sistema para a FLBCP análogo ao sistema (4.68) e resolva-o;

ii) Atualize as variáveis: $x^{0}$ e $z^{0}$ utilizando (4.35), $\left(\lambda^{\mathrm{E}}\right)^{0}$ e $\left(\lambda^{\mathrm{I}}\right)^{0}$ utilizando (4.36) e (4.37);

iii) Se o critério de parada para o método de Newton está satisfeito, vá ao passo iv;

Senão, volte ao passo i; 
iv) Se as variáveis do problema satisfazem as condições de KKT ou se $\mathrm{K}>2$, calcule as estimativas dos multiplicadores de Lagrange $\left(\mathrm{v}^{\mathrm{I}}, \mathrm{v}^{\mathrm{S}}\right.$, $\xi^{\mathrm{I}}$ e $\left.\xi^{\mathrm{S}}\right)$ utilizando (4.56) e FIM;

Senão, vá ao passo v;

v) Atualize o fator barreira utilizando (4.41), faça $K=K+1$ e volte a i.

\subsubsection{ALGORITMO DA FLBMP}

\section{$\underline{\text { Passo inicial }}$}

Inicie as variáveis com o algoritmo 4.3.3.1;

Dado o problema (4.1) construa a FLBMP (4.17);

Faça $\mathrm{K}=0$;

\section{$\underline{\text { Passo iterativo }}$}

i) Determine o sistema (3.19) e resolva-o;

ii) Atualize as variáveis: $x^{0}$ e $z^{0}$ utilizando (4.35) e $\left(\lambda^{\mathrm{E}}\right)^{0}$ e $\left(\lambda^{\mathrm{I}}\right)^{0}$ utilizando (4.36) e (4.37);

iii) Se o critério de parada para o método de Newton está satisfeito, vá ao passo iv;

Senão, volte ao passo i;

iv) Se as variáveis do problema satisfazem as condições de KKT, FIM.

Senão, vá ao passo v;

v) Atualize o fator barreira utilizando (4.41) e os multiplicadores de Lagrange $\left(v^{\mathrm{I}}, \mathrm{v}^{\mathrm{S}}, \xi^{\mathrm{I}}\right.$ e $\left.\xi^{\mathrm{S}}\right)$ utilizando (4.50) ou (4.51), faça $\mathrm{K}=\mathrm{K}+1$ e volte a $\mathbf{i}$.

As figuras 1 e 2 mostram, de forma simplificada, o funcionamento dos ciclos interno e externo do algoritmo do método da FLBMP inicializado com o método da FLBCP. 


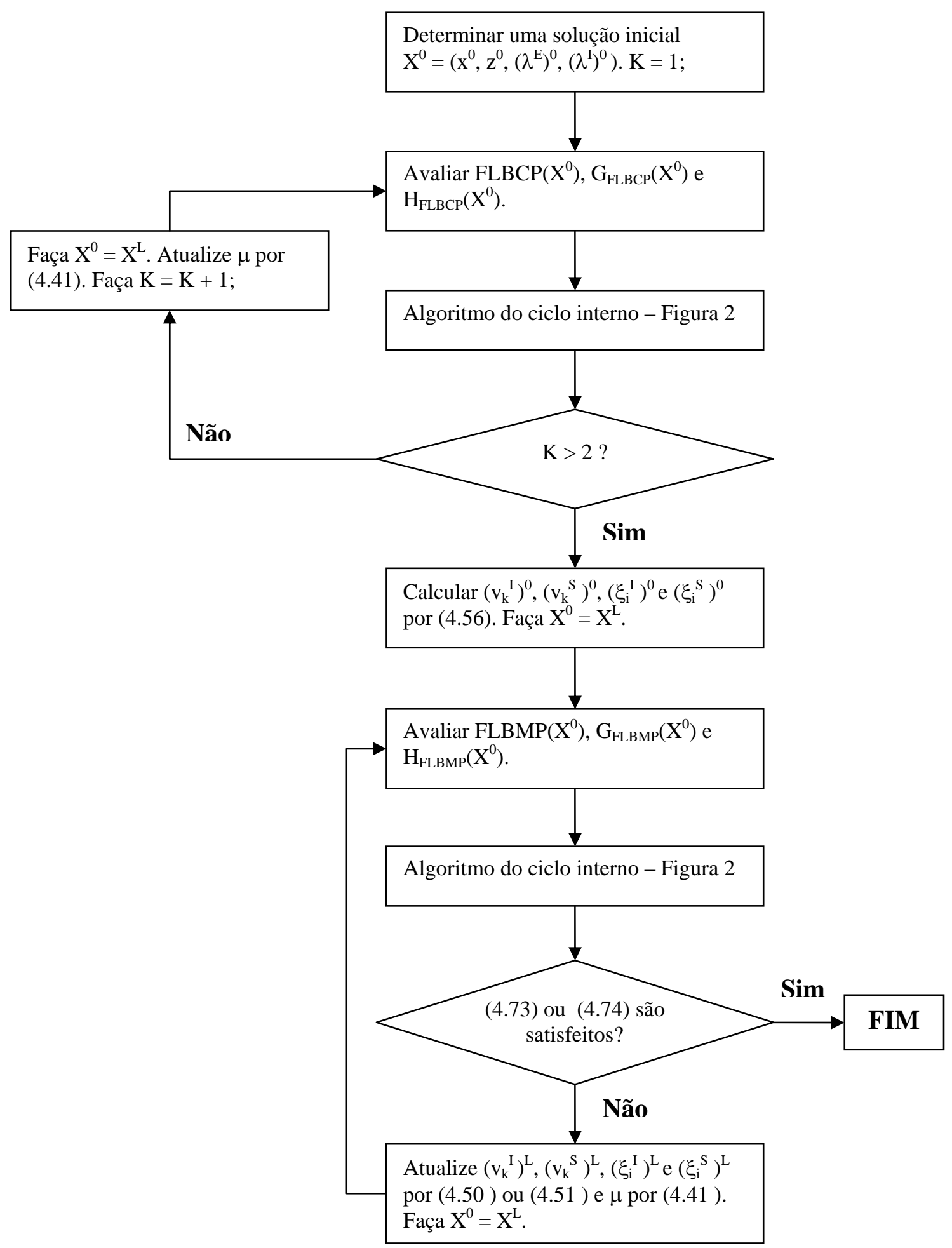

FIGURA 1: Ciclo externo do algoritmo do método da FLBMP com inicialização pelo método da FLBCP. 


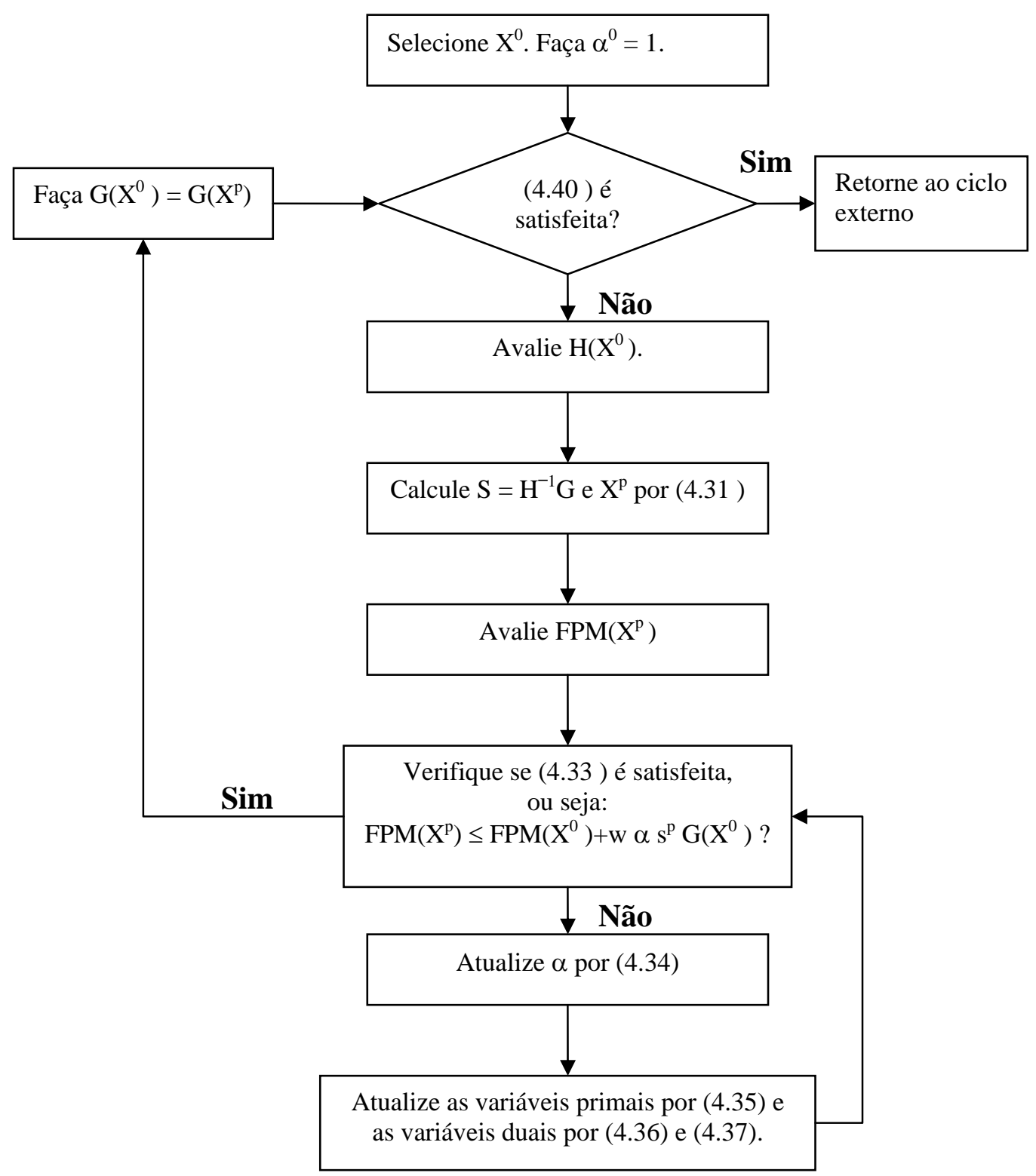

FIGURA 2: Ciclo interno dos métodos da FLBCP e da FLBMP.

A figura 2 mostra, de forma simplificada, o ciclo interno para os métodos da FLBCP e FLBMP. Por esta razão, o vetor gradiente está denotado por G e a matriz Hessiana por H. Encontram-se no Apêndice 1 os algoritmos estruturados.

No próximo capítulo apresentamos OS resultados computacionais da aplicação do método da FLBMP em um problema teórico e em problemas de FPO. 


\section{CAPÍTULO 5}

\section{RESULTADOS NUMÉRICOS}

Neste capítulo apresentamos os resultados numéricos obtidos através da aplicação do método da função Lagrangiana barreira modificada/penalidade com a finalidade de demonstrar seu desempenho.

\subsection{EXEMPLO 1}

Considere o seguinte problema:

$$
\begin{array}{ll}
\text { Minimizar } & \left(\mathrm{x}_{1}-2\right)^{4}+\left(\mathrm{x}_{1}-2 \mathrm{x}_{2}\right)^{2} \\
\text { sujeito a: } & \mathrm{x}_{1}+\mathrm{x}_{2}-3=0 \\
& -1 \leq \mathrm{x}_{1}^{2}-\mathrm{x}_{2} \leq 0 \\
& 1,5 \leq \mathrm{x}_{2} \leq 2
\end{array}
$$




\subsubsection{INICIALIZAÇÃO DO MÉTODO USANDO A FLBCP}

Para a fase de inicialização, associada ao problema (5.1) temos a função Lagrangiana barreira clássica penalidade:

$$
\begin{aligned}
\mathrm{FLBCP}= & \left(\mathrm{x}_{1}-2\right)^{4}+\left(\mathrm{x}_{1}-2 \mathrm{x}_{2}\right)^{2}-\mu\left[\phi_{\mathrm{FBC}}\left(\mathrm{x}_{2}-1,5\right)+\phi_{\mathrm{FBC}}\left(2-\mathrm{x}_{2}\right)+\phi_{\mathrm{FBC}}(\mathrm{z}+1)+\right. \\
& \left.+\phi_{\mathrm{FBC}}(-\mathrm{z})\right]+\lambda^{\mathrm{E}}\left(\mathrm{x}_{1}+\mathrm{x}_{2}-3\right)+\lambda^{\mathrm{I}}\left(\mathrm{x}_{1}^{2}-\mathrm{x}_{2}-\mathrm{z}\right)
\end{aligned}
$$

em que:

$$
\begin{aligned}
& \phi_{\mathrm{FBC}}\left(\mathrm{x}_{2}-1,5\right)=\left\{\begin{array}{c}
\ln \left(\mathrm{x}_{2}-1,5\right), \text { se } \mathrm{x}_{2}-1,5 \geq(1-\beta) \mathrm{s} \mu \\
\frac{1}{2} \mathrm{a}\left(\mathrm{x}_{2}-1,5\right)^{2}+\mathrm{b}\left(\mathrm{x}_{2}-1,5\right)+\mathrm{c}, \text { se } \mathrm{x}_{2}-1,5<(1-\beta) \mathrm{s} \mu
\end{array}\right. \\
& \phi_{\mathrm{FBC}}\left(2-\mathrm{x}_{2}\right)=\left\{\begin{array}{c}
\ln \left(2-\mathrm{x}_{2}\right), \text { se } 2-\mathrm{x}_{2} \geq(1-\beta) \mathrm{s} \mu \\
\frac{1}{2} \mathrm{a}\left(2-\mathrm{x}_{2}\right)^{2}+\mathrm{b}\left(2-\mathrm{x}_{2}\right)+\mathrm{c}, \text { se } 2-\mathrm{x}_{2}<(1-\beta) \mathrm{s} \mu
\end{array} ;\right. \\
& \phi_{\mathrm{FBC}}(\mathrm{z}+1)=\left\{\begin{array}{c}
\ln (\mathrm{z}+1), \text { se } \mathrm{z}+1 \geq(1-\beta) \mathrm{s} \mu \\
\frac{1}{2} \mathrm{a}(\mathrm{z}+1)^{2}+\mathrm{b}(\mathrm{z}+1)+\mathrm{c}, \mathrm{se} \mathrm{z}+1<(1-\beta) \mathrm{s} \mu
\end{array} ;\right. \\
& \phi_{\mathrm{FBC}}(-\mathrm{z})=\left\{\begin{array}{c}
\ln (-\mathrm{z}), \mathrm{se}-\mathrm{z} \geq(1-\beta) \mathrm{s} \mu \\
\frac{1}{2} \mathrm{a}(-\mathrm{z})^{2}+\mathrm{b}(-\mathrm{z})+\mathrm{c} \mathrm{se}-\mathrm{z}<(1-\beta) \mathrm{s} \mu
\end{array} ;\right. \\
& \mathrm{a}=-\frac{1}{((1-\beta) \mathrm{s} \mu)^{2}} ; \mathrm{b}=\frac{2}{(1-\beta) s \mu} ; \mathrm{e} \mathrm{c}=\ln ((1-\beta) \mathrm{s} \mu)-\frac{3}{2}
\end{aligned}
$$

Aplicando as condições de otimalidade à função (5.2) obtemos um sistema de equações não-lineares, cuja solução pode ser determinada pelo método de Newton gerando o seguinte sistema linear:

$$
\mathrm{H}_{\mathrm{FLBCP}} \mathrm{S}=-\mathrm{G}_{\mathrm{FLBCP}}
$$


em que:

$$
\mathrm{G}_{\mathrm{FLBCP}}=\left[\begin{array}{l}
\frac{\partial \mathrm{FLBCP}}{\partial \mathrm{x}_{1}} \\
\frac{\partial \mathrm{FLBCP}}{\partial \mathrm{x}_{2}} \\
\frac{\partial \mathrm{FLBCP}}{\partial \mathrm{z}} \\
\frac{\partial \mathrm{FLBCP}}{\partial \lambda^{\mathrm{E}}} \\
\frac{\partial \mathrm{FLBCP}}{\partial \lambda^{\mathrm{I}}}
\end{array}\right]
$$

é o vetor gradiente com

$$
\begin{aligned}
& \frac{\partial \text { FLBCP }}{\partial \mathrm{x}_{1}}=4\left(\mathrm{x}_{1}-2\right)^{3}+2\left(\mathrm{x}_{1}-2 \mathrm{x}_{2}\right)+\lambda^{\mathrm{E}}+2 \mathrm{x}_{1} \lambda^{\mathrm{I}} \\
& \frac{\partial \mathrm{FLBCP}}{\partial \mathrm{x}_{2}}=-4\left(\mathrm{x}_{1}-2 \mathrm{x}_{2}\right)-\mu\left[\phi_{\mathrm{FBC}}^{\prime}\left(\mathrm{x}_{2}-1,5\right)-\phi_{\mathrm{FBC}}^{\prime}\left(2-\mathrm{x}_{2}\right)\right]+\lambda^{\mathrm{E}}-\lambda^{\mathrm{I}} ; \\
& \frac{\partial \mathrm{FLBCP}}{\partial \mathrm{z}}=-\mu\left[\phi_{\mathrm{FBC}}^{\prime}(\mathrm{z}+1)-\phi_{\mathrm{FBC}}^{\prime}(-\mathrm{z})\right]-\lambda^{\mathrm{I}} \text {; } \\
& \frac{\partial \mathrm{FLBCP}}{\partial \lambda^{\mathrm{E}}}=\mathrm{x}_{1}+\mathrm{x}_{2}-3 \\
& \frac{\partial \mathrm{FLBCP}}{\partial \lambda^{\mathrm{I}}}=\mathrm{x}_{1}^{2}-\mathrm{x}_{2}-\mathrm{z} \\
& \phi^{\prime}{ }_{\mathrm{FBC}}\left(\mathrm{x}_{2}-1,5\right)=\left\{\begin{array}{c}
\frac{1}{\mathrm{x}_{2}-1,5}, \text { se } \mathrm{x}_{2}-1,5 \geq-\beta \mathrm{s} \mu \\
\mathrm{a}\left(\mathrm{x}_{2}-1,5\right)+\mathrm{b}, \text { se } \mathrm{x}_{2}-1,5<-\beta \mathrm{s} \mu
\end{array}\right. \text {; } \\
& \phi_{\mathrm{FBC}}\left(2-\mathrm{x}_{2}\right)=\left\{\begin{array}{c}
\frac{1}{2-\mathrm{x}_{2}}, \text { se } 2-\mathrm{x}_{2} \geq(1-\beta) \mathrm{s} \mu \\
\mathrm{a}\left(2-\mathrm{x}_{2}\right)+\mathrm{b}, \text { se } 2-\mathrm{x}_{2}<(1-\beta) \mathrm{s} \mu
\end{array}\right. \text {; }
\end{aligned}
$$




$$
\begin{aligned}
& \phi^{\prime}{ }_{\mathrm{FBC}}(\mathrm{z}+1)=\left\{\begin{array}{c}
\frac{1}{\mathrm{z}+1}, \text { se } \mathrm{z}+1 \geq(1-\beta) \mathrm{s} \mu \\
\mathrm{a}(\mathrm{z}+1)+\mathrm{b}, \text { se } \mathrm{z}+1<(1-\beta) \mathrm{s} \mu
\end{array} ;\right. \\
& \phi_{\mathrm{FBC}}(-\mathrm{z})=\left\{\begin{array}{c}
\frac{1}{-\mathrm{z}}, \text { se }-\mathrm{z} \geq(1-\beta) \mathrm{s} \mu \\
\mathrm{a}(-\mathrm{z})+\mathrm{b}, \text { se }-\mathrm{z}<(1-\beta) \mathrm{s} \mu
\end{array} ;\right.
\end{aligned}
$$

e

$$
\mathrm{H}_{\mathrm{FLBCP}}=\left(\begin{array}{ccccc}
\frac{\partial^{2} \mathrm{FLBCP}}{\partial \mathrm{x}_{1}^{2}} & -4 & 0 & 1 & 2 \mathrm{x}_{1} \\
-4 & \frac{\partial^{2} \mathrm{FLBCP}}{\partial \mathrm{x}_{2}^{2}} & 0 & 1 & -1 \\
0 & 0 & \frac{\partial^{2} \mathrm{FLBCP}}{\partial \mathrm{z}^{2}} & 0 & -1 \\
1 & 1 & 0 & 0 & 0 \\
2 \mathrm{x}_{1} & -1 & -1 & 0 & 0
\end{array}\right)
$$

é a matriz Hessiana com:

$$
\begin{aligned}
& \frac{\partial^{2} \mathrm{FLBCP}}{\partial \mathrm{x}_{1}^{2}}=12\left(\mathrm{x}_{1}-2\right)^{2}+2+2 \lambda^{\mathrm{I}} ; \\
& \frac{\partial^{2} \mathrm{FLBCP}}{\partial \mathrm{x}_{2}^{2}}=8-\mu\left[\phi^{\prime \prime}{ }_{\mathrm{FBC}}\left(\mathrm{x}_{2}-1,5\right)+\phi^{\prime \prime}{ }_{\mathrm{FBC}}\left(2-\mathrm{x}_{2}\right)\right] ; \\
& \frac{\partial^{2} \mathrm{FLBCP}}{\partial \mathrm{z}^{2}}=-\mu\left[\phi^{\prime \prime}{ }_{\mathrm{FBC}}(\mathrm{z}+1)+\phi^{\prime \prime}{ }_{\mathrm{FBC}}(-\mathrm{z})\right] ; \\
& \phi^{\prime \prime}{ }_{\mathrm{FBC}}\left(\mathrm{x}_{2}-1,5\right)=\left\{\begin{array}{c}
-\frac{1}{\left(\mathrm{x}_{2}-1,5\right)^{2}}, \text { se } \mathrm{x}_{2}-1,5 \geq(1-\beta) \mathrm{s} \mu \\
\phi^{\prime \prime}{ }_{\mathrm{FBC}}\left(2-\mathrm{x}_{2}\right)=\left\{\begin{array}{c}
\mathrm{a}, \mathrm{se} \mathrm{x}_{2}-1,5<(1-\beta) \mathrm{s} \mu \\
-\frac{1}{\left(2-\mathrm{x}_{2}\right)^{2}}, \text { se } 2-\mathrm{x}_{2} \geq(1-\beta) \mathrm{s} \mu
\end{array} ;\right.
\end{array}\right.
\end{aligned}
$$




$$
\begin{gathered}
\phi{ }_{\mathrm{FBC}}(\mathrm{z}+1)=\left\{\begin{array}{c}
-\frac{1}{(z+1)^{2}}, \text { se } \mathrm{z}+1 \geq(1-\beta) \mathrm{s} \mu \\
\mathrm{a}, \text { se } \mathrm{z}+1<(1-\beta) \mathrm{s} \mu
\end{array}\right. \\
\phi{ }_{\mathrm{FBC}}(-\mathrm{z})=\left\{\begin{array}{c}
-\frac{1}{(-\mathrm{z})^{2}}, \text { se }-\mathrm{z} \geq(1-\beta) \mathrm{s} \mu \\
\mathrm{a}, \text { se }-\mathrm{z}<(1-\beta) \mathrm{s} \mu
\end{array}\right.
\end{gathered}
$$

\subsubsection{O MÉTODO DA FUNÇÃO LAGRANGIANA BARREIRA MODIFICADA/PENALIDADE}

Para o método da função Lagrangiana barreira modificada/penalidade, associada ao problema (5.1) temos a função Lagrangiana barreira modificada/penalidade:

$$
\begin{aligned}
\text { FLBMP }= & \left(\mathrm{x}_{1}-2\right)^{4}+\left(\mathrm{x}_{1}-2 \mathrm{x}_{2}\right)^{3}-\mu\left[\mathrm{v}^{\mathrm{I}} \phi\left(\mathrm{x}_{2}-1,5\right)+\mathrm{v}^{\mathrm{S}} \phi\left(2-\mathrm{x}_{2}\right)+\xi^{\mathrm{I}} \phi(\mathrm{z}+1)+\right. \\
& \left.+\xi^{\mathrm{S}} \phi(-\mathrm{z})\right]+\lambda^{\mathrm{E}}\left(\mathrm{x}_{1}+\mathrm{x}_{2}-3\right)+\lambda^{\mathrm{I}}\left(\mathrm{x}_{1}{ }^{2}-\mathrm{x}_{2}-\mathrm{z}\right)
\end{aligned}
$$

em que:

$$
\begin{gathered}
\phi\left(x_{2}-1,5\right)=\left\{\begin{array}{c}
\ln \left(\mathrm{s}+\frac{\left(\mathrm{x}_{2}-1,5\right)}{\mu}\right), \text { se } \mathrm{x}_{2}-1,5 \geq-\beta \mathrm{s} \mu \\
\frac{1}{2} \mathrm{q}_{\mathrm{a}}\left(\mathrm{x}_{2}-1,5\right)^{2}+\mathrm{q}_{\mathrm{b}}\left(\mathrm{x}_{2}-1,5\right)+\mathrm{q}_{\mathrm{c}}, \text { se } \mathrm{x}_{2}-1,5<-\beta \mathrm{s} \mu
\end{array} ;\right. \\
\phi\left(2-\mathrm{x}_{2}\right)=\left\{\begin{array}{c}
\ln \left(\mathrm{s}+\frac{\left(2-\mathrm{x}_{2}\right)}{\mu}\right), \text { se } 2-\mathrm{x}_{2} \geq-\beta \mathrm{s} \mu \\
\frac{1}{2} \mathrm{q}_{\mathrm{a}}\left(2-\mathrm{x}_{2}\right)^{2}+\mathrm{q}_{\mathrm{b}}\left(2-\mathrm{x}_{2}\right)+\mathrm{q}_{\mathrm{c}}, \text { se } 2-\mathrm{x}_{2}<-\beta \mathrm{s} \mu
\end{array} ;\right. \\
\phi(\mathrm{z}+1)=\left\{\begin{array}{c}
\ln \left(\mathrm{s}+\frac{(\mathrm{z}+1)}{\mu}\right), \text { se } \mathrm{z}+1 \geq-\beta s \mu \\
\frac{1}{2} \mathrm{q}_{\mathrm{a}}(\mathrm{z}+1)^{2}+\mathrm{q}_{\mathrm{b}}(\mathrm{z}+1)+\mathrm{q}_{\mathrm{c}}, \text { se } \mathrm{z}+1<-\beta \mathrm{s} \mu
\end{array}\right.
\end{gathered}
$$




$$
\begin{aligned}
& \phi(-\mathrm{z})=\left\{\begin{array}{c}
\ln \left(\mathrm{s}+\frac{(-\mathrm{z})}{\mu}\right), \mathrm{se}-\mathrm{z} \geq-\beta \mathrm{s} \mu \\
\frac{1}{2} \mathrm{q}_{\mathrm{a}}(-\mathrm{z})^{2}+\mathrm{q}_{\mathrm{b}}(-\mathrm{z})+\mathrm{q}_{\mathrm{c}}, \mathrm{se}-\mathrm{z}<-\beta \mathrm{s} \mu
\end{array}\right. \\
& \mathrm{q}_{\mathrm{a}}=\frac{-1}{(\mathrm{~s} \mu(1-\beta))^{2}}, \mathrm{q}_{\mathrm{b}}=\frac{1-2 \beta}{\mathrm{s} \mu(1-\beta)^{2}} ; \text { e } \mathrm{q}_{\mathrm{c}}=\frac{\beta(2-3 \beta)}{2(1-\beta)^{2}}+\ln (\mathrm{s}(1-\beta)) .
\end{aligned}
$$

Aplicando as condições de otimalidade à função (5.3), obtemos um sistema de equações não-lineares, cuja solução pode ser determinada pelo método de Newton gerando o seguinte sistema linear:

$$
\mathrm{H}_{\text {FLBMP }} \mathrm{S}=-\mathrm{G}_{\mathrm{FLBMP}}
$$

em que:

$$
\mathrm{G}_{\mathrm{FLBMP}}=\left[\begin{array}{l}
\frac{\partial \mathrm{FLBMP}}{\partial \mathrm{x}_{1}} \\
\frac{\partial \mathrm{FLBMP}}{\partial \mathrm{x}_{2}} \\
\frac{\partial \mathrm{FLBMP}}{\partial \mathrm{z}} \\
\frac{\partial \mathrm{FLBMP}}{\partial \lambda^{\mathrm{E}}} \\
\frac{\partial \mathrm{FLBMP}}{\partial \lambda^{\mathrm{I}}}
\end{array}\right]
$$

é o vetor gradiente com

$$
\begin{aligned}
& \frac{\partial \mathrm{FLBMP}}{\partial \mathrm{x}_{1}}=4\left(\mathrm{x}_{1}-2\right)^{3}+2\left(\mathrm{x}_{1}-2 \mathrm{x}_{2}\right)+\lambda^{\mathrm{E}}+2 \mathrm{x}_{1} \lambda^{\mathrm{I}} ; \\
& \frac{\partial \mathrm{FLBMP}}{\partial \mathrm{x}_{2}}=-4\left(\mathrm{x}_{1}-2 \mathrm{x}_{2}\right)-\mu\left[\mathrm{v}^{\mathrm{I}} \phi^{\prime}\left(\mathrm{x}_{2}-1,5\right)-\mathrm{v}^{\mathrm{S}} \phi^{\prime}\left(2-\mathrm{x}_{2}\right)\right]+\lambda^{\mathrm{E}}-\lambda^{\mathrm{I}} ; \\
& \frac{\partial \mathrm{FLBMP}}{\partial \mathrm{z}}=-\mu\left[\xi^{\mathrm{I}} \phi^{\prime}(\mathrm{z}+1)-\xi^{\mathrm{S}} \phi^{\prime}(-\mathrm{z})\right]-\lambda^{\mathrm{I}} ;
\end{aligned}
$$




$$
\begin{aligned}
& \frac{\partial \text { FLBMP }}{\partial \lambda^{\mathrm{E}}}=\mathrm{x}_{1}+\mathrm{x}_{2}-3 ; \\
& \frac{\partial \mathrm{FLBMP}}{\partial \lambda^{\mathrm{I}}}=\mathrm{x}_{1}^{2}-\mathrm{x}_{2}-\mathrm{z} ; \\
& \phi^{\prime}\left(x_{2}-1,5\right)=\left\{\begin{array}{c}
\frac{1}{\mu s+\left(x_{2}-1,5\right)}, \text { se } x_{2}-1,5 \geq-\beta s \mu \\
q_{a}\left(x_{2}-1,5\right)+q_{b}, \text { se } x_{2}-1,5<-\beta s \mu
\end{array} ;\right. \\
& \phi^{\prime}\left(2-x_{2}\right)=\left\{\begin{array}{c}
\frac{1}{\mu s+\left(2-x_{2}\right)}, \text { se } 2-x_{2} \geq-\beta s \mu \\
q_{a}\left(2-x_{2}\right)+q_{b}, \text { se } 2-x_{2}<-\beta s \mu
\end{array} ;\right. \\
& \phi^{\prime}(z+1)=\left\{\begin{array}{c}
\frac{1}{\mu s+(z+1)}, \text { se } z+1 \geq-\beta s \mu \\
q_{a}(z+1)+q_{b}, \text { se } z+1<-\beta s \mu
\end{array} ;\right. \\
& \phi^{\prime}(-\mathrm{z})=\left\{\begin{array}{c}
\frac{1}{\mu \mathrm{s}+(-\mathrm{z})}, \text { se }-\mathrm{z} z-\beta \mathrm{s} \mu \\
\mathrm{q}_{\mathrm{a}}(-\mathrm{z})+\mathrm{q}_{\mathrm{b}}, \text { se }-\mathrm{z}<-\beta \mathrm{s} \mu
\end{array} ;\right.
\end{aligned}
$$

e

$$
\mathrm{H}_{\mathrm{FLBMP}}=\left(\begin{array}{ccccc}
\frac{\partial^{2} \mathrm{FLBMP}}{\partial \mathrm{x}_{1}^{2}} & -4 & 0 & 1 & 2 \mathrm{x}_{1} \\
-4 & \frac{\partial^{2} \mathrm{FLBMP}}{\partial \mathrm{x}_{2}^{2}} & 0 & 1 & -1 \\
0 & 0 & \frac{\partial^{2} \mathrm{FLBMP}}{\partial \mathrm{z}^{2}} & 0 & -1 \\
1 & 1 & 0 & 0 & 0 \\
2 \mathrm{x}_{1} & -1 & -1 & 0 & 0
\end{array}\right)
$$

é a matriz Hessiana com: 


$$
\begin{aligned}
& \frac{\partial^{2} \text { FLBMP }}{\partial \mathrm{x}_{1}^{2}}=12\left(\mathrm{x}_{1}-2\right)^{2}+2+2 \lambda^{\mathrm{I}} ; \\
& \frac{\partial^{2} \text { FLBMP }}{\partial \mathrm{x}_{2}^{2}}=8-\mu\left[\mathrm{v}^{\mathrm{I}} \phi^{\prime \prime}\left(\mathrm{x}_{2}-1,5\right)+\mathrm{v}^{\mathrm{S}} \phi^{\prime \prime}\left(2-\mathrm{x}_{2}\right)\right] \\
& \frac{\partial^{2} \mathrm{FLBMP}}{\partial \mathrm{z}^{2}}=-\mu\left[\xi^{\mathrm{I}} \phi^{\prime \prime}(\mathrm{z}+1)+\xi^{\mathrm{S}} \phi^{\prime \prime}(-\mathrm{z})\right] \\
& \phi^{\prime \prime}\left(\mathrm{x}_{2}-1,5\right)=\left\{\begin{array}{c}
-\frac{1}{\left[\mu \mathrm{s}+\left(\mathrm{x}_{2}-1,5\right)\right]^{2}}, \text { se } \mathrm{x}_{2}-1,5 \geq-\beta \mathrm{s} \mu \\
\mathrm{q}_{\mathrm{a}}, \text { se } \mathrm{x}_{2}-1,5<-\beta \mathrm{s} \mu
\end{array} ;\right. \\
& \phi^{\prime \prime}\left(2-x_{2}\right)=\left\{\begin{array}{c}
-\frac{1}{\left[\mu s+\left(2-x_{2}\right)\right]^{2}}, \text { se } 2-x_{2} \geq-\beta s \mu \\
q_{a}, \text { se } 2-x_{2}<-\beta s \mu
\end{array} ;\right. \\
& \phi^{\prime \prime}(z+1)=\left\{\begin{array}{c}
-\frac{1}{[\mu s+(z+1)]^{2}}, \text { se } z+1 \geq-\beta s \mu \\
q_{a}, \text { se } z+1<-\beta s \mu
\end{array} ;\right. \\
& \phi^{\prime \prime}(-\mathrm{z})=\left\{\begin{array}{c}
-\frac{1}{[\mu \mathrm{s}+(-\mathrm{z})]^{2}}, \text { se }-\mathrm{z} \geq-\beta \mathrm{s} \mu \\
\mathrm{q}_{\mathrm{a}}, \mathrm{se}-\mathrm{z}<-\beta \mathrm{s} \mu
\end{array} .\right.
\end{aligned}
$$

\section{Testes Numéricos}

Foram realizados 3 testes numéricos, os quais utilizaram o método da FLBMP para diferentes pontos iniciais, implementado em MATLAB ${ }^{\circledR}$. As Tabelas 2, 3, 4, 5, 6, 7, 8, 9, 10, 11, 12 e 13 apresentam o processo de convergência dos testes. 


\section{TESTE 1}

As tabelas 2 e 3 apresentam o processo de inicialização do método FLBMP através do método FLBCP, sendo realizadas duas iterações.

\section{TABELA 2 - Variáveis primais e função objetivo}

\begin{tabular}{c|cccc}
\hline It. & $\mathbf{x}_{\mathbf{1}}$ & $\mathbf{x}_{\mathbf{2}}$ & $\mathbf{z}$ & $\mathbf{F . ~ O b j . ~}$ \\
\hline 0 & 1,00000000 & 2,00000000 & $-1,00000000$ & 10,00000000 \\
1 & 1,31047528 & 1,68952472 & $-0,01318297$ & 4,50504641 \\
2 & 1,31255739 & 1,68744261 & $-0,00194597$ & 4,47652545 \\
\hline
\end{tabular}

TABELA 3 - Variáveis duais e parâmetro de barreira

\begin{tabular}{c|ccc}
\hline It. & $\lambda^{\mathrm{E}}$ & $\lambda^{\mathrm{I}}$ & $\mu$ \\
\hline 0 & 1,00000000 & 1,00000000 & 0,01000000 \\
1 & $-2,02521513$ & 0,91983882 & 0,00100000 \\
2 & $-2,21508741$ & 1,14020966 & - \\
\hline
\end{tabular}

O método FLBCP foi inicializado com: o ponto $\mathrm{x}^{0}=(1 ; 2)$, um ponto factível; a variável de folga $\mathrm{z}=-1$, satisfazendo as restrições de igualdade do problema; os multiplicadores de Lagrange associados às restrições de igualdade, $\lambda^{\mathrm{E}}=1$ e $\lambda^{\mathrm{I}}=1$ respectivamente; com o fator de barreira $\mu=0,01$, sendo $\gamma=10$ seu parâmetro de correção; $\operatorname{com} \beta=0,9$ e $s_{i}=1$. Os parâmetros $\mu, \gamma, \beta$ e $s_{i}$ foram escolhidos de acordo com o capítulo 4. Após as duas iterações, obtivemos um ponto inicial primal-dual e uma estimativa para os multiplicadores de Lagrange associados às restrições de desigualdade, conforme visto no capítulo 4.

Nas tabelas 4 e 5 é apresentado o processo de convergência do método da FLBMP.

TABELA 4 - Variáveis primais, função objetivo e variáveis duais

\begin{tabular}{c|cccccc}
\hline It. & $\mathbf{x}_{\mathbf{1}}$ & $\mathbf{x}_{\mathbf{2}}$ & $\mathbf{z}$ & $\mathbf{F . ~ O b j .}$ & $\lambda^{\mathrm{E}}$ & $\lambda^{\mathbf{I}}$ \\
\hline $\mathbf{0}$ & 1,31255739 & 1,68744261 & $-0,0019000$ & 4,47652545 & $-2,2150874$ & 1,14020966 \\
$\mathbf{1}$ & 1,30517598 & 1,69482402 & 0,00866031 & 4,57810072 & $-4,5019638$ & 3.83576541 \\
$\mathbf{2}$ & 1,30277714 & 1,69722286 & 0,00000543 & 4,61138979 & $-4,5099166$ & 3,85675667 \\
$\mathbf{3}$ & 1,30277564 & 1,69722436 & 0,00000000 & 4,61141071 & $-4,5099222$ & 3,85677011 \\
$\mathbf{4}$ & 1,30277564 & 1,69722436 & 0,00000000 & 4,61141071 & $-4,5099222$ & 3,85677011 \\
\hline
\end{tabular}


TABELA 5 - Estimativas dos multiplicadores de Lagrange e parâmetro de barreira

\begin{tabular}{c|ccccc}
\hline It. & $\mathbf{v}^{\mathbf{I}}$ & $\mathbf{v}^{\mathbf{S}}$ & $\xi^{\mathbf{I}}$ & $\xi^{\mathbf{S}}$ & $\boldsymbol{\mu}$ \\
\hline $\mathbf{0}$ & 0,00533497 & 0,00319941 & 0,00100195 & 0,51388134 & 0,01000000 \\
$\mathbf{1}$ & 0,00026047 & 0,00010151 & 0,00000984 & 3,83583354 & 0,00100000 \\
$\mathbf{2}$ & 0,00000131 & 0,00000033 & 0,00000001 & 3,85676161 & 0,00010000 \\
$\mathbf{3}$ & 0,00000000 & 0,00000000 & 0,00000000 & 3,85680354 & 0,00001000 \\
$\mathbf{4}$ & 0,00000000 & 0,00000000 & 0,00000000 & 3,85722289 & - \\
\hline
\end{tabular}

Houve extrapolação quadrática no ciclo externo do método da FLBCP para a determinação da solução inicial. Foram realizados 12 ciclos internos, dos quais houve extrapolação no $5^{\circ}, 6^{\circ}$ e $7^{\circ}$ ciclos. O método da função Lagrangiana barreira modificada/penalidade foi inicializado com: $x^{0}=(1,31255739 ; 1,68744261)$, um ponto factível; a variável de folga $\mathrm{z}=-0,00194597$; os multiplicadores de Lagrange (variáveis duais) associados às restrições de igualdade, $\lambda^{\mathrm{E}}=-2,21508741 \mathrm{e}$ $\lambda^{\mathrm{I}}=1,14020966$; os multiplicadores de Lagrange associados às restrições de desigualdade: $v^{I}=0,00533497, v^{S}=0.00319941, \quad \xi^{I}=0,00100195 \quad$ e $\xi^{S}=0,51388134$, calculados por (3.56); o fator de barreira $\mu=0,01$, sendo $\gamma=10$ seu parâmetro de correção; com $\beta=0,9$ e $s_{i}=1$. Esses valores encontrados, bem como os fatores e parâmetros utilizados seguem o proposto no capítulo 4. Através dos resultados podemos observar que todo o processo do método da FLBMP necessitou de 4 iterações, mas na $3^{\mathrm{a}}$ iteração as variáveis primais $\left(\mathrm{x}_{1}, \mathrm{x}_{2}\right.$ e $\left.\mathrm{z}\right)$ e as duais $\left(\lambda^{\mathrm{E}}\right.$ e $\left.\lambda^{\mathrm{I}}\right)$ já haviam atingido a sua convergência, porém o processo continuou de modo que os multiplicadores de Lagrange associados às restrições de desigualdade também atinjam a sua convergência. Ocorreu extrapolação quadrática na iteração externa 2. O método realizou 14 ciclos internos, onde ocorreu extrapolação no $1^{\circ}$ ciclo.

\section{TESTE 2}

As tabelas 6 e 7 apresentam o processo de inicialização do método FLBMP através do método FLBCP, sendo realizadas duas iterações.

TABELA 6 - Variáveis primais e função objetivo

\begin{tabular}{c|cccc}
\hline It. & $\mathbf{x}_{\mathbf{1}}$ & $\mathbf{x}_{\mathbf{2}}$ & $\mathbf{Z}$ & $\mathbf{F . ~ O b j . ~}$ \\
\hline $\mathbf{0}$ & 1,10000000 & 1,70000000 & 0,100000000 & 5.94610000 \\
$\mathbf{1}$ & 1,30205850 & 1,69794150 & $-0,00258515$ & 4,62138917 \\
$\mathbf{2}$ & 1,30262181 & 1,69737819 & $-0,00055474$ & 4,61355012 \\
\hline
\end{tabular}


TABELA 7 - Variáveis duais e parâmetro de barreira

\begin{tabular}{c|ccc}
\hline It. & $\lambda^{\mathbf{E}}$ & $\lambda^{\mathbf{I}}$ & $\mu$ \\
\hline $\mathbf{0}$ & $-4,00000000$ & 3,00000000 & 0,01000000 \\
$\mathbf{1}$ & $-4,49966738$ & 3,85821678 & 0,00100000 \\
$\mathbf{2}$ & $-4,49716364$ & 3,83129112 & - \\
\hline
\end{tabular}

O método FLBCP foi inicializado com: $x^{0}=(1,1 ; 1,7)$ e variável de folga $\mathrm{z}=0,1$, valores infactíveis, mas que satisfazem as restrições de desigualdade do problema; os multiplicadores de Lagrange associados às restrições de igualdade (variáveis duais), $\lambda^{\mathrm{E}}=-4$ e $\lambda^{\mathrm{I}}=3$ respectivamente; fator de barreira $\mu=0,01$, sendo $\gamma=10$ seu parâmetro de correção e $\beta=0,9$ e $s_{i}=1$. Após as duas iterações, obtivemos um ponto inicial primal-dual e uma estimativa para os multiplicadores de Lagrange associados às restrições de desigualdade.

Nas tabelas 8 e 9 é apresentado o processo de convergência do método da FLBMP.

TABELA 8 - Variáveis primais, função objetivo e variáveis duais

\begin{tabular}{c|cccccc}
\hline It. & $\mathbf{x}_{\mathbf{1}}$ & $\mathbf{x}_{\mathbf{2}}$ & $\mathbf{z}$ & $\mathbf{F . ~ O b j .}$ & $\lambda^{\mathbf{E}}$ & $\lambda^{\mathbf{I}}$ \\
\hline $\mathbf{0}$ & 1,3026218 & 1,69737819 & $-0,00055474$ & 4,61355012 & $-4,4971636$ & 3,83129112 \\
$\mathbf{1}$ & 1,3042478 & 1,69575215 & 0.00531032 & 4,59096422 & $-4,5050193$ & 3,84386530 \\
$\mathbf{2}$ & 1,3027765 & 1,69722344 & 0.00000334 & 4,61139782 & $-4,5099185$ & 3,85676175 \\
$\mathbf{3}$ & 1,3027756 & 1,69722436 & 0,00000000 & 4,61141071 & $-4,5099222$ & 3,85677011 \\
$\mathbf{4}$ & 1,3027756 & 1,69722436 & 0,00000000 & 4,61141071 & $-4,5099222$ & 3,85677011 \\
$\mathbf{5}$ & 1,3027756 & 1,69722436 & 0,00000000 & 4,61141071 & $-4,5099222$ & 3,85677011 \\
\hline
\end{tabular}

\section{TABELA 9 - Estimativas dos multiplicadores de Lagrange e parâmetro de} barreira

\begin{tabular}{c|ccccc}
\hline It. & $\mathbf{v}^{\mathbf{I}}$ & $\mathbf{v}^{\mathbf{S}}$ & $\xi^{\mathbf{I}}$ & $\xi^{\mathbf{S}}$ & $\boldsymbol{\mu}$ \\
\hline $\mathbf{0}$ & 0,00506642 & 0,00330445 & 0,00100056 & 1,80265600 & 0,01000000 \\
$\mathbf{1}$ & 0,00024624 & 0,00010515 & 0,00000985 & 3,84387596 & 0,00100000 \\
$\mathbf{2}$ & 0,00000124 & 0,00000035 & 0,00000001 & 3,85676684 & 0,00010000 \\
$\mathbf{3}$ & 0,00000000 & 0,00000000 & 0,00000000 & 3,85677492 & 0,00001000 \\
$\mathbf{4}$ & 0,00000000 & 0,00000000 & 0,00000000 & 3,85685577 & 0,00000100 \\
$\mathbf{5}$ & 0,00000000 & 0,00000000 & 0,00000000 & 3,85766441 & - \\
\hline
\end{tabular}

Houve extrapolação quadrática no ciclo externo do método da FLBCP para a determinação da solução inicial. Foram realizados 11 ciclos internos, dos quais houve extrapolação no $6^{\circ}$ ciclo. O método da FLBMP foi inicializado com: $\mathrm{x}^{0}=(1,30262181 ; 1,69737819)$ e variável de folga $\mathrm{z}=-0,00055474$, valores factíveis; multiplicadores de Lagrange associados às restrições de igualdade (variáveis duais), $\lambda^{\mathrm{E}}=-4,49716364$ e $\lambda^{\mathrm{I}}=3,83129112$; multiplicadores de Lagrange associados às 
restrições de desigualdade $\quad \mathrm{v}^{\mathrm{I}}=0,00506642, \quad \mathrm{v}^{\mathrm{S}}=0,00330445, \quad \xi^{\mathrm{I}}=0,00100056 \mathrm{e}$ $\xi^{S}=1,80265600$; fator de barreira $\mu=0,01$, sendo $\gamma=10$ seu parâmetro de correção, $\beta=0,9$ e $s_{i}=1$. Esses valores encontrados, bem como os parâmetros e fatores utilizados, seguem o proposto no capítulo 4 . Através dos resultados podemos observar que todo o processo do método da FLBMP necessitou de 5 iterações, mas na $3^{\mathrm{a}}$ iteração as variáveis primais $\left(x_{1}, x_{2}\right.$ e $\left.z\right)$ e as duais $\left(\lambda^{E}\right.$ e $\left.\lambda^{I}\right)$ já haviam atingido a sua convergência, porém o processo continuou de modo que os multiplicadores de Lagrange associados às restrições de desigualdade também atingissem a sua convergência. Ocorreu extrapolação quadrática na iteração 2. O método realizou 11 ciclos internos, onde ocorreu extrapolação no $1^{\circ}$ ciclo.

\section{TESTE 3}

As tabelas 10 e 11 mostram a inicialização do método FLBMP com o método FLBCP, sendo realizadas duas iterações.

TABELA 10 - Variáveis primais e função objetivo

\begin{tabular}{c|cccc}
\hline It. & $\mathbf{x}_{\mathbf{1}}$ & $\mathbf{x}_{\mathbf{2}}$ & $\mathbf{z}$ & $\mathbf{F . ~ O b j . ~}$ \\
\hline $\mathbf{0}$ & 0,00000000 & 3,00000000 & $-3,00000000$ & 52,00000000 \\
$\mathbf{1}$ & 1,01517301 & 1,98482699 & $-0,95430031$ & 9,66963324 \\
$\mathbf{2}$ & 1,03919440 & 1,96080560 & $-0,88102255$ & 9,16052768 \\
\hline
\end{tabular}

TABELA 11 - Variáveis duais e parâmetro de barreira

\begin{tabular}{c|ccc}
\hline It. & $\lambda^{\mathrm{E}}$ & $\lambda^{\mathrm{I}}$ & $\mu$ \\
\hline $\mathbf{0}$ & 0,00000000 & 0,00000000 & 0,01000000 \\
$\mathbf{1}$ & 0,20047175 & 4,14877014 & 0,00100000 \\
$\mathbf{2}$ & 0,02264064 & 3,99699645 & - \\
\hline
\end{tabular}

O método FLBCP foi inicializado com: $x^{0}=(0 ; 3)$ e variável de folga $z=-3$, valores infactíveis que satisfazem as restrições de igualdade do problema; multiplicadores de Lagrange associados às restrições de igualdade (variáveis duais), $\lambda^{\mathrm{E}}=0$ e $\lambda^{\mathrm{I}}=0$; fator de barreira $\mu=0,01$, sendo $\gamma=10$ seu parâmetro de correção, $\beta=0,9$ e $s_{i}=1$. Após as duas iterações, obtivemos um ponto inicial primal-dual e uma estimativa para os multiplicadores de Lagrange associados às restrições de desigualdade. 
Nas tabelas 12 e 13 é apresentado o processo de convergência do método da FLBMP.

TABELA 12 - Variáveis primais, função objetivo e variáveis duais

\begin{tabular}{c|ccrrrr}
\hline It. & $\mathbf{x}_{\mathbf{1}}$ & \multicolumn{1}{c}{$\mathbf{x}_{\mathbf{2}}$} & \multicolumn{1}{c}{$\mathbf{z}$} & $\mathbf{F}$. Obj. & \multicolumn{1}{c}{$\lambda^{\mathrm{E}}$} & $\lambda^{\mathbf{I}}$ \\
\hline $\mathbf{0}$ & 1,0391944 & 1,96080560 & $-0,88102255$ & 9,16052768 & 0,02264064 & 3,99699645 \\
$\mathbf{1}$ & 1,3824509 & 1,61754907 & 0,29310857 & 3,57774232 & $-4,2129996$ & 3,20018578 \\
$\mathbf{2}$ & 1,3028202 & 1,69717975 & 0,00016084 & 4,61079041 & $-4,5097781$ & 3,85638017 \\
$\mathbf{3}$ & 1,3027756 & 1,69722436 & 0,00000001 & 4,61141067 & $-4,5099222$ & 3,85677009 \\
\hline
\end{tabular}

TABELA 13 - Estimativas dos multiplicadores de Lagrange e parâmetro de barreira

\begin{tabular}{c|ccccc}
\hline It. & $\mathbf{v}^{\mathbf{I}}$ & $\mathbf{v}^{\mathbf{S}}$ & $\xi^{\mathbf{I}}$ & $\xi^{\mathrm{S}}$ & $\mu$ \\
\hline $\mathbf{0}$ & 0,00217011 & 0,02551385 & 0,00840495 & 0,00113504 & 0,01000000 \\
$\mathbf{1}$ & 0,00017014 & 0,00065012 & 0,00006450 & 3,23610985 & 0,00100000 \\
$\mathbf{2}$ & 0,00000086 & 0,00000214 & 0,00000006 & 3,85638057 & 0,00010000 \\
$\mathbf{3}$ & 0,00000000 & 0,00000000 & 0,00000000 & 3,85677544 & - \\
\hline
\end{tabular}

Houve extrapolação quadrática no ciclo externo do método da FLBCP para a determinação da solução inicial. Foram realizados 12 ciclos internos, dos quais houve extrapolação no $1^{\circ}$ ciclo. O método da FLBMP foi inicializado com: $\mathrm{x}^{0}=(1,03919440 ; 1,96080560)$ e variável de folga $\mathrm{z}=-0,88102255$, valores factíveis; multiplicadores de Lagrange associados às restrições de igualdade (variáveis duais) $\lambda^{\mathrm{E}}=0,02264064$ e $\lambda^{\mathrm{I}}=3,99699645$; multiplicadores de Lagrange associados às restrições de desigualdade $v^{I}=0,00217011, v^{S}=0,02551385, \quad \xi^{I}=0,00840495$ e $\xi^{S}=0,00113504$; fator de barreira $\mu=0,01$, sendo $\gamma=10$ seu parâmetro de correção, $\beta=0,9$ e $s_{i}=1$. Esses valores encontrados, bem como os parâmetros e fatores utilizados, seguem o proposto no capítulo 4. Pelos resultados podemos observar que todas as variáveis e parâmetros precisaram de todas as 3 iterações para atingirem a sua convergência. Ocorreu extrapolação quadrática nas iterações 2 e 3 . O método da FLBMP realizou 25 ciclos internos, onde ocorreu extrapolação do $1^{\circ}$ ao $10^{\circ}$ ciclos. 
Nas Figuras 3, 4 e 5 mostramos uma comparação do processo de convergência da FLBMP referente aos Testes 1, 2 e 3. O ponto em preto representa a solução inicial; o em vermelho, a solução ótima e os pontos em verdes, os pontos encontrados nas iterações antes da convergência. As restrições do problema estão na cor magenta e as curvas de níveis da função na cor azul.

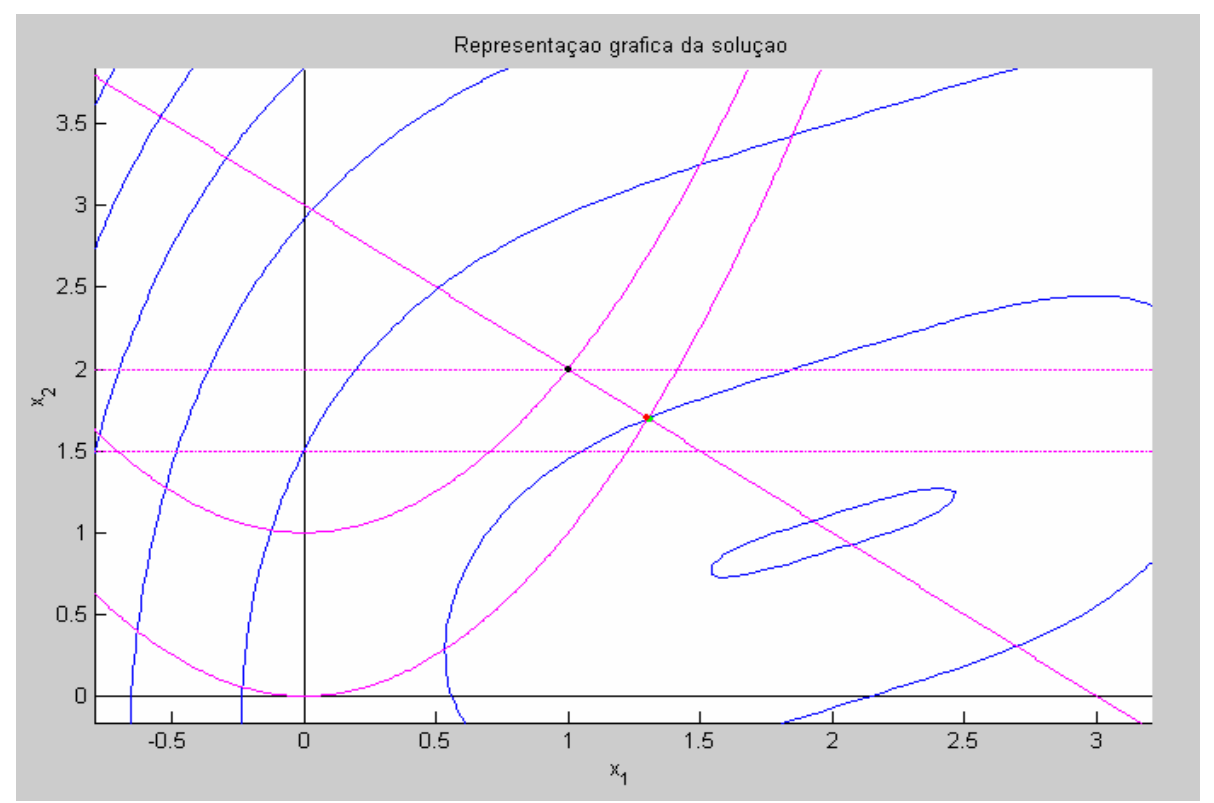

FIGURA 3 - Convergência do método da FLBMP referente ao TESTE 1

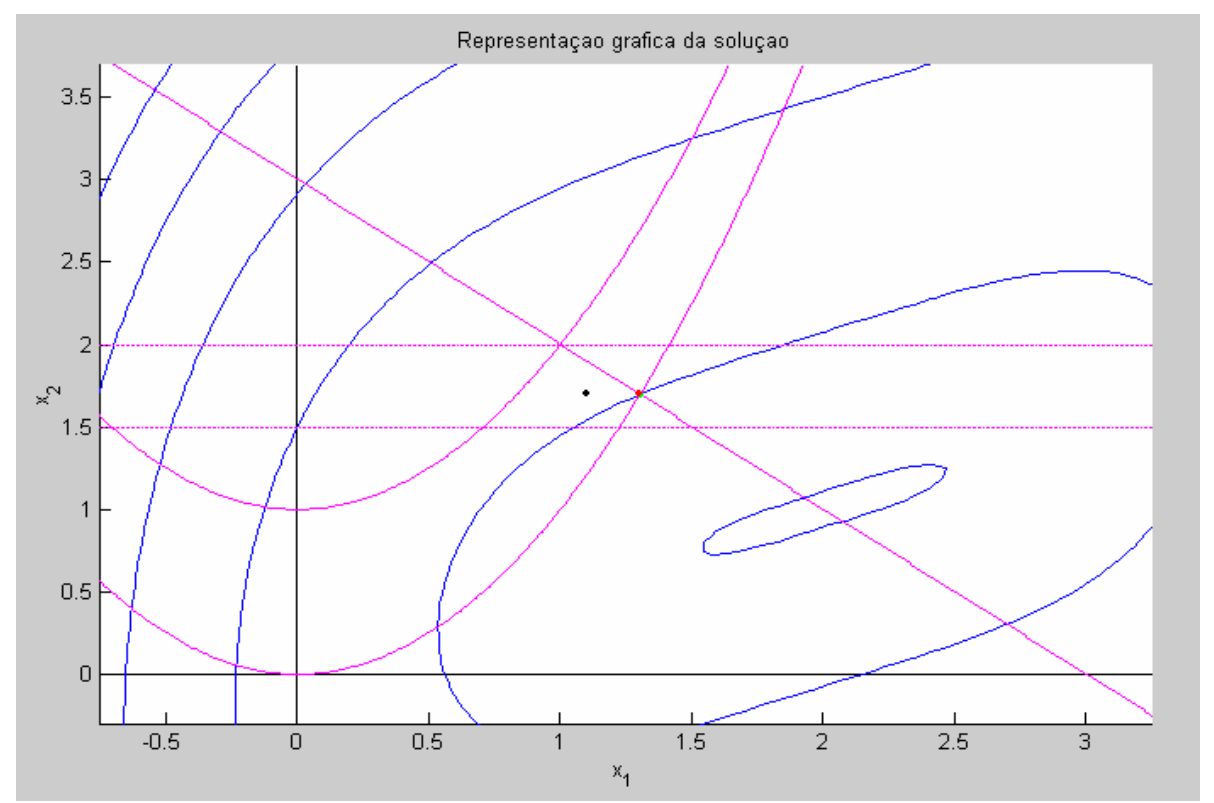

FIGURA 4 - Convergência do método da FLBMP referente ao TESTE 2 


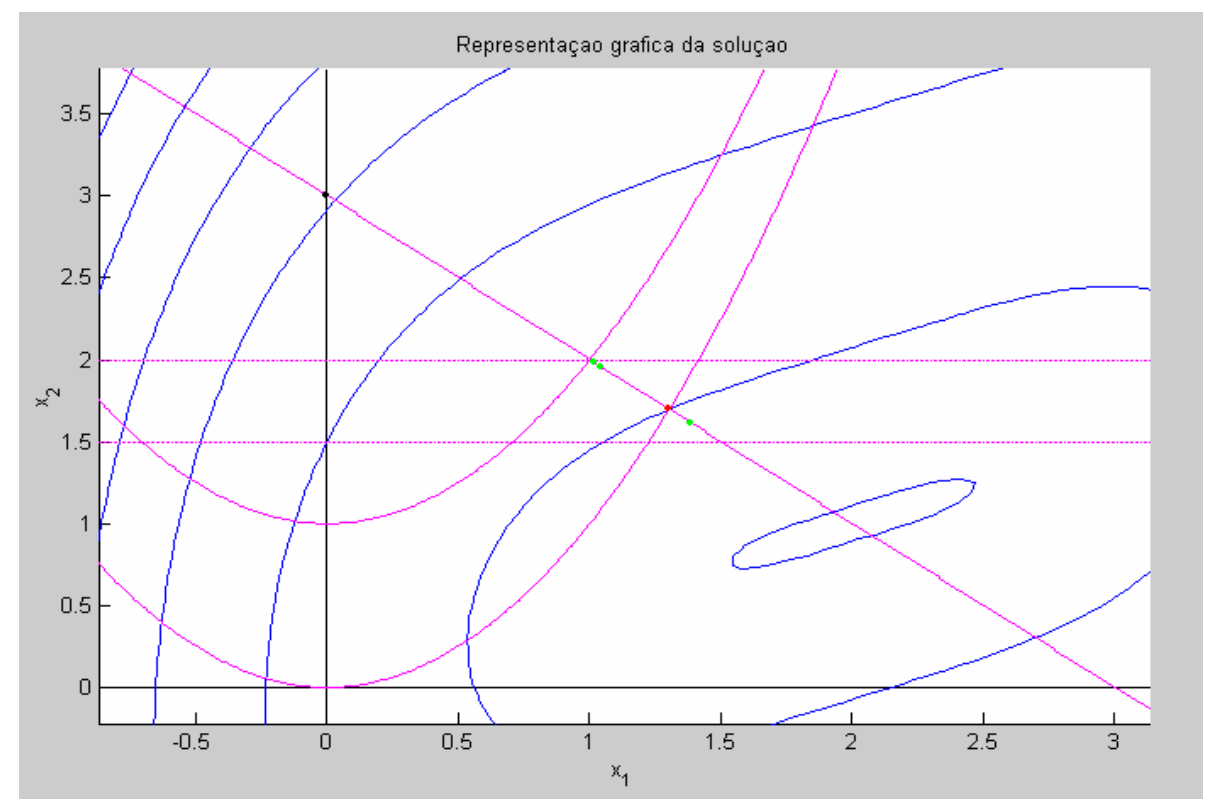

FIGURA 5 - Convergência do método da FLBMP referente ao TESTE 3

Como podemos verificar nas Figuras 3, 4 e 5, dado o ponto inicial, o método proposto procura um ponto satisfazendo as restrições de igualdade, caminhando na região factível. Caso ela saia da região factível ou se inicie com um ponto infactível, o método procura encontrar na próxima iteração um ponto factível, como observamos na figura 3. Podemos observar, também, na figura 2 que se um ponto inicial não satisfaz as restrições de igualdade, o método encontra nas próximas iterações pontos que as satisfaçam, ou seja, as restrições de igualdade sempre serão satisfeitas pelo método. Verificamos, ainda, que a escolha das variáveis primais e duais iniciais, de tal forma que sejam ou não factíveis e satisfaçam ou não as restrições de igualdade, interferem no número de iterações para a convergência ótima. Em todos os casos foi utilizada uma precisão de $\varepsilon=10^{-3}$ e uma tolerância $\eta=10^{-5}$.

\subsection{EXEMPLO 2}

Neste exemplo foi resolvido um grupo de problemas da Engenharia Elétrica conhecidos como problemas de Fluxo de Potência Ótimo (FPO).

O FPO consiste em resolver um conjunto de equações algébricas, não lineares e complexas que resultam da aplicação das leis de Kirchhoff a um sistema com gerações e 
cargas conhecidas. Diferentemente de um problema de fluxo de potência, que necessita da especificação de algumas variáveis tais como: magnitudes de tensão e potência ativa gerada nas barras de geração (barras PV), o FPO trata estas variáveis como passíveis de ajustes. Para tanto, ele geralmente é apresentado como um problema de otimização, onde se procura maximizar ou minimizar um índice de desempenho, atendendo simultaneamente a um conjunto de restrições de igualdade e desigualdade.

Ele é uma ferramenta computacional indicada para determinar o melhor ponto de operação do sistema de potência. Este ponto otimiza um determinado desempenho do sistema e satisfaz todas as restrições físicas e operacionais. O programa computacional de FPO é utilizado por algumas empresas de energia elétrica na área de planejamento e operação do sistema para desenvolvimento de casos base, alocação de reativos, despacho econômico, etc.

O FPO é um termo genérico que envolve uma classe de problemas, em que se destaca o problema de despacho ótimo de potência reativa, o qual é o modelo do problema de FPO utilizado neste trabalho.

Ele pode ser representado matematicamente através de um problema geral de otimização com restrições de igualdade e desigualdade como segue:

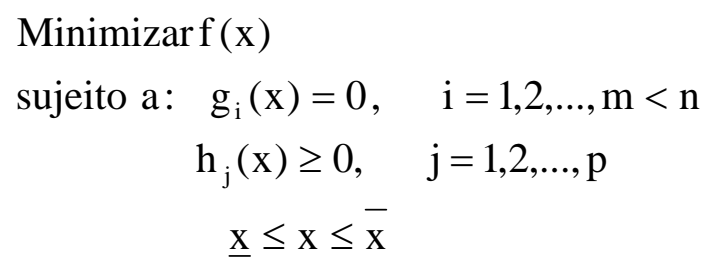

onde :

$\mathrm{x}=(\mathrm{V}, \theta, \mathrm{t}) \in \mathrm{R}^{\mathrm{n}}$ : vetor das variáveis de controle e de estado;

$\mathrm{f}(\mathrm{x})$ : função objetivo que representa o desempenho do sistema (função escalar);

$\mathrm{g}(\mathrm{x}) \in \mathrm{R}^{\mathrm{m}}$ : vetor das equações do fluxo de potência onde $\mathrm{m}<\mathrm{n}$;

$\mathrm{h}(\mathrm{x}) \in \mathrm{R}^{\mathrm{p}}$ : vetor das inequações funcionais do fluxo de potência;

$\underline{\mathrm{h}} \mathrm{e} \overline{\mathrm{h}}$ : limites inferiores e superiores das restrições $\mathrm{h}(\mathrm{x})$, respectivamente;

$\underline{x}$ e $\bar{x}$ : limites inferiores e superiores das variáveis $x$, respectivamente. 
O vetor das variáveis de controle e de estado, $x$, representa a magnitude de tensão (V), o ângulo da tensão $(\theta)$ e o tap dos transformadores (t). A função objetivo, $\mathrm{f}(\mathrm{x})$ pode ser as perdas de potência ativa na transmissão, o custo na geração, entre outras. As restrições de igualdade $\mathrm{g}(\mathrm{x})$ são as equações do fluxo de potência obtidas impondo-se o princípio da conservação de potência em cada barra da rede. As restrições de desigualdade $\mathrm{h}(\mathrm{x})$ representam as restrições funcionais como: a potência reativa nas barras de controle de reativos, potência ativa na barra slack, os fluxos ativos e reativos nas linhas de transmissão, entre outras.

Ao reescrever (5.4) utilizando as equações do fluxo de potência, a função objetivo e as demais restrições, conforme Monticelli (1983), tem-se o seguinte problema de FPO:

$$
\begin{array}{lll}
\operatorname{Minimizar} & \sum_{\mathrm{i}=1}^{\mathrm{NL}} \mathrm{g}_{\mathrm{i}}\left[\mathrm{V}_{\mathrm{k}}^{2}+\mathrm{V}_{\mathrm{m}}^{2}-2 \mathrm{~V}_{\mathrm{k}} \mathrm{V}_{\mathrm{m}} \cos \theta_{\mathrm{km}}\right] & \\
\text { sujeito a } & \mathrm{P}_{\mathrm{k}}^{\mathrm{G}}-\mathrm{P}_{\mathrm{k}}^{\mathrm{C}}-\mathrm{V}_{\mathrm{k}} \sum_{\mathrm{m} \in \mathrm{K}} \mathrm{V}_{\mathrm{m}}\left(\mathrm{G}_{\mathrm{km}} \cos \theta_{\mathrm{km}}+\mathrm{B}_{\mathrm{km}} \operatorname{sen} \theta_{\mathrm{km}}\right)=0, \quad \mathrm{k}=1, \ldots, \text { NBCCR } \\
& \mathrm{Q}_{\mathrm{k}}^{\mathrm{G}}-\mathrm{Q}_{\mathrm{k}}^{\mathrm{C}}-\mathrm{V}_{\mathrm{k}} \sum_{\mathrm{m} \in \mathrm{K}} \mathrm{V}_{\mathrm{m}}\left(\mathrm{G}_{\mathrm{km}} \operatorname{sen} \theta_{\mathrm{km}}+\mathrm{B}_{\mathrm{km}} \cos \theta_{\mathrm{km}}\right)=0, & \mathrm{k}=1, \ldots, \mathrm{NBC} \\
& \underline{\mathrm{Q}}_{\mathrm{k}} \leq \mathrm{V}_{\mathrm{k}} \sum_{\mathrm{m} \in \mathrm{K}} \mathrm{V}_{\mathrm{m}}\left(\mathrm{G}_{\mathrm{km}} \operatorname{sen} \theta_{\mathrm{km}}-\mathrm{B}_{\mathrm{km}} \cos \theta_{\mathrm{km}}\right) \leq \overline{\mathrm{Q}}_{\mathrm{k}}, & \mathrm{k}=1, \ldots, \mathrm{NBCR} \\
& \underline{\mathrm{V}}_{\mathrm{k}} \leq \mathrm{V}_{\mathrm{k}} \leq \overline{\mathrm{V}}_{\mathrm{k}}, & \mathrm{k}=1, \ldots, \mathrm{NB}
\end{array}
$$

em que:

$\mathrm{g}_{\mathrm{i}}$ : condutância da linha i conectada entre a barra k e m;

k e m: barras do sistema;

$\mathrm{V}_{\mathrm{k}}$ e $\mathrm{V}_{\mathrm{m}}$ : magnitudes de tensão nas barras $\mathrm{k}$ e m, respectivamente;

$\theta_{\mathrm{km}}=\theta_{\mathrm{k}}-\theta_{\mathrm{m}}$ : ângulo da tensão na barra $\mathrm{k}$ e m, respectivamente;

$y_{\mathrm{km}}=\mathrm{G}_{\mathrm{km}}+\mathrm{jB}_{\mathrm{km}}$ : admitância da linha entre as barras k e m;

$\mathrm{P}_{\mathrm{k}}{ }^{\mathrm{G}}$ e $\mathrm{P}_{\mathrm{k}}{ }^{\mathrm{C}}$ : potências ativas, gerada e consumida, respectivamente;

$\mathrm{Q}_{\mathrm{k}}{ }^{\mathrm{G}}$ e $\mathrm{Q}_{\mathrm{k}}{ }^{\mathrm{C}}$ : potências reativas, gerada e consumida, respectivamente;

$\mathrm{K}$ - conjunto de todas as barras vizinhas à barra $\mathrm{k}$, incluindo ela mesma.

$\underline{\mathrm{Q}}_{\mathrm{k}}$ e $\overline{\mathrm{Q}}_{\mathrm{k}}$ : limites mínimos e máximos de geração de potência reativa, respectivamente;

$\underline{\mathrm{V}}_{\mathrm{k}}$ e $\overline{\mathrm{V}}_{\mathrm{k}}$ : limites mínimos e máximos da magnitude de tensão, respectivamente;

NL: número de linhas de transmissão;

NB: número de barras do sistema elétrico; 
NBC: número de barras de carga;

NBCR: número de barras de controle de reativo;

NBCCR: número de barras de carga e de controle de reativos.

Para a fase de inicialização dos multiplicadores de Lagrange, seguindo o desenvolvimento matemático apresentado no capítulo 3, obtemos a seguinte função Lagrangiana barreira clássica penalidade associada ao problema (5.5):

$$
\begin{aligned}
\text { FLBCP }=\mathrm{f}(\mathrm{x}) & -\mu\left(\sum_{\mathrm{k}=1}^{\mathrm{NB}} \phi_{\mathrm{FBC}}\left(\mathrm{V}_{\mathrm{k}}-\underline{\mathrm{V}}_{\mathrm{k}}\right)+\sum_{\mathrm{k}=1}^{\mathrm{NB}} \phi_{\mathrm{FBC}}\left(\overline{\mathrm{V}}_{\mathrm{k}}-\mathrm{V}_{\mathrm{k}}\right)\right)+ \\
& -\mu\left(\sum_{\mathrm{n}=1}^{\mathrm{NBCR}} \phi_{\mathrm{FBC}}\left(\mathrm{z}_{\mathrm{n}}-\underline{\mathrm{Q}}_{\mathrm{n}}\right)+\sum_{\mathrm{n}=1}^{\mathrm{NBCR}} \phi_{\mathrm{FBC}}\left(\overline{\mathrm{Q}}_{\mathrm{n}}-\mathrm{z}_{\mathrm{n}}\right)\right)+ \\
& +\sum_{\mathrm{i}=1}^{\mathrm{NB}-1} \lambda^{\mathrm{E}} \mathrm{P}_{\mathrm{i}} \Delta \mathrm{P}_{\mathrm{i}}+\sum_{\mathrm{j}=1}^{\mathrm{NBC}} \lambda^{\mathrm{I}} \mathrm{Q}_{\mathrm{j}} \Delta \mathrm{Q}_{\mathrm{j}}+\sum_{\mathrm{n}=1}^{\mathrm{NBCR}} \lambda^{\mathrm{I}} \mathrm{Q}_{\mathrm{n}}\left(\mathrm{Q}_{\mathrm{n}}-\mathrm{z}_{\mathrm{n}}\right)
\end{aligned}
$$

Associamos também ao problema (5.5), a seguinte função Lagrangiana barreira modificada/ penalidade:

$$
\begin{aligned}
\text { FLBMP }=\mathrm{f}(\mathrm{x}) & -\mu\left(\sum_{\mathrm{k}=1}^{\mathrm{NB}} \mathrm{v}_{\mathrm{k}}^{\mathrm{I}} \phi\left(\mathrm{V}_{\mathrm{k}}-\underline{\mathrm{V}}_{\mathrm{k}}\right)+\sum_{\mathrm{k}=1}^{\mathrm{NB}} \mathrm{v}_{\mathrm{k}}^{\mathrm{S}} \phi\left(\overline{\mathrm{V}}_{\mathrm{k}}-\mathrm{V}_{\mathrm{k}}\right)\right)+ \\
& -\mu\left(\sum_{\mathrm{n}=1}^{\mathrm{NBCR}} \xi_{\mathrm{n}}^{\mathrm{I}} \phi\left(\mathrm{z}_{\mathrm{n}}-\underline{\mathrm{Q}}_{\mathrm{n}}\right)+\sum_{\mathrm{n}=1}^{\mathrm{NBCR}} \xi_{\mathrm{n}}^{\mathrm{S}} \phi\left(\overline{\mathrm{Q}}_{\mathrm{n}}-\mathrm{z}_{\mathrm{n}}\right)\right)+ \\
& +\sum_{\mathrm{i}=1}^{\mathrm{NB}-1} \lambda^{\mathrm{E}} \mathrm{P}_{\mathrm{i}} \Delta \mathrm{P}_{\mathrm{i}}+\sum_{\mathrm{j}=1}^{\mathrm{NBC}} \lambda^{\mathrm{I}} \mathrm{Q}_{\mathrm{j}} \Delta \mathrm{Q}_{\mathrm{j}}+\sum_{\mathrm{n}=1}^{\mathrm{NBCR}} \lambda^{\mathrm{I}} \mathrm{Q}_{\mathrm{n}}\left(\mathrm{Q}_{\mathrm{n}}-\mathrm{z}_{\mathrm{n}}\right)
\end{aligned}
$$

em que:

$$
\begin{aligned}
& \mathrm{f}(\mathrm{x})=\sum_{\mathrm{i}=1}^{\mathrm{NL}} \mathrm{g}_{\mathrm{i}}\left[\mathrm{V}_{\mathrm{k}}^{2}+\mathrm{V}_{\mathrm{m}}^{2}-2 \mathrm{~V}_{\mathrm{k}} \mathrm{V}_{\mathrm{m}} \cos \theta_{\mathrm{km}}\right] ; \\
& \Delta \mathrm{P}_{\mathrm{k}}=\mathrm{P}_{\mathrm{k}}^{\mathrm{G}}-\mathrm{P}_{\mathrm{k}}^{\mathrm{C}}-\mathrm{V}_{\mathrm{k}} \sum_{\mathrm{m} \in \mathrm{K}} \mathrm{V}_{\mathrm{m}}\left(\mathrm{G}_{\mathrm{km}} \cos \theta_{\mathrm{km}}+\mathrm{B}_{\mathrm{km}} \operatorname{sen} \theta_{\mathrm{km}}\right) ; \\
& \Delta \mathrm{Q}_{\mathrm{k}}=\mathrm{Q}_{\mathrm{k}}^{\mathrm{G}}-\mathrm{Q}_{\mathrm{k}}^{\mathrm{C}}-\mathrm{V}_{\mathrm{k}} \sum_{\mathrm{m} \in \mathrm{K}} \mathrm{V}_{\mathrm{m}}\left(\mathrm{G}_{\mathrm{km}} \operatorname{sen} \theta_{\mathrm{km}}+\mathrm{B}_{\mathrm{km}} \cos \theta_{\mathrm{km}}\right) ; \\
& \mathrm{Q}_{\mathrm{k}}=\mathrm{V}_{\mathrm{k}} \sum_{\mathrm{m} \in \mathrm{K}} \mathrm{V}_{\mathrm{m}}\left(\mathrm{G}_{\mathrm{km}} \operatorname{sen} \theta_{\mathrm{km}}-\mathrm{B}_{\mathrm{km}} \cos \theta_{\mathrm{km}}\right) ;
\end{aligned}
$$

$\mu$ : parâmetro de barreira;

Z: variável de folga associada a Q;

$\lambda^{\mathrm{E}} \mathrm{P}_{\mathrm{i}}$ : multiplicador de Lagrange associado à equação $\Delta \mathrm{P}_{\mathrm{i}}=0$; 
$\lambda^{\mathrm{I}} \mathrm{Q}_{\mathrm{j}}$ : multiplicador de Lagrange associado à equação $\Delta \mathrm{Q}_{\mathrm{j}}=0$;

$\lambda^{\mathrm{I}} \mathrm{Q}_{\mathrm{n}}$ : multiplicador de Lagrange associado à equação $\mathrm{Q}_{\mathrm{n}}-\mathrm{z}_{\mathrm{n}}=0$;

$\mathrm{v}_{\mathrm{k}}^{\mathrm{I}}$ : multiplicador de Lagrange associado à inequação $\mathrm{V}_{\mathrm{k}}-\underline{\mathrm{V}}_{\mathrm{k}} \geq 0$;

$\mathrm{v}_{\mathrm{k}}^{\mathrm{S}}$ : multiplicador de Lagrange associado à inequação $\overline{\mathrm{V}}_{\mathrm{k}}-\mathrm{V}_{\mathrm{k}} \geq 0$;

$\xi_{\mathrm{n}}^{\mathrm{I}}$ : multiplicador de Lagrange associado à inequação $\mathrm{z}_{\mathrm{n}}-\underline{\mathrm{Q}}_{\mathrm{n}} \geq 0$;

$\xi_{\mathrm{n}}{ }^{\text {S: }}$ : multiplicador de Lagrange associado à inequação $\overline{\mathrm{Q}}_{\mathrm{n}}-\mathrm{z}_{\mathrm{n}} \geq 0$;

A seguir apresentamos os resultados numéricos obtidos através da aplicação do método da FLBMP em problemas de FPO. Devido a complexidade destes problemas, ficou inviável adotar as condições em que se anulam os gradientes da FLBCP e FLBMP, vistas na Tabela 1, referente aos critérios de convergência do capítulo 4. Os critérios de convergência e parada adotados para substituir tal condição foram a satisfação das restrições de igualdade e desigualdade (factibilidade) e as condições de KKT.

Os sistemas elétricos de potência, utilizados para verificar a eficiência da abordagem proposta foram de 3 barras proposto por Dommel e Tinney (1968); de 14, 30 e 57 barras encontrados em Freris (1968); e de 118 barras, que pode ser encontrado no sitio http://www.ee.washington.edu/research/pstca/. Para cada sistema, a função objetivo a ser minimizada foram as perdas de potência ativa na transmissão, tendo como restrições de igualdade as equações de fluxo de potência e restrições de desigualdade canalizadas das tensões e a restrição funcional de injeção de reativo das barras de controle reativo. As variáveis tap foram, em todos os problemas, consideradas constantes. Em todos os sistemas elétricos utilizados a potência de base foi de 100MVA.

\subsubsection{SISTEMA ELÉTRICO DE 3 BARRAS}

Este exemplo foi proposto por Dommel e Tinney (1968) e tem como finalidade exemplificar a aplicação do método. O sistema de 3 barras tem as seguintes características: 
- 1 barra de referência (Slack - SL);

- 1 barra de controle reativo (CR);

- 1 barra de carga (CG);

- 2 linhas de transmissão.

No modelo do problema de FPO reativo associado ao sistema de 3 barras, tem-se para a barra de controle de reativo, uma equação de balanço da rede $\left(\Delta \mathrm{P}_{2}\right)$. Para a barra de carga tem-se duas equações de balanço da rede $\left(\Delta \mathrm{P}_{3}\right.$ e $\left.\Delta \mathrm{Q}_{3}\right)$. Para a barra de controle de reativo, tem-se a restrição canalizada de geração de potência reativa $\left(\mathrm{Q}_{2}(\mathrm{~V}, \theta)\right)$. Todas as magnitudes de tensão são canalizadas, possuindo limites mínimos e máximos. Essa modelagem é dada por:

Minimizar $\mathrm{f}(\mathrm{V}, \theta)$

sujeitoa: $\Delta \mathrm{P}_{2}=0$

$$
\Delta \mathrm{P}_{3}=0
$$$$
\Delta \mathrm{Q}_{3}=0
$$$$
\underline{\mathrm{V}}_{1} \leq \mathrm{V}_{1} \leq \overline{\mathrm{V}}_{1}
$$$$
\underline{\mathrm{V}}_{2} \leq \mathrm{V}_{2} \leq \overline{\mathrm{V}}_{2}
$$$$
\underline{\mathrm{V}}_{3} \leq \mathrm{V}_{3} \leq \overline{\mathrm{V}}_{3}
$$$$
\underline{\mathrm{Q}}_{2} \leq \mathrm{Q}_{2}(\mathrm{~V}, \theta) \leq \overline{\mathrm{Q}}_{2}
$$

sendo:

$$
\begin{aligned}
& \mathrm{f}(\mathrm{V}, \theta)=\mathrm{g}_{23}\left(\mathrm{~V}_{2}^{2}+\mathrm{V}_{3}^{2}-2 \mathrm{~V}_{2} \mathrm{~V}_{3} \cos \theta_{23}\right)+\mathrm{g}_{31}\left(\mathrm{~V}_{3}^{2}+\mathrm{V}_{1}^{2}-2 \mathrm{~V}_{3} \mathrm{~V}_{1} \cos \theta_{31}\right) \\
& \Delta \mathrm{P}_{2}=\mathrm{P}_{2}^{\mathrm{G}}-\mathrm{P}_{2}^{\mathrm{C}}-\mathrm{V}_{2} \sum_{\mathrm{i}=1}^{3} \mathrm{~V}_{\mathrm{i}}\left(\mathrm{g}_{2 \mathrm{i}} \cos \theta_{2 \mathrm{i}}+\mathrm{b}_{2 \mathrm{i}} \operatorname{sen} \theta_{2 \mathrm{i}}\right) \\
& \Delta \mathrm{P}_{3}=\mathrm{P}_{3}^{\mathrm{G}}-\mathrm{P}_{3}^{\mathrm{C}}-\mathrm{V}_{3} \sum_{\mathrm{i}=1}^{3} \mathrm{~V}_{\mathrm{i}}\left(\mathrm{g}_{3 \mathrm{i}} \cos \theta_{3 \mathrm{i}}+\mathrm{b}_{3 \mathrm{i}} \operatorname{sen} \theta_{3 \mathrm{i}}\right) \\
& \Delta \mathrm{Q}_{3}=\mathrm{Q}_{3}^{\mathrm{G}}-\mathrm{Q}_{3}^{\mathrm{C}}-\mathrm{V}_{3} \sum_{\mathrm{i}=1}^{3} \mathrm{~V}_{\mathrm{i}}\left(\mathrm{g}_{3 \mathrm{i}} \operatorname{sen} \theta_{3 \mathrm{i}}-\mathrm{b}_{3 \mathrm{i}} \cos \theta_{3 \mathrm{i}}\right) \\
& \mathrm{Q}_{2}(\mathrm{~V}, \theta)=\mathrm{V}_{2} \sum_{\mathrm{i}=1}^{3} \mathrm{~V}_{\mathrm{i}}\left(\mathrm{g}_{2 \mathrm{i}} \operatorname{sen} \theta_{2 \mathrm{i}}-\mathrm{b}_{2 \mathrm{i}} \cos \theta_{2 \mathrm{i}}\right)
\end{aligned}
$$


Para a resolução do problema (5.8) pelo método da FLBMP construímos o seguinte problema equivalente, como visto no capítulo 3. Desta forma (5.8) pode ser reescrito da seguinte maneira:

$$
\begin{array}{cl}
\text { Minimizar } & \mathrm{f}(\mathrm{V}, \theta) \\
\text { sujeito a : } & \Delta \mathrm{P}_{2}=0 \\
& \Delta \mathrm{P}_{3}=0 \\
& \Delta \mathrm{Q}_{3}=0 \\
& \mathrm{Q}_{2}-\mathrm{z}_{2}=0 \\
& \mathrm{~V}_{1}-\underline{\mathrm{V}}_{1} \geq 0 \\
\overline{\mathrm{V}}_{1}-\mathrm{V}_{1} \geq 0 \\
\mathrm{~V}_{2}-\underline{\mathrm{V}}_{2} \geq 0 \\
\overline{\mathrm{V}}_{2}-\mathrm{V}_{2} \geq 0 \\
\mathrm{~V}_{3}-\underline{\mathrm{V}}_{3} \geq 0 \\
\overline{\mathrm{V}}_{3}-\mathrm{V}_{3} \geq 0 \\
\mathrm{z}_{2}-\underline{\mathrm{Q}}_{2} \geq 0 \\
\overline{\mathrm{Q}}_{2}-\mathrm{z}_{2} \geq 0
\end{array}
$$

Ao problema (5.9) associamos a FLBCP para determinar as estimativas dos multiplicadores de Lagrange para o método da FLBMP. A FLBCP é dada por:

$$
\begin{aligned}
\text { FLBCP }=\mathrm{f}(\mathrm{V}, \theta)-\mu & \sum_{\mathrm{i}=1}^{3}\left(\phi_{\mathrm{FBC}}\left(\mathrm{V}_{\mathrm{i}}-\underline{\mathrm{V}}_{\mathrm{i}}\right)+\phi_{\mathrm{FBC}}\left(\overline{\mathrm{V}}_{\mathrm{i}}-\mathrm{V}_{\mathrm{i}}\right)\right)-\mu\left(\phi_{\mathrm{FBC}}\left(\mathrm{z}_{2}-\underline{\mathrm{Q}}_{2}\right)+\phi_{\mathrm{FBC}}\left(\overline{\mathrm{Q}}_{2}-\mathrm{z}_{2}\right)\right)+ \\
& +\lambda^{\mathrm{E}} \mathrm{p}_{2}\left(\mathrm{P}_{2}^{\mathrm{G}}-\mathrm{P}_{2}^{\mathrm{C}}-\mathrm{V}_{2} \sum_{\mathrm{i}=1}^{3} \mathrm{~V}_{\mathrm{i}}\left(\mathrm{g}_{2 \mathrm{i}} \cos \theta_{2 \mathrm{i}}+\mathrm{b}_{2 \mathrm{i}} \operatorname{sen} \theta_{2 \mathrm{i}}\right)\right)+ \\
& +\lambda^{\mathrm{E}} \mathrm{p}_{3}\left(\mathrm{P}_{3}^{\mathrm{G}}-\mathrm{P}_{3}^{\mathrm{C}}-\mathrm{V}_{3} \sum_{\mathrm{i}=1}^{3} \mathrm{~V}_{\mathrm{i}}\left(\mathrm{g}_{3 \mathrm{i}} \cos \theta_{3 \mathrm{i}}+\mathrm{b}_{3 \mathrm{i}} \operatorname{sen} \theta_{3 \mathrm{i}}\right)\right)+ \\
& +\lambda^{\mathrm{E}} \mathrm{q}_{3}\left(\mathrm{Q}_{3}^{\mathrm{G}}-\mathrm{Q}_{3}^{\mathrm{C}}-\mathrm{V}_{3} \sum_{\mathrm{i}=1}^{3} \mathrm{~V}_{\mathrm{i}}\left(\mathrm{g}_{3 \mathrm{i}} \operatorname{sen} \theta_{3 \mathrm{i}}-\mathrm{b}_{3 \mathrm{i}} \cos \theta_{3 \mathrm{i}}\right)\right)+ \\
& +\lambda^{\mathrm{I}} \mathrm{q}_{2}\left(\mathrm{~V}_{2} \sum_{\mathrm{i}=1}^{3} \mathrm{~V}_{\mathrm{i}}\left(\mathrm{g}_{2 \mathrm{i}} \operatorname{sen} \theta_{2 \mathrm{i}}-\mathrm{b}_{2 \mathrm{i}} \cos \theta_{2 \mathrm{i}}\right)-\mathrm{z}_{2}\right)
\end{aligned}
$$

em que: 


$$
\begin{aligned}
& \phi_{\mathrm{FBC}}\left(\mathrm{V}_{\mathrm{i}}-\underline{\mathrm{V}}_{\mathrm{i}}\right)=\left\{\begin{array}{c}
\ln \left(\mathrm{V}_{\mathrm{i}}-\underline{\mathrm{V}}_{\mathrm{i}}\right), \text { se } \mathrm{V}_{\mathrm{i}}-\underline{\mathrm{V}}_{\mathrm{i}} \geq(1-\beta) \mathrm{s} \mu \\
\frac{1}{2} \mathrm{a}\left(\mathrm{V}_{\mathrm{i}}-\underline{\mathrm{V}}_{\mathrm{i}}\right)^{2}+\mathrm{b}\left(\mathrm{V}_{\mathrm{i}}-\underline{\mathrm{V}}_{\mathrm{i}}\right)+\mathrm{c}, \text { se } \mathrm{V}_{\mathrm{i}}-\underline{\mathrm{V}}_{\mathrm{i}}<(1-\beta) \mathrm{s} \mu
\end{array} ;\right. \\
& \phi_{\mathrm{FBC}}\left(\overline{\mathrm{V}}_{\mathrm{i}}-\mathrm{V}_{\mathrm{i}}\right)=\left\{\begin{array}{c}
\ln \left(\overline{\mathrm{V}}_{\mathrm{i}}-\mathrm{V}_{\mathrm{i}}\right), \text { se } \overline{\mathrm{V}}_{\mathrm{i}}-\mathrm{V}_{\mathrm{i}} \geq(1-\beta) \mathrm{s} \mu \\
\frac{1}{2} \mathrm{a}\left(\overline{\mathrm{V}}_{\mathrm{i}}-\mathrm{V}_{\mathrm{i}}\right)^{2}+\mathrm{b}\left(\overline{\mathrm{V}}_{\mathrm{i}}-\mathrm{V}_{\mathrm{i}}\right)+\mathrm{c}, \text { se } \overline{\mathrm{V}}_{\mathrm{i}}-\mathrm{V}_{\mathrm{i}}<(1-\beta) \mathrm{s} \mu
\end{array} ;\right. \\
& \phi_{\mathrm{FBC}}\left(\mathrm{z}_{2}-\underline{\mathrm{Q}}_{2}\right)=\left\{\begin{array}{c}
\ln \left(\mathrm{z}_{2}-\underline{\mathrm{Q}}_{2}\right), \text { se } \mathrm{z}_{2}-\underline{\mathrm{Q}}_{2} \geq(1-\beta) \mathrm{s} \mu \\
\frac{1}{2} \mathrm{a}\left(\mathrm{z}_{2}-\underline{\mathrm{Q}}_{2}\right)^{2}+\mathrm{b}\left(\mathrm{z}_{2}-\underline{\mathrm{Q}}_{2}\right)+\mathrm{c}, \text { se } \mathrm{z}_{2}-\underline{\mathrm{Q}}_{2}<(1-\beta) \mathrm{s} \mu
\end{array} ;\right. \\
& \phi_{\mathrm{FBC}}\left(\overline{\mathrm{Q}}_{2}-\mathrm{z}_{2}\right)=\left\{\begin{array}{c}
\ln \left(\overline{\mathrm{Q}}_{2}-\mathrm{z}_{2}\right), \text { se } \overline{\mathrm{Q}}_{2}-\mathrm{z}_{2} \geq(1-\beta) \mathrm{s} \mu \\
\frac{1}{2} \mathrm{a}\left(\overline{\mathrm{Q}}_{2}-\mathrm{z}_{2}\right)^{2}+\mathrm{b}\left(\overline{\mathrm{Q}}_{2}-\mathrm{z}_{2}\right)+\mathrm{c} \mathrm{se} \overline{\mathrm{Q}}_{2}-\mathrm{z}_{2}<(1-\beta) \mathrm{s} \mu
\end{array} ;\right. \\
& \mathrm{a}=-\frac{1}{((1-\beta) \mathrm{s} \mu)^{2}}, \mathrm{~b}=\frac{2}{(1-\beta) \mathrm{s} \mu} \text { e c }=\ln ((1-\beta) s \mu)-\frac{3}{2} .
\end{aligned}
$$

Após a inicialização com o método da FLBCP, para determinar uma solução inicial para o problema, associamos ao problema (5.9) a FLBMP:

$$
\begin{aligned}
\operatorname{FLBMP}=\mathrm{f}(\mathrm{V}, \theta)-\mu & \sum_{\mathrm{i}=1}^{3}\left(\mathrm{v}_{\mathrm{i}}^{\mathrm{I}} \phi\left(\mathrm{V}_{\mathrm{i}}-\underline{\mathrm{V}}_{\mathrm{i}}\right)+\mathrm{v}_{\mathrm{i}}^{\mathrm{S}} \phi\left(\overline{\mathrm{V}}_{\mathrm{i}}-\mathrm{V}_{\mathrm{i}}\right)\right)-\mu\left(\xi_{\mathrm{i}}^{\mathrm{I}} \phi\left(\mathrm{z}_{2}-\underline{\mathrm{Q}}_{2}\right)+\xi_{\mathrm{i}}^{\mathrm{S}} \phi\left(\overline{\mathrm{Q}}_{2}-\mathrm{z}_{2}\right)\right)+ \\
& +\lambda^{\mathrm{E}} \mathrm{p}_{2}\left(\mathrm{P}_{2}^{\mathrm{G}}-\mathrm{P}_{2}^{\mathrm{C}}-\mathrm{V}_{2} \sum_{\mathrm{i}=1}^{3} \mathrm{~V}_{\mathrm{i}}\left(\mathrm{g}_{2 \mathrm{i}} \cos \theta_{2 \mathrm{i}}+\mathrm{b}_{2 \mathrm{i}} \operatorname{sen} \theta_{2 \mathrm{i}}\right)\right)+ \\
& +\lambda^{\mathrm{E}} \mathrm{p}_{3}\left(\mathrm{P}_{3}^{\mathrm{G}}-\mathrm{P}_{3}^{\mathrm{C}}-\mathrm{V}_{3} \sum_{\mathrm{i}=1}^{3} \mathrm{~V}_{\mathrm{i}}\left(\mathrm{g}_{3 \mathrm{i}} \cos \theta_{3 \mathrm{i}}+\mathrm{b}_{3 \mathrm{i}} \operatorname{sen} \theta_{3 \mathrm{i}}\right)\right)+ \\
& +\lambda^{\mathrm{E}} \mathrm{q}_{3}\left(\mathrm{Q}_{3}^{\mathrm{G}}-\mathrm{Q}_{3}^{\mathrm{C}}-\mathrm{V}_{3} \sum_{\mathrm{i}=1}^{3} \mathrm{~V}_{\mathrm{i}}\left(\mathrm{g}_{3 \mathrm{i}} \operatorname{sen} \theta_{3 \mathrm{i}}-\mathrm{b}_{3 \mathrm{i}} \cos \theta_{3 \mathrm{i}}\right)\right)+ \\
& +\lambda^{\mathrm{I}} \mathrm{q}_{2}\left(\mathrm{~V}_{2} \sum_{\mathrm{i}=1}^{3} \mathrm{~V}_{\mathrm{i}}\left(\mathrm{g}_{2 \mathrm{i}} \operatorname{sen} \theta_{2 \mathrm{i}}-\mathrm{b}_{2 \mathrm{i}} \cos \theta_{2 \mathrm{i}}\right)-\mathrm{z}_{2}\right)
\end{aligned}
$$

em que: 


$$
\begin{aligned}
& \phi\left(V_{i}-\underline{V}_{i}\right)=\left\{\begin{array}{c}
\ln \left(s+\frac{V_{i}-\underline{V}_{i}}{\mu}\right), \text { se } V_{i}-\underline{V}_{i} \geq-\beta s \mu \\
\frac{1}{2} q_{a}\left(V_{i}-\underline{V}_{i}\right)^{2}+q_{b}\left(V_{i}-\underline{V}_{i}\right)+q_{c}, \text { se } V_{i}-\underline{V}_{i}<-\beta s \mu
\end{array} ;\right. \\
& \phi\left(\overline{\mathrm{V}}_{\mathrm{i}}-\mathrm{V}_{\mathrm{i}}\right)=\left\{\begin{array}{c}
\ln \left(\mathrm{s}+\frac{\overline{\mathrm{V}}_{\mathrm{i}}-\mathrm{V}_{\mathrm{i}}}{\mu}\right), \text { se } \overline{\mathrm{V}}_{\mathrm{i}}-\mathrm{V}_{\mathrm{i}} \geq-\beta \mathrm{s} \mu \\
\frac{1}{2} \mathrm{q}_{\mathrm{a}}\left(\overline{\mathrm{V}}_{\mathrm{i}}-\mathrm{V}_{\mathrm{i}}\right)^{2}+\mathrm{q}_{\mathrm{b}}\left(\overline{\mathrm{V}}_{\mathrm{i}}-\mathrm{V}_{\mathrm{i}}\right)+\mathrm{q}_{\mathrm{c}}, \text { se } \overline{\mathrm{V}}_{\mathrm{i}}-\mathrm{V}_{\mathrm{i}}<-\beta s \mu
\end{array} ;\right. \\
& \phi\left(\mathrm{z}_{2}-\underline{\mathrm{Q}}_{2}\right)=\left\{\begin{array}{c}
\ln \left(\mathrm{s}+\frac{\mathrm{z}_{2}-\underline{\mathrm{Q}}_{2}}{\mu}\right), \text { se } \mathrm{z}_{2}-\underline{\mathrm{Q}}_{2} \geq-\beta \mathrm{s} \mu \\
\frac{1}{2} \mathrm{q}_{\mathrm{a}}\left(\mathrm{z}_{2}-\underline{\mathrm{Q}}_{2}\right)^{2}+\mathrm{q}_{\mathrm{b}}\left(\mathrm{z}_{2}-\underline{\mathrm{Q}}_{2}\right)+\mathrm{q}_{\mathrm{c}}, \text { se } \mathrm{z}_{2}-\underline{\mathrm{Q}}_{2}<-\beta s \mu
\end{array} ;\right. \\
& \phi\left(\overline{\mathrm{Q}}_{2}-\mathrm{z}_{2}\right)=\left\{\begin{array}{c}
\ln \left(\mathrm{s}+\frac{\overline{\mathrm{Q}}_{2}-\mathrm{z}_{2}}{\mu}\right), \text { se } \overline{\mathrm{Q}}_{2}-\mathrm{z}_{2} \geq-\beta \mathrm{s} \mu \\
\frac{1}{2} \mathrm{q}_{\mathrm{a}}\left(\overline{\mathrm{Q}}_{2}-\mathrm{z}_{2}\right)^{2}+\mathrm{q}_{\mathrm{b}}\left(\overline{\mathrm{Q}}_{2}-\mathrm{z}_{2}\right)+\mathrm{q}_{\mathrm{c}} \text { se } \overline{\mathrm{Q}}_{2}-\mathrm{z}_{2}<-\beta \mathrm{s} \mu
\end{array} ;\right. \\
& \mathrm{q}_{\mathrm{a}}=\frac{-1}{(\mathrm{~s} \mu(1-\beta))^{2}}, \mathrm{q}_{\mathrm{b}}=\frac{1-2 \beta}{\mathrm{s} \mu(1-\beta)^{2}} \text { e } \mathrm{q}_{\mathrm{c}}=\frac{\beta(2-3 \beta)}{2(1-\beta)^{2}}+\ln (\mathrm{s}(1-\beta))
\end{aligned}
$$

Apresentamos passo a passo o processo de convergência para o sistema de 3 barras, destacando o valor de todas as variáveis do problema, do parâmetro de barreira, dos multiplicadores de Lagrange e dos valores das potências ativas e reativas nas barras. Os limites da tensão na barra 3 foram escolhidos muito próximos, para que a tensão final fosse praticamente de 1.0 p.u., como proposto por Dommel e Tinney (1968). Consideramos $\gamma=2$ para a atualização do parâmetro de barreira, e este foi atualizado através de (3.41).

\section{Estado inicial do sistema}

O estado inicial do sistema e os limites utilizados para o nível de tensão e injeção de reativo são mostrados nas Tabelas 14 e 15. Os valores iniciais das variáveis 
de folga e dos multiplicadores de Lagrange para as restrições de igualdade são mostrados na tabela 16.

TABELA 14 - Estado inicial do sistema de 3 barras

\begin{tabular}{c|ccccccc}
\hline Barra & Tipo & V(p.u.) & Ang(rad) & $\mathbf{P}^{\mathrm{G}}$ (p.u.) & $\mathbf{P}^{\mathrm{C}}$ (p.u.) & $\mathbf{Q}^{\mathrm{G}}$ (p.u.) & $\mathbf{Q}^{\mathrm{C}}$ (p.u.) \\
\hline $\mathbf{1}$ & SL & 1,0000 & 0,0000 & - & - & - & - \\
$\mathbf{2}$ & CR & 1,0000 & 0,0000 & 1,7000 & 0,0000 & - & - \\
$\mathbf{3}$ & CG & 1,0000 & 0,0000 & 0,0000 & 2,0000 & 0,0000 & 1,0000 \\
\hline
\end{tabular}

TABELA 15 - Limites para as tensões e reativos

\begin{tabular}{c|cccc}
\hline Barra & $\underline{\mathbf{V}}$ (p.u.) & $\overline{\mathbf{V}}$ (p.u.) & $\underline{\mathbf{Q} \text { (p.u.) }}$ & $\overline{\mathbf{Q}}$ (p.u.) \\
\hline $\mathbf{1}$ & 0,9500 & 1,1000 & $-99,9900$ & 99,9900 \\
$\mathbf{2}$ & 0,9500 & 1,2000 & $-99,9900$ & 99,9900 \\
$\mathbf{3}$ & 0,9900 & 1,0100 & - & - \\
\hline
\end{tabular}

TABELA 16 - Valores iniciais das variáveis de folga e dos multiplicadores de Lagrange

\begin{tabular}{c|cccc}
\hline Barra & $\mathbf{z}$ & $\lambda^{\mathrm{E}} \mathbf{p}$ & $\lambda^{\mathrm{E}} \mathbf{q}$ & $\lambda^{\mathrm{I}} \mathbf{q}$ \\
\hline $\mathbf{1}$ & - & - & - & - \\
$\mathbf{2}$ & 0,0000 & 1,0000 & - & 1,0000 \\
$\mathbf{3}$ & - & 1,0000 & 1,0000 & - \\
\hline
\end{tabular}

Perdas ativas iniciais do sistema: 4,1406 MW.

Estes valores foram utilizados na fase de inicialização com o método da FLBCP a fim de encontrar estimativas para os multiplicadores de Lagrange das restrições de desigualdade utilizados no método da FLBMP. Nesta fase, foram realizadas duas iterações.

\section{Método da FLBCP}

Iteração 1

O valor na iteração 1 do parâmetro de barreira é $\mu=0,01$. 
TABELA 17 - Variáveis do sistema de 3 barras na iteração 1

\begin{tabular}{c|cccccc}
\hline Barra & Tipo & V(p.u.) & Ang(rad) & $\Delta$ P(p.u.).10 & $\Delta$ Q(p.u.).10 & Q(V,0) (p.u.) \\
\hline $\mathbf{1}$ & SL & 1,0755 & 0,0000 & - & - & - \\
$\mathbf{2}$ & CR & 1,1232 & 0,0807 & 0,0038 & - & 0,9769 \\
$\mathbf{3}$ & CG & 1,0010 & $-0,0199$ & $-0,6003$ & 1,5931 & - \\
\hline
\end{tabular}

TABELA 18 - Variáveis de folga e multiplicadores de Lagrange

\begin{tabular}{c|cccc}
\hline Barra & $\mathbf{z}$ & $\lambda^{\mathbf{E}} \mathbf{p}$ & $\lambda^{\mathbf{E}} \mathbf{q}$ & $\lambda^{\mathbf{I}} \mathbf{q}$ \\
\hline $\mathbf{1}$ & - & - & - & - \\
$\mathbf{2}$ & 0,9764 & 0,0326 & - & 0,0000 \\
$\mathbf{3}$ & - & $-0,0767$ & $-0,0534$ & - \\
\hline
\end{tabular}

Perdas ativas do sistema: 12,8973 MW.

Iteração 2

O valor na iteração 2 do parâmetro de barreira é $\mu=0,001$.

TABELA 19 - Variáveis do sistema de 3 barras na iteração 2

\begin{tabular}{c|cccccc}
\hline Barra & Tipo & V(p.u.) & Ang(rad) & $\Delta$ P(p.u.).10 & $\Delta$ Q(p.u.).10 & Q(V,0) (p.u.) \\
\hline $\mathbf{1}$ & SL & 1,0756 & 0,0000 & - & - & - \\
$\mathbf{2}$ & CR & 1,1311 & 0,0742 & 0,1061 & - & 1,0209 \\
$\mathbf{3}$ & CG & 1,0063 & $-0,0237$ & $-0,1322$ & $-5,2632$ & - \\
\hline
\end{tabular}

TABELA 20 - Variáveis de folga e multiplicadores de Lagrange

\begin{tabular}{c|cccc}
\hline Barra & $\mathbf{z}$ & $\lambda^{\mathbf{E}} \mathbf{p}$ & $\lambda^{\mathbf{E}} \mathbf{q}$ & $\lambda^{\mathbf{I}} \mathbf{q}$ \\
\hline $\mathbf{1}$ & - & - & - & - \\
$\mathbf{2}$ & 1,0209 & 0,0247 & - & 0,0000 \\
$\mathbf{3}$ & - & $-0,0815$ & $-0,0509$ & - \\
\hline
\end{tabular}

Perdas ativas do sistema: 12,7593 MW.

Esses valores foram utilizados para calcular as estimativas dos multiplicadores de Lagrange conforme (4.56) para as restrições de desigualdade (v $\mathrm{v}^{\mathrm{I}}, \mathrm{v}^{\mathrm{S}}, \xi^{\mathrm{I}}$ e $\left.\xi^{\mathrm{S}}\right)$ bem como se tornaram os valores iniciais no método da FLBMP.

\section{Método da FLBMP}

Iteração 1 
O valor na iteração 1 do parâmetro de barreira é $\mu=0,01$.

TABELA 21 - Variáveis do sistema de 3 barras na iteração 1

\begin{tabular}{c|cccccc}
\hline Barra & Tipo & V(p.u.) & Ang(rad) & $\Delta$ P(p.u.).10 & $\Delta$ Q(p.u.).10 & Q(V,, ) (p.u.) \\
\hline $\mathbf{1}$ & SL & 1,0883 & 0,0000 & - & - & - \\
$\mathbf{2}$ & CR & 1,1421 & 0,0739 & 0,4389 & - & 1,0074 \\
$\mathbf{3}$ & CG & 1,0194 & $-0,0223$ & $-0,6992$ & $-4,0378$ & - \\
\hline
\end{tabular}

TABELA 22 - Variáveis de folga e multiplicadores de Lagrange

\begin{tabular}{c|cccc}
\hline Barra & $\mathbf{z}$ & $\lambda^{\mathrm{E}} \mathbf{p}$ & $\lambda^{\mathrm{E}} \mathbf{q}$ & $\lambda^{\mathrm{I}} \mathbf{q}$ \\
\hline $\mathbf{1}$ & - & - & - & - \\
$\mathbf{2}$ & 1,0074 & 0,0271 & - & 0,0000 \\
$\mathbf{3}$ & - & $-0,0736$ & $-0,0468$ & - \\
\hline
\end{tabular}

TABELA 23 - Estimativas dos multiplicadores de Lagrange

\begin{tabular}{c|cccc}
\hline Barra & $\mathbf{v}^{\mathbf{I}}$ & $\mathbf{v}^{\mathbf{S}}$ & $\xi^{\mathbf{I}}$ & $\xi^{\mathbf{S}}$ \\
\hline $\mathbf{1}$ & 0,0001 & 0,0019 & - & - \\
$\mathbf{2}$ & 0,0000 & 0,0002 & 0,0000 & 0,0000 \\
$\mathbf{3}$ & 0,0016 & 0,3613 & - & - \\
\hline
\end{tabular}

Perdas ativas do sistema: 12,4383 MW.

Iteração 2

O valor na iteração 2 do parâmetro de barreira é $\mu=0,001$.

TABELA 24 - Variáveis do sistema de 3 barras na iteração 2

\begin{tabular}{c|cccccc}
\hline Barra & Tipo & V(p.u.) & Ang(rad) & $\Delta$ P(p.u.).10 & $\Delta$ Q(p.u.).10 & Q(V, $\theta)$ (p.u.) \\
\hline $\mathbf{1}$ & SL & 1,0799 & 0,0000 & - & - & - \\
$\mathbf{2}$ & CR & 1,1331 & 0,0755 & 0,3775 & - & 1,0063 \\
$\mathbf{3}$ & CG & 1,0096 & $-0,0224$ & $-0,4879$ & $-2,6230$ & - \\
\hline
\end{tabular}

TABELA 25 - Variáveis de folga e multiplicadores de Lagrange

\begin{tabular}{c|cccc}
\hline Barra & $\mathbf{z}$ & $\lambda^{\mathrm{E}} \mathbf{p}$ & $\lambda^{\mathrm{E}} \mathbf{q}$ & $\lambda^{\mathrm{I}} \mathbf{q}$ \\
\hline $\mathbf{1}$ & - & - & - & - \\
$\mathbf{2}$ & 1,0063 & 0,0275 & - & 0,0000 \\
$\mathbf{3}$ & - & $-0,0775$ & $-0,0490$ & - \\
\hline
\end{tabular}


TABELA 26 - Estimativas dos multiplicadores de Lagrange

\begin{tabular}{c|cccc}
\hline Barra & $\mathbf{v}^{\mathbf{I}}$ & $\mathbf{v}^{\mathbf{S}}$ & $\xi^{\mathbf{I}}$ & $\xi^{\mathbf{S}}$ \\
\hline $\mathbf{1}$ & 0,0000 & 0,0001 & - & - \\
$\mathbf{2}$ & 0,0000 & 0,0000 & 0,0000 & 0,0000 \\
$\mathbf{3}$ & 0,0001 & 0,2806 & - & - \\
\hline
\end{tabular}

Perdas ativas do sistema: 12,6765 MW.

Iteração 3

O valor na iteração 3 do parâmetro de barreira é $\mu=0,0001$.

TABELA 27 - Variáveis do sistema de 3 barras na iteração 3

\begin{tabular}{c|cccccc}
\hline Barra & Tipo & V(p.u.) & Ang(rad) & $\Delta$ P(p.u.).10 & $\Delta$ Q(p.u.).10 & Q(V, $\mathbf{1 0}^{-1}$ (p.u.) \\
\hline $\mathbf{1}$ & SL & 1,0802 & 0,0000 & - & - & - \\
$\mathbf{2}$ & CR & 1,1334 & 0,0755 & 0,1205 & - & 1,0061 \\
$\mathbf{3}$ & CG & 1,0100 & $-0,0224$ & $-0,1558$ & $-8,3595$ & - \\
\hline
\end{tabular}

TABELA 28 - Variáveis de folga e multiplicadores de Lagrange

\begin{tabular}{c|cccc}
\hline Barra & $\mathbf{z}$ & $\lambda^{\mathbf{E}} \mathbf{p}$ & $\lambda^{\mathbf{E}} \mathbf{q}$ & $\lambda^{\mathbf{I}} \mathbf{q}$ \\
\hline $\mathbf{1}$ & - & - & - & - \\
$\mathbf{2}$ & 1,0061 & 0,0275 & - & 0,0000 \\
$\mathbf{3}$ & - & $-0,0774$ & $-0,0489$ & - \\
\hline
\end{tabular}

TABELA 29 - Estimativas dos multiplicadores de Lagrange

\begin{tabular}{c|cccc}
\hline Barra & $\mathbf{v}^{\mathbf{I}}$ & $\mathbf{v}^{\mathbf{S}}$ & $\xi^{\mathbf{I}}$ & $\xi^{\mathbf{S}}$ \\
\hline $\mathbf{1}$ & 0,0000 & 0,0000 & - & - \\
$\mathbf{2}$ & 0,0000 & 0,0000 & 0,0000 & 0,0000 \\
$\mathbf{3}$ & 0,0000 & 0,2460 & - & - \\
\hline
\end{tabular}

Perdas ativas do sistema: 12,6672 MW.

Iteração 4

O valor na iteração 4 do parâmetro de barreira é $\mu=0,00001$. 
TABELA 30 - Variáveis do sistema de 3 barras na iteração 4

\begin{tabular}{c|cccccc}
\hline Barra & Tipo & V(p.u.) & Ang(rad) & $\Delta$ P(p.u.).10 & $\Delta$ Q(p.u.).10 & Q(V, (1) $^{-11}$ (p.u.) \\
\hline $\mathbf{1}$ & SL & 1,0803 & 0,0000 & - & - & - \\
$\mathbf{2}$ & CR & 1,1334 & 0,0755 & 0,1432 & - & 1,0061 \\
$\mathbf{3}$ & CG & 1,0100 & $-0,0224$ & $-0,1849$ & $-9,7700$ & - \\
\hline
\end{tabular}

TABELA 31 - Variáveis de folga e multiplicadores de Lagrange

\begin{tabular}{c|cccc}
\hline Barra & $\mathbf{z}$ & $\lambda^{\mathrm{E}} \mathbf{p}$ & $\lambda^{\mathrm{E}} \mathbf{q}$ & $\lambda^{\mathrm{I}} \mathbf{q}$ \\
\hline $\mathbf{1}$ & - & - & - & - \\
$\mathbf{2}$ & 1,0061 & 0,0275 & - & 0,0000 \\
$\mathbf{3}$ & - & $-0,0774$ & $-0,0489$ & - \\
\hline
\end{tabular}

TABELA 32 - Estimativas dos multiplicadores de Lagrange

\begin{tabular}{c|cccc}
\hline Barra & $\mathbf{v}^{\mathbf{I}}$ & $\mathbf{v}^{\mathbf{S}}$ & $\xi^{\mathbf{I}}$ & $\xi^{\mathbf{S}}$ \\
\hline $\mathbf{1}$ & 0,0000 & 0,0000 & - & - \\
$\mathbf{2}$ & 0,0000 & 0,0000 & 0,0000 & 0,0000 \\
$\mathbf{3}$ & 0,0000 & 0,2466 & - & - \\
\hline
\end{tabular}

Perdas ativas do sistema: 12,6668 MW.

O sistema convergiu em 4 iterações, com uma precisão de $10^{-5}$ p.u. Na solução do problema, as restrições de igualdade e desigualdade e as condições de KKT estão satisfeitas.

As tabelas 33 e 34 mostram o processo de convergência da função objetivo para o método da FLBCP e para o método da FLBMP. Os dados apresentados nestas tabelas não estão em p.u.

TABELA 33 - Convergência da Função Objetivo no método da FLBCP

\begin{tabular}{c|ccc}
\hline Iteração & Função Objetivo (MW) & $\mathbf{M A X}(\Delta \mathbf{P})$ & $\mathbf{M A X}(\Delta \mathbf{Q})$ \\
\hline $\mathbf{0}$ & 4,140646 & 117,115145 & 159,070224 \\
$\mathbf{1}$ & 12,897298 & 0,060035 & 0,015931 \\
$\mathbf{2}$ & 12,759261 & 0,000013 & 0,000001 \\
\hline
\end{tabular}

TABELA 34- Convergência da Função Objetivo no método da FLBMP

\begin{tabular}{c|ccc}
\hline Iteração & Função Objetivo (MW) & $\mathbf{M A X}(\Delta \mathbf{P})$ & $\mathbf{M A X}(\Delta \mathbf{Q})$ \\
\hline $\mathbf{0}$ & 12,759261 & 0,000013 & 0,000001 \\
$\mathbf{1}$ & 12,436279 & 0,006992 & 0,000040 \\
$\mathbf{2}$ & 12,676492 & 0,000049 & 0,000003 \\
$\mathbf{3}$ & 12,667172 & 0,000000 & 0,000000 \\
$\mathbf{4}$ & 12,666827 & 0,000000 & 0,000000 \\
\hline
\end{tabular}




\subsubsection{SISTEMA ELÉTRICO DE 5 BARRAS}

O sistema elétrico de 5 barras possui as seguintes características:

- 1 barra de geração (slack);

- 1 barra de controle de reativo;

- 3 barras de carga;

- 7 linhas de transmissão.

As tabelas 35 e 36 mostram o processo de convergência da função objetivo para o método da FLBCP e para o método da FLBMP. Os dados apresentados nestas tabelas não estão em p.u.

TABELA 35 - Convergência da Função Objetivo no método da FLBCP

\begin{tabular}{c|ccc}
\hline Iteração & Função Objetivo (MW) & $\mathbf{M A X}(\Delta \mathbf{P})$ & $\mathbf{M A X}(\Delta \mathbf{Q})$ \\
\hline $\mathbf{0}$ & 0,000000 & 60,000000 & 9,500000 \\
$\mathbf{1}$ & 4,532303 & 0,000008 & 0,000001 \\
$\mathbf{2}$ & 4,515186 & 0,000149 & 0,000012 \\
\hline
\end{tabular}

TABELA 36 - Convergência da Função Objetivo no método da FLBMP

\begin{tabular}{c|ccc}
\hline Iteração & Função Objetivo (MW) & $\mathbf{M A X}(\Delta \mathbf{P})$ & $\mathbf{M A X}(\Delta \mathbf{Q})$ \\
\hline $\mathbf{0}$ & 4,515186 & 0,000149 & 0,000012 \\
$\mathbf{1}$ & 4,458584 & 0,001265 & 0,000173 \\
$\mathbf{2}$ & 4,423342 & 0,001377 & 0,000189 \\
$\mathbf{3}$ & 4,423271 & 0,000000 & 0,000000 \\
$\mathbf{4}$ & 4,423760 & 0,000000 & 0,000000 \\
\hline
\end{tabular}

No estado final do sistema de 5 barras, todas as tensões e potências reativas permaneceram dentro dos seus limites; a solução final obedeceu a todas as restrições do sistema, satisfazendo as condições de KKT com uma precisão de $10^{-5}$ p.u.

\subsubsection{SISTEMA ELÉTRICO DE 14 BARRAS}

O sistema elétrico de 14 barras possui as seguintes características:

- 1 barra de geração (slack);

- 4 barras de controle de reativo; 
- 9 barras de carga;

- 17 linhas de transmissão.

As tabelas 37 e 38 mostram o processo de convergência da função objetivo para o método da FLBCP e para o método da FLBMP. Os dados apresentados nestas tabelas não estão em p.u.

TABELA 37 - Convergência da Função Objetivo no método da FLBCP

\begin{tabular}{c|ccc}
\hline Iteração & Função Objetivo (MW) & MAX $(\Delta \mathbf{P})$ & $\mathbf{M A X}(\Delta \mathbf{Q})$ \\
\hline $\mathbf{0}$ & 13,450398 & 22,375284 & 28,975811 \\
$\mathbf{1}$ & 13,348089 & 0,004954 & 0,000338 \\
$\mathbf{2}$ & 12,967919 & 0,005106 & 0,001156 \\
\hline
\end{tabular}

TABELA 38- Convergência da Função Objetivo no método da FLBMP

\begin{tabular}{c|ccc}
\hline Iteração & Função Objetivo (MW) & $\mathbf{M A X}(\Delta \mathbf{P})$ & $\mathbf{M A X}(\boldsymbol{\Delta Q} \mathbf{Q})$ \\
\hline $\mathbf{0}$ & 12,967919 & 0,005106 & 0,001156 \\
$\mathbf{1}$ & 12,748487 & 0,008531 & 0,001839 \\
$\mathbf{2}$ & 12,526919 & 0,009028 & 0,001665 \\
$\mathbf{3}$ & 12,290513 & 0,000000 & 0,000000 \\
$\mathbf{4}$ & 12,292477 & 0,000000 & 0,000000 \\
$\mathbf{5}$ & 12,292409 & 0,000000 & 0,000000 \\
$\mathbf{6}$ & 12,292406 & 0,000000 & 0,000000 \\
\hline
\end{tabular}

No estado final do sistema de 14 barras, todas as tensões e potências reativas permaneceram dentro dos seus limites; a solução final obedeceu a todas as restrições do sistema, satisfazendo as condições de KKT com uma precisão de $10^{-5}$ p.u.

\subsubsection{SISTEMA ELÉTRICO DE 30 BARRAS}

O sistema elétrico de 30 barras possui as seguintes características:

- 1 barra de geração (slack);

- 5 barras de controle de reativo;

- 24 barras de carga;

- 37 linhas de transmissão. 
As tabelas 39 e 40 mostram o processo de convergência da função objetivo para o método da FLBCP e para o método da FLBMP. Os dados apresentados nestas tabelas não estão em p.u.

TABELA 39 - Convergência da Função Objetivo no método da FLBCP

\begin{tabular}{c|ccc}
\hline Iteração & Função Objetivo (MW) & $\mathbf{M A X}(\Delta \mathbf{P})$ & $\mathbf{M A X}(\Delta \mathbf{Q})$ \\
\hline $\mathbf{0}$ & 1,442716 & 93,605417 & 33,685585 \\
$\mathbf{1}$ & 16,803827 & 0,001127 & 0,000119 \\
$\mathbf{2}$ & 16,460361 & 0,000628 & 0,000104 \\
\hline
\end{tabular}

TABELA 40 - Convergência da Função Objetivo no método da FLBMP

\begin{tabular}{c|ccc}
\hline Iteração & Função Objetivo $(\mathbf{M W})$ & $\mathbf{M A X}(\Delta \mathbf{P})$ & $\mathbf{M A X}(\Delta \mathbf{Q})$ \\
\hline $\mathbf{0}$ & 16,460361 & 0,000628 & 0,000104 \\
$\mathbf{1}$ & 16,223415 & 0,003025 & 0,000542 \\
$\mathbf{2}$ & 16,177369 & 0,002664 & 0,000889 \\
$\mathbf{3}$ & 16,195834 & 0,000021 & 0,000003 \\
$\mathbf{4}$ & 16,192573 & 0,000000 & 0,000000 \\
$\mathbf{5}$ & 16,190650 & 0,000000 & 0,000000 \\
\hline
\end{tabular}

No estado final do sistema de 30 barras, todas as tensões e potências reativas permaneceram dentro dos seus limites; a solução final obedeceu a todas as restrições do sistema, satisfazendo as condições de KKT com uma precisão de $10^{-5}$ p.u.

\subsubsection{SISTEMA ELÉTRICO DE 57 BARRAS}

O sistema elétrico de 57 barras possui as seguintes características:

- 1 barra de geração (slack);

- 6 barras de controle de reativo;

- 50 barras de carga;

- 80 linhas de transmissão.

As tabelas 41 e 42 mostram o processo de convergência da função objetivo para o método da FLBCP e para o método da FLBMP. Os dados apresentados nestas tabelas não estão em p.u. 
TABELA 41 - Convergência da Função Objetivo no método da FLBCP

\begin{tabular}{c|ccc}
\hline Iteração & Função Objetivo (MW) & $\mathbf{M A X}(\Delta \mathbf{P})$ & $\mathbf{M A X}(\Delta \mathbf{Q})$ \\
\hline $\mathbf{0}$ & 3,467398 & 286,539656 & 47,465987 \\
$\mathbf{1}$ & 27,180882 & 0,027288 & 0,006041 \\
$\mathbf{2}$ & 25,770103 & 0,023872 & 0,003278 \\
\hline
\end{tabular}

TABELA 42 - Convergência da Função Objetivo no método da FLBMP

\begin{tabular}{c|ccc}
\hline Iteração & Função Objetivo $(\mathbf{M W})$ & $\mathbf{M A X}(\boldsymbol{\Delta P})$ & $\mathbf{M A X}(\boldsymbol{\Delta Q})$ \\
\hline $\mathbf{0}$ & 25,770103 & 0,023872 & 0,003278 \\
$\mathbf{1}$ & 25,414650 & 0,023535 & 0,003221 \\
$\mathbf{2}$ & 24,272139 & 0,024459 & 0,003560 \\
$\mathbf{3}$ & 23,022722 & 0,042244 & 0,002193 \\
$\mathbf{4}$ & 23,464630 & 0,003521 & 0,000608 \\
$\mathbf{5}$ & 23,376332 & 0,001520 & 0,000238 \\
$\mathbf{6}$ & 23,352551 & 0,000000 & 0,000000 \\
$\mathbf{7}$ & 23,343054 & 0,000032 & 0,000005 \\
$\mathbf{8}$ & 23,331434 & 0,000052 & 0,000007 \\
$\mathbf{9}$ & 23,263312 & 0,000000 & 0,000000 \\
\hline
\end{tabular}

No estado final do sistema de 57 barras, todas as tensões e potências reativas permaneceram dentro dos seus limites; a solução final obedeceu a todas as restrições do sistema, satisfazendo as condições de KKT com uma precisão de $10^{-5}$ p.u.

\subsubsection{SISTEMA ELÉTRICO DE 118 BARRAS}

O sistema elétrico de 118 barras possui as seguintes características:

- 1 barra de geração (slack);

- 51 barras de controle de reativo;

- 66 barras de carga;

- 186 linhas de transmissão.

As tabelas 43 e 44 mostram o processo de convergência da função objetivo para o método da FLBCP e para o método da FLBMP. Os dados apresentados nestas tabelas não estão em p.u. 
TABELA 43 - Convergência da Função Objetivo no método da FLBCP

\begin{tabular}{c|ccc}
\hline Iteração & Função Objetivo (MW) & $\mathbf{M A X}(\Delta \mathbf{P})$ & $\mathbf{M A X}(\Delta \mathbf{Q})$ \\
\hline $\mathbf{0}$ & 0,000000 & 607,000000 & 119,810000 \\
$\mathbf{1}$ & 117,736533 & 0,001318 & 0,000034 \\
$\mathbf{2}$ & 117,224971 & 0,001821 & 0,000057 \\
\hline
\end{tabular}

TABELA 44 - Convergência da Função Objetivo no método da FLBMP

\begin{tabular}{c|ccc}
\hline Iteração & Função Objetivo $(\mathbf{M W})$ & $\mathbf{M A X}(\boldsymbol{\Delta} \mathbf{P})$ & $\mathbf{M A X}(\boldsymbol{\Delta Q})$ \\
\hline $\mathbf{0}$ & 117,224972 & 0,001821 & 0,000057 \\
$\mathbf{1}$ & 116,811294 & 0,003798 & 0,000210 \\
$\mathbf{2}$ & 116,420025 & 0,005650 & 0,000347 \\
$\mathbf{3}$ & 116,030207 & 0,007412 & 0,000497 \\
\hline
\end{tabular}

No estado final do sistema de 118 barras, todas as tensões e potências reativas permaneceram dentro dos seus limites; a solução final obedeceu a todas as restrições do sistema, satisfazendo as condições de KKT com uma precisão de $10^{-5}$ p.u.

No Apêndice 2 é mostrado o banco de dados dos valores dos sistemas de 3 e 118 barras. O Apêndice 3 fornece o estado final dos sistemas de 3 e 118 barras.

Nos exemplos apresentados, o método convergiu de forma eficiente e obteve o ótimo do problema em um número de iterações aceitável. Salientamos a eficiência da extrapolação quadrática do método, que foi capaz de atingir o ótimo do problema, satisfazendo todo as restrições, mesmo partindo de um ponto inicial que não satisfaça algumas ou todas restrições. Devido a isso, a escolha do parâmetro de barreira não se torna um fator de sensibilidade do método: em todos os testes numéricos realizados, o parâmetro de barreira sempre iniciou-se com o valor 0,01 e ocorreu a convergência sem ter que mudar o valor inicial deste parâmetro. Este fator torna o método eficiente, pois ele evita o desagradável ajuste das variáveis de estado, das potências e dos parâmetros utilizados no problema por um processo de tentativa e erro.

No próximo capítulo serão apresentadas as conclusões e as perspectivas de continuação deste trabalho. 


\section{CAPÍTULO 6}

\section{CONCLUSÕES}

Neste trabalho propomos uma abordagem para a resolução de problemas gerais de otimização. Essa abordagem é denominada de método da função Lagrangiana barreira modificada/penalidade.

Inicialmente, apresentamos um levantamento bibliográfico para o posicionamento da abordagem apresentada. Uma revisão dos métodos da função barreira clássica e da função barreira modificada, os quais forneceram um suporte teórico para a abordagem proposta e sua aplicação em problemas de FPO. Construímos a função Lagrangiana barreira modificada/penalidade, na qual as restrições canalizadas são tratadas ou pelo método de barreira modificada ou por uma extrapolação quadrática (penalidade). O algoritmo foi baseado na solução de uma seqüência de problemas de minimização irrestrita, parametrizados pela barreira, e pelas estimativas dos multiplicadores de Lagrange, ambos associados com a função barreira modificada. Esse algoritmo envolve iterações internas (Newton) e externas (barreira modificada). A cada iteração externa, os parâmetros de barreira e as estimativas dos multiplicadores de Lagrange da função barreira modificada são atualizados. A iteração interna corresponde a um processo para minimização de um problema irrestrito em que os parâmetros de barreira e as estimativas dos multiplicadores de Lagrange estão fixos. As variáveis 
primais e duais são atualizadas pelos fatores de correção, originados da iteração interna, e pelos passos primais e duais respectivamente, determinados por um processo de busca. Um algoritmo para a inicialização dos multiplicadores de Lagrange foi utilizado, baseado na função Lagrangiana barreira clássica/penalidade.

Aplicamos a abordagem em um exemplo teste teórico e realizamos sua implementação computacional, usando o aplicativo MATLAB ${ }^{\circledR}$. O método mostrou-se eficiente na resolução deste exemplo. Verificamos que o número de iterações do método está diretamente ligado à escolha dos parâmetros iniciais de barreira e ao ajuste das estimativas dos multiplicadores de Lagrange associados às restrições do tipo “ $\geq$ ”.

Nesse momento, este trabalho tem sua contribuição na área de Matemática com o desenvolvimento da abordagem da FLBMP e seu método para a resolução de PPNL restritos, e na Engenharia Elétrica com a resolução eficiente de problemas de FPO, sem o “incômodo” do ajuste das variáveis e parâmetros iniciais.

Os trabalhos futuros são enunciados a seguir:

- Desenvolvimento de um sistema em linguagem C para aplicações no setor energético e em problemas de grande porte;

- Realização de testes computacionais em outros sistemas;

- Estudo do comportamento geométrico do método e análise da convergência;

- Aprimoramento dos critérios de convergência. 


\section{REFERÊNCIAS BIBLIOGRÁFICAS}

AKROTIRIANAKIS, I.; RUSTEM, B. (2005) Convergent Interior-Point Algorithm for Nonlinear Programming, Journal of Optimization Theory and Applications, v. 125, $\mathrm{n}^{\circ}$. 3, p. 497-521.

BAKHTIARI, S.; TITS, A. L. (2003). A Simple Primal-Dual Feasible Interior-Point Method for Nonlinear Programming with Monotone Descent. Computational Optimization and Applications, v. 25, p. 17-38.

BEN-TAL, A., YUZEFOVICH, I., \& ZIBULEVSKY, M. (1992). Penalty/barrier multiplier methods for minmax and constrained smooth convex programs. Technical report \#9/92, Faculty of Industrial Engineering and Management, Technion, Israel Institute of Technology, Haifa 32000, Israel.

BOUKARI, D.; FIACCO, A.V. (1995). Survey of Penalty, Exact-Penalty and Multiplier Methods From 1968 to 1993. Optimization, v. 32, p.301-334.

BREITFELD, M. G., \& SHANNO, D. F. (1993). Computational experience with modified log-barrier functions for nonlinear programming. Technical report RRR 17-93, Rutgers Center for Operations Research and Graduate School of Management, Rutgers University, New Brunswick, NJ 08903, USA.

BREITFELD, M. G., \& SHANNO, D. F. (1994b). Computational experience with penalty-barrier methods for nonlinear programming. Technical report RRR 17-93 
Revised, Rutgers Center for Operations Research and Graduate School of Management, Rutgers University, New Brunswick, NJ 08903, USA.

BREITFELD, M. G., \& SHANNO, D. F. (1994c). A globally convergent penaltybarrier algorithm for nonlinear programming and its computational performance. Technical report RRR 12-94, Rutgers Center for Operations Research and Graduate School of Management, Rutgers University, New Brunswick, NJ 08903, USA.

BREITFELD, M.G.; SHANNO, D.F. (1996). Computational experience with penaltybarrier methods for nonlinear programming. Annals of Operations Research, v. 62, p. 439-463.

BYRD, R. H.; NOCEDAL, J.; WALTZ, R. A. (2003). Feasible Interior Methods Using Slacks for Nonlinear Optimization. Computational Optimization and Applications, v. 26, p. 35-61.

CARROL, C.W. (1961). The Created Response Surface Thecnique for Optimizing Nonlinear Restrained Systems. Operations Research, v. 9, p. 169-184.

CHEN T. W. C.; VASSILIADIS V. S. (2003). Solution of general nonlinear ptimization problems using the penalty/modified barrier method with the use of exact Hessians. Computer and Chemical Engineering 27, 501-525.

CONN, A.R.; GOULD, N.; TOINT, P.L. (1997). A Globally Convergent Lagrangian Barrier Algorithm for Optimization With General Inequality Constraints and Simple Bounds. Mathematics of Computation, v. 66, n. 217, p. 261 -288.

DOMMEL, H.W.; TINNEY, W.F. ( 1968). Optimal Power Flow Solutions. IEEE Trans. on PAS., v. 87, p. 1866-1876, October.

EL-BAKRY, A.S.; TAPIA, R.A.; TSUCHIYA, T.; ZHANG, Y. (1996). On the Formulation and Theory of the Newton Interior-Point Method for Nonlinear Programming. Journal of Optimization Theory and Applications, v. 89, n.3, p. 507-541. 
FIACCO, A.V.; McCORMICK, G.P. (1968). Nonlinear Programming: Sequential Unconstrained Minimization Techniques. New York, John Wiley \& Sons.

FIACCO, A.V.; McCORMICK, G.P. (1990). Nonlinear Programming-Sequential Unconstrained Optimization Techniques, Philadelphia, SIAM. (Classics in Applied Mathematics, v. 4).

FORSGREN, A.; Gill, P. E.; Wright, M. H. (2002). Interior Methods for Nonlinear Optimization. SIAM REVIEW, v. 44, n. 4, p. 525-597.

FRERIS, L.L. (1968). Investigation of the Load-Flow Problem. Proc. IEE, v. 115, $\mathrm{n}^{\mathrm{o}}$ 10, p. $1459-1470$.

FRISCH, K.R. (1955). The logarithmic Potential Method of Convex Programming, Memorandum, University Institute of Economics, Oslo, Norway.

GILL, P. E., MURRAY, W., \& WRIGHT, M. H. (1981). Practical optimization. New York: Academic Press.

GILL, P.E.; MURRAY, W.; SAUNDERS, M.A.; WRIGHT, M.H. (1986). On Projected Newton Barrier Methods For Linear Programming and an Equivalence to Karmarkar's Projective Method. Mathematical Programming, v. 36, p. 183-209.

GONZAGA, C.C. (1989). Algoritmos de Pontos Interiores para Programação Linear, 69p., Texto de acompanhamento, I EBO - Escola Brasileira de Otimização

GONZAGA, C. C.; CARDIA, M. (2004). Properties of the central points in linear programming Problems. Numerical Algorithms, v. 35, p. 185-204.

GRIVA, I. A. (2004). Numerical experiments with an interior-exterior point method for nonlinear programming. Journal of Computational Optimization and Applications, vol. 29, pp. 173-195. 
KARMARKAR, N. (1984). A New Polynomial-Time Algorithm for Linear Programming. Combinatorica 4, v. 4, p. 373-395.

KOCVARA, M.; STINGL, M. (2007) On the solution of large-scale SPD problems by modified barrier method using iterative solvers. Math. Program. Ser. B. 109, p. 413 444.

MATUMOTO, L.T.(1996). Algoritmos de Pontos Interiores Para Programação Linear e uma Extensão para a Programação Linear Por Partes. São Carlos. 56p. Dissertação (Mestrado) - Instituto de Ciências Matemáticas e de Computação - Universidade de São Paulo.

MEHROTRA, S. (1992). On implementation of a primal-dual interior point method. SIAM Journal on Optimization, v.2, p. 575-601.

MELMAN, A. (1996). Linesearch procedure in barrier methods for some convex programming problems. SIAM Journal on Optimization, v. 6, n. 2, p. 283-298.

MURRAY, W. (1971). Analytical Expressions for the Eigenvalues and Eigenvectors of the Hessian Matrices of Barrier and Penalty Functions. Journal of Optimization Theory and Applications,v. 7, n. 3, p. 189-196.

NASH, S.G.; POLYAK, R.A.; SOFER A. (1994). A numerical Comparison of Barrier and Modified-Barrier Methods for Large-Scale Bound-Constrained Optimization. Large Scale Optimization: State of the Art, p. 319-338.

NOCEDAL, J.; WRIGHT, S. (1999). Numerical Optimization. New York: SpringerVerlag.

POLYAK, R.A. (1992). Modified barrier functions. Mathematical Programming, v. 54, n. 2, p. $177-222$. 
SHANNO, D.F.; VANDERBEI, R.J. (2000). Interior-point methods for nonconvex nonlinear programming: orderings and higher-order methods. Mathematical Programming, Ser. B, v. 87, p. 303-316.

SOUSA, V. A.; COSTA, G. R. M.; BAPTISTA, E. C (2006). A Função Barreira Modificada e o Problema de Fluxo de Potência Ótimo. TEMA - Tendências em Matemática Aplicada e Computacional, v. 7, n. 2, p. 21-30.

TORRES, G.L.; QUINTANA, V.H. (1998). An Interior Point Method for Nonlinear Optimal Power Flow Using Voltage Rectangular Coordinates. IEEE Transactions on Power Systems, v. 13, nº 4, p. 1211-1218, November.

VANDERBEI, R. J.; SHANNO, D. F. (1999). An Interior-Point Algorithm for Nonconvex Nonlinear Programming. Computational Optimization and Applications, v. 13, p. 231-252.

VASSILIADIS, V. S., \& BROOKS, S. A. (1998). Application of the modified barrier method in large-scale quadratic programming problems. Computer and Chemical Engineering 22, 1197-1205.

WRIGHT, M. H. (1994). Some properties of the Hessian of the logarithmic barrier function. Mathematical Programming, v. 67, p. 265-295.

WRIGHT, M. H. (1995). Why a pure primal Newton barrier step may be infeasible. SIAM Journal on Optimization, v. 5, n 1, p. 1-12.

WRIGHT. S.; JARRE, F. (1999). The role of linear objective functions in barrier methods. Mathematical programming, v. 84, p. 357-373. 


\section{LEITURAS COMPLEMENTARES}

BAZARAA, M.S.; SHERALI, H.D.; SHETTY, C.M. (1993). Nonlinear Programming: Theory and Algorithms. 2.ed. New York, John Wiley.

BREITFELD, M. G., SHANNO, D. F. (1994a). Preliminary computational experience with modified log-barrier functions for large-scale nonlinear programming. In $W$. $W$. Hager, D. W. Hearn \& P. M. Pardalos (Eds.), Large scale optimization: state of the art (pp. 45_67). Kluwer Academic Publishers.

BREITFELD, M.G.; SHANNO, D. F. (1996). Computational experience with penaltybarrier methods for nonlinear programming. Annals of Operations Research, v. 62, p. 439-463.

LUENBERGER, D.G. (1984). Linear and Nonlinear Programming. Addison-Wesley Publishing Company Inc., Second Edition. 


\section{APÊNDICE 1}

\section{ALGORITMOS ESTRUTURADOS}

\section{ALGORITMO FLBCP - INICIALIZAÇÃO DO ALGORITMO FLBMP}

\section{$\rightarrow \underline{\text { Início do Ciclo Externo da FLBCP }}$}

i. Determinar uma solução inicial $X^{0}=\left(x_{0}, z_{0}, \lambda_{0}{ }^{E}, \lambda_{0}{ }^{\mathrm{I}}\right)$.

ii. Determinar a FLBCP (4.58) associada ao problema.

iii. Faça CONT = 1 (contador das iterações do ciclo externo do método da FLBCP) e enquanto CONT $\leq$ Nitemax (número máximo de iterações, que no caso será 2), faça

iii.1. Ajuste $\alpha_{0}=1$ e avalie $G_{F L B C P}$.

\section{$\rightarrow$ Início do Ciclo Interno da FLBCP}

iii.2. Faça CONTINT $=1$ (Contador das iterações do ciclo interno do método da FLBCP. Enquanto $\left\|G_{\text {FLBCP }}\left(\mathrm{x}_{0}, \mathrm{z}_{0}, \lambda_{0}^{\mathrm{E}}, \lambda_{0}^{\mathrm{I}}\right)\right\|>\varepsilon$ faça (ciclo interno do método da FLBCP)

iii.2.1. Avalie a Hessiana $\mathrm{H}_{\mathrm{FLBCP}}$. Calcule a direção de busca $S$, resolvendo o sistema (4.69) e calcule $X^{\mathrm{p}}$, na forma de (4.32), usando (4.31).

iii.2.2. Atualize $M$ por (4.39) e avalie $\mathrm{FPM}_{\mathrm{b}}(4.72)$ em $\mathrm{X}^{0}$ e $\mathrm{X}^{\mathrm{P}}$.

iii.2.3. Faça NiteBL $=1$ (contador das iterações da busca linear) e enquanto (4.33) não é satisfeita (Ciclo da Busca Linear) faça

\section{$\rightarrow \underline{\text { Início da Busca Linear }}$}

iii.2.3.1. Atualize $\alpha$ por (4.34), as variáveis primais por (4.35) e as 
duais por (4.36) e (4.37). Faça $\left(\lambda^{\mathrm{E}}\right)^{\mathrm{BL}}=\left(\lambda^{\mathrm{E}}\right)^{\mathrm{BL}+1}$ e $\left(\lambda^{\mathrm{I}}\right)^{\mathrm{BL}}=\left(\lambda^{\mathrm{I}}\right)^{\mathrm{BL}+1}$

iii.2.3.2. Atualize os parâmetros da extrapolação e o parâmetro M. Avalie a FLBCP em $X^{\mathrm{BL}+1}$. Se (4.33) não for satisfeita, faça NiteBL $=$ NiteBL +1 e volte em iii.2.3.1. Caso contrário, fim da busca linear.

\section{$\rightarrow \underline{\text { Fim da Busca Linear }}$}

iii.2.4. Faça $X^{0}=X^{B L}$. Se $\left\|G_{F L B C P}\left(x_{0}, z_{0}, \lambda_{0}^{E}, \lambda_{0}^{I}\right)\right\| \leq \varepsilon$, fim do ciclo interno. Caso contrário, faça CONTINT = CONTINT + 1 volte em iii 2.1.

\section{$\rightarrow$ Fim do Ciclo Interno}

iii.3. Se CONT $\leq$ Nitemax atualize $\mu$ por (4.39), faça $X^{0}=X^{p}$ e volte para iii.1. Caso contrário, faça $\mathrm{X}^{0}=\mathrm{X}^{\mathrm{p}}$ e saia do ciclo externo.

\section{$\rightarrow$ Fim do Ciclo Externo}

iv. Calcule v ${ }^{\mathrm{I}}, \mathrm{v}^{\mathrm{S}}, \xi^{\mathrm{I}}, \xi^{\mathrm{S}}$ usando (4.56).

\section{ALGORITMO DA FLBMP}

i. Execute o algoritmo da FLBCP para obter uma solução inicial e uma estimativa para os multiplicadores de Lagrange associados as restrições de desigualdade.

ii. Determine a FLBMP (4.17) associada ao problema. Se (4.73) ou (4.74) são satisfeitos, a solução inicial é ótima e fim. Caso contrário, entrar no ciclo externo.

\section{$\rightarrow$ Início do Ciclo Externo da FLBMP}

iii. Faça CONT = 1 (contador das iterações do ciclo externo do método da FLBMP) e enquanto (CONT $\leq$ Nitemax ou (4.72) não é satisfeito ou (4.73) não é satisfeito), faça

iii.1. Ajuste $\alpha_{0}=1$ e avalie $G_{\text {FLBMP. }}$.

\section{$\rightarrow \underline{\text { Início do Ciclo Interno da FLBMP }}$}

iii.2. Faça CONTINT $=1$ (Contador das iterações do ciclo interno do método da FLBMP. Enquanto (4.40) não é satisfeita, faça

iii.2.1. Avalie a Hessiana $\mathrm{H}_{\mathrm{FLBmp}}$. Calcule a direção de busca $S$, resolvendo 
o sistema (4.20) e calcule $\mathrm{X}^{\mathrm{p}}$, na forma de (4.32), usando (4.31).

iii.2.2. Atualize $M$ por (4.39) e avalie $\mathrm{FPM}_{\mathrm{a}}$ (4.38) em $\mathrm{X}^{0}$ e $\mathrm{X}^{\mathrm{p}}$.

iii.2.3. Faça NiteBL $=1$ (contador das iterações da busca linear) e enquanto (4.33) não é satisfeita

\section{$\rightarrow \underline{\text { Início da Busca Linear }}$}

iii.2.3.1. Atualize $\alpha$ por (4.34), as variáveis primais por (4.35) e as duas por (4.36) e (4.37). Faça $\left(\lambda^{\mathrm{E}}\right)^{\mathrm{BL}}=\left(\lambda^{\mathrm{E}}\right)^{\mathrm{BL}+1}$ e $\left(\lambda^{\mathrm{I}}\right)^{\mathrm{BL}}=\left(\lambda^{\mathrm{I}}\right)^{\mathrm{BL}+1}$

iii.2.3.2. Atualize os parâmetros da extrapolação e o parâmetro M. Avalie a $\mathrm{FPM}_{\mathrm{a}}$ em $\mathrm{X}^{\mathrm{BL}+1}$. Se (4.33) não for satisfeita, faça NiteBL $=$ NiteBL +1 e volte em iii.2.3.1. Caso contrário, fim da busca linear.

\section{$\rightarrow$ Fim da Busca Linear}

iii.2.4. Faça $X^{0}=X^{B L}$. Se (4.40) for satisfeita, fim do ciclo interno. Caso contrário, faça CONTINT $=$ CONTINT +1 volte em iii.2.1.

\section{$\rightarrow \underline{\text { Fim do Ciclo Interno }}$}

iii.3. Se (CONT > Nitemax ou (4.73) é satisfeita ou (4.74) é satisfeita), faça $\mathrm{X}^{0}=\mathrm{X}^{\mathrm{p}}$ e saia do ciclo externo. Caso contrário, atualize $\mu$ por (4.39), faça $\mathrm{X}^{0}=\mathrm{X}^{\mathrm{p}}$ e volte para iii.1.

\section{$\rightarrow$ Fim do Ciclo Externo}

iv. Mostrar a solução ótima obtida $\mathrm{X}^{0}$. 


\title{
APÊNDICE 2
}

\section{BANCO DE DADOS DOS SISTEMAS ELÉTRICOS}

\author{
Sistema Elétrico de 3 barras
}

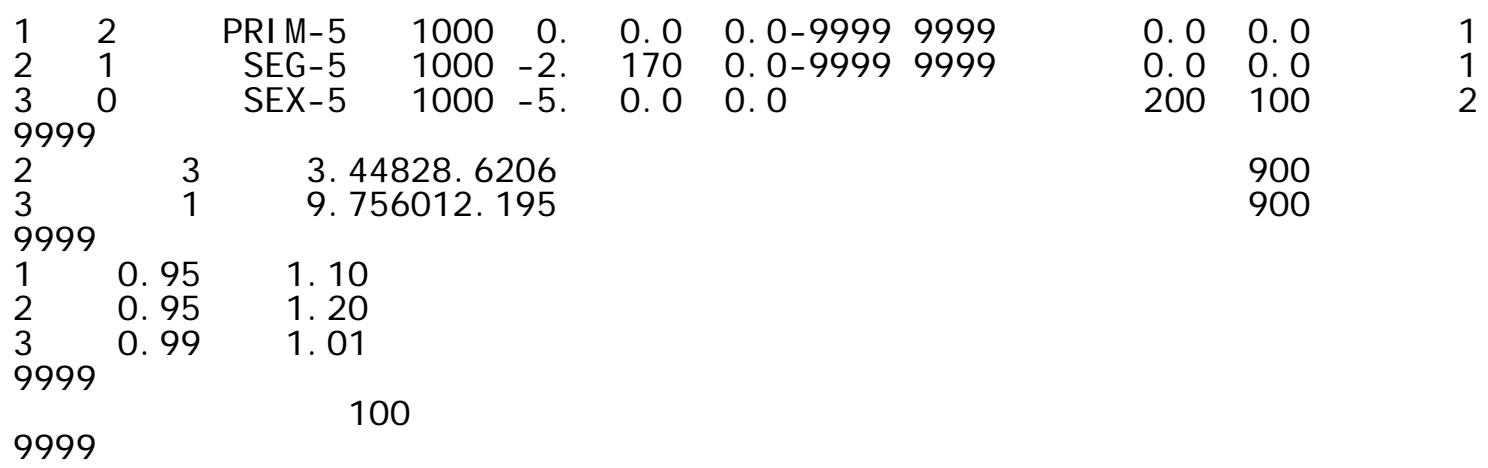

Sistema Elétrico de 118 barras

\begin{tabular}{|c|c|c|c|c|c|c|}
\hline $\begin{array}{r}12 \\
2 \\
3 \\
4 \\
5 \\
6 \\
7 \\
8 \\
9 \\
10 \\
11 \\
1 \\
13 \\
14 \\
15 \\
16 \\
17 \\
18\end{array}$ & $\begin{array}{ll}2 & \text { BARRA12 } \\
0 & \text { BARRA2 } \\
0 & \text { BARRA3 } \\
1 & \text { BARRA4 } \\
0 & \text { BARRA5 } \\
1 & \text { BARRA6 } \\
0 & \text { BARRA7 } \\
1 & \text { BARRA8 } \\
0 & \text { BARRA9 } \\
1 & \text { BARRA10 } \\
0 & \text { BARRA11 } \\
1 & \text { BARRA1 } \\
0 & \text { BARRA13 } \\
0 & \text { BARRA14 } \\
1 & \text { BARRA15 } \\
0 & \text { BARRA16 } \\
0 & \text { BARRA17 } \\
1 & \text { BARRA18 }\end{array}$ & $\begin{array}{l}1000 \\
1000 \\
1000 \\
1000 \\
1000 \\
1000 \\
1000 \\
1000 \\
1000 \\
1000 \\
1000 \\
1000 \\
1000 \\
1000 \\
1000 \\
1000 \\
1000 \\
1000\end{array}$ & $\begin{array}{l}.0 \\
.0 \\
.0 \\
.0 \\
.0 \\
.0 \\
0 \\
.0 \\
.0 \\
.0 \\
.0 \\
0 \\
.0 \\
.0 \\
.0 \\
.0 \\
.0 \\
.0\end{array}$ & $\begin{array}{l}-50 . \\
-50 \\
-50 \\
-250 \\
-50 \\
-50 \\
-50\end{array}$ & $\begin{array}{c}50 . \\
50 . \\
50 . \\
250 . \\
50 . \\
50 . \\
50 .\end{array}$ & $\begin{array}{rr}.0 & .0 \\
20.0 & 9.0 \\
39.0 & 10.0 \\
39.0 & 12.0 \\
.0 & .0 \\
52.0 & 22.0 \\
19.0 & 2.0 \\
28.0 & 0.0 \\
.0 & .0 \\
-450 . & .0 \\
70.0 & 23.0 \\
51.0 & 27.0 \\
34.0 & 16.0 \\
14.0 & 1.0 \\
90.0 & 30.0 \\
25.0 & 10.0 \\
11.0 & 3.0 \\
60.0 & 34.0\end{array}$ \\
\hline
\end{tabular}




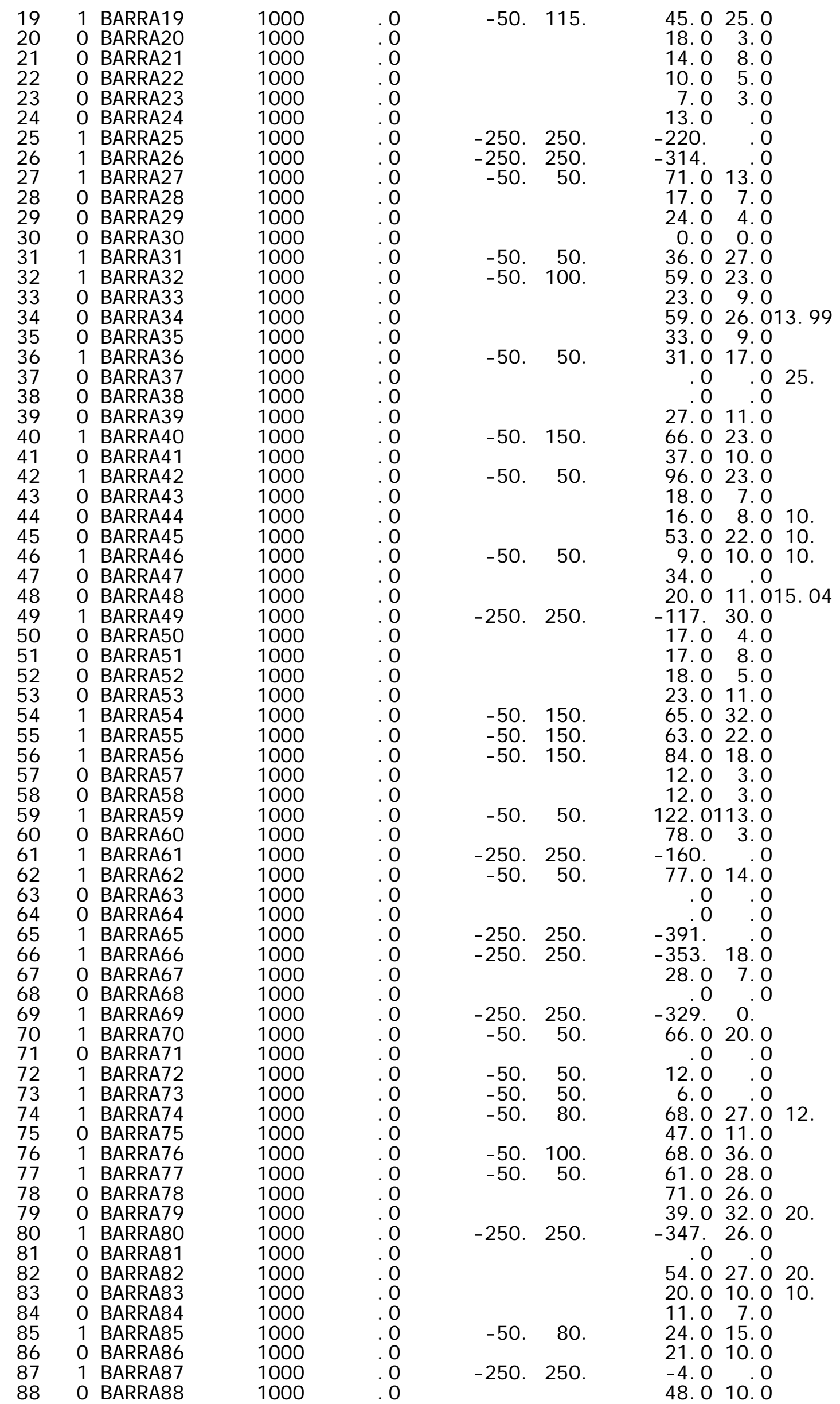




\begin{tabular}{|c|c|c|c|c|c|c|c|c|c|}
\hline $\begin{array}{r}89 \\
90 \\
91 \\
92 \\
93 \\
94 \\
95 \\
96 \\
97 \\
98 \\
99 \\
100 \\
101 \\
102 \\
103 \\
104 \\
105 \\
106 \\
107 \\
108 \\
109 \\
110 \\
111 \\
112 \\
113 \\
114 \\
115 \\
116 \\
117 \\
118\end{array}$ & 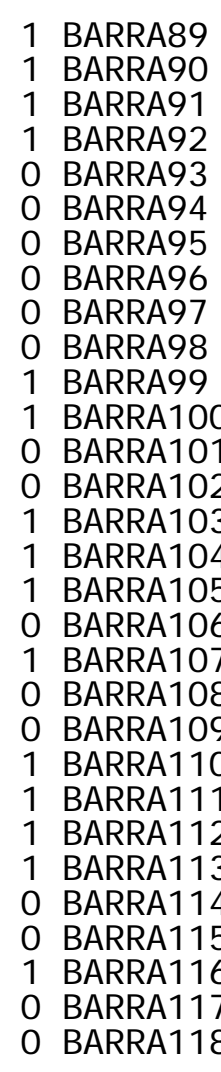 & & 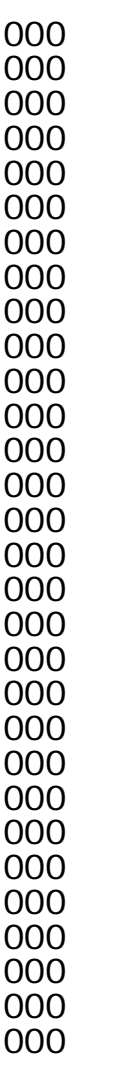 & $\begin{array}{l}.0 \\
.0 \\
.0 \\
.0 \\
.0 \\
.0 \\
.0 \\
.0 \\
.0 \\
.0 \\
.0 \\
.0 \\
.0 \\
0 \\
0 \\
0 \\
0 \\
.0 \\
.0 \\
0 \\
.0 \\
.0 \\
.0 \\
.0 \\
.0 \\
.0 \\
.0 \\
.0 \\
.0 \\
.0\end{array}$ & & $\begin{array}{r}-50 . \\
-250 . \\
-250 \\
-50 \\
-50 \\
-50\end{array}$ & $\begin{array}{r}250 . \\
0 . \\
20 . \\
60 . \\
\\
0 . \\
0 . \\
50 . \\
0 . \\
\\
0 .\end{array}$ & $\begin{array}{r}-607 . \\
163.0 \\
10.0 \\
65.0 \\
12.0 \\
30.0 \\
42.0 \\
38.0 \\
15.0 \\
34.0 \\
42.0 \\
-215 . \\
22.0 \\
5.0 \\
-17.0 \\
38.0 \\
31.0 \\
43.0 \\
50.0 \\
2.0 \\
8.0 \\
39.0 \\
-36.0 \\
68.0 \\
6.0 \\
8.0 \\
22.0 \\
0.0 \\
20.0 \\
33.0\end{array}$ & $\begin{array}{r}\text { 42.0 } \\
.0 \\
10.0 \\
7.0 \\
16.0 \\
31.0 \\
15.0 \\
9.0 \\
8.0 \\
18.0 \\
15.0 \\
3.0 \\
16.0 \\
25.0 \\
26.0 \\
16.0 \\
12.0 \\
1.0 \\
3.0 \\
30.0 \\
13.0 \\
13.0 \\
3.0 \\
3.0 \\
7.0 \\
8.0 \\
15.0 \\
15.0\end{array}$ \\
\hline $\begin{array}{r}1 \\
1 \\
4 \\
3 \\
5 \\
6 \\
8 \\
8 \\
9 \\
4 \\
5 \\
11 \\
2 \\
3 \\
7 \\
11 \\
12 \\
13 \\
14 \\
12 \\
15 \\
16 \\
17 \\
18 \\
19 \\
15 \\
20 \\
21 \\
22 \\
23 \\
23 \\
26 \\
25 \\
27 \\
28 \\
30 \\
8 \\
26 \\
17\end{array}$ & $\begin{array}{r}2 \\
3 \\
5 \\
5 \\
6 \\
7 \\
9 \\
5 \\
10 \\
11 \\
11 \\
12 \\
12 \\
12 \\
12 \\
13 \\
14 \\
15 \\
15 \\
16 \\
17 \\
17 \\
18 \\
19 \\
20 \\
19 \\
21 \\
22 \\
23 \\
24 \\
25 \\
25 \\
27 \\
28 \\
29 \\
17 \\
30 \\
30 \\
31\end{array}$ & $\begin{array}{l}\text { 3. } 03 \\
1.29 \\
0.176 \\
2.41 \\
1.19 \\
0.459 \\
0.244 \\
0.00 \\
0.258 \\
2.09 \\
2.03 \\
0.595 \\
1.87 \\
4.84 \\
0.862 \\
2.225 \\
2.15 \\
7.442 \\
5.951 \\
2.12 \\
1.32 \\
4.541 \\
1.23 \\
1.119 \\
2.521 \\
1.20 \\
1.83 \\
2.09 \\
3.421 \\
1.35 \\
1.56 \\
0.00 \\
3.181 \\
1.913 \\
2.37 \\
0.00 \\
.431 \\
.799 \\
4.741\end{array}$ & $\begin{array}{r}9.99 \\
4.24 \\
0.798 \\
10.80 \\
5.40 \\
2.08 \\
3.05 \\
2.67 \\
3.22 \\
6.88 \\
6.82 \\
1.96 \\
6.16 \\
16.00 \\
3.40 \\
7.31 \\
7.07 \\
24.44 \\
19.50 \\
8.34 \\
4.37 \\
18.01 \\
5.05 \\
4.93 \\
11.70 \\
3.94 \\
8.49 \\
9.70 \\
15.90 \\
4.92 \\
8.00 \\
3.82 \\
16.30 \\
8.55 \\
9.43 \\
3.88 \\
5.04 \\
8.60 \\
15.63\end{array}$ & $\begin{array}{r}2.540 \\
1.082 \\
0.210 \\
2.840 \\
1.426 \\
0.550 \\
116.62 \\
0.000 \\
123.00 \\
1.748 \\
1.738 \\
0.502 \\
1.572 \\
4.060 \\
0.874 \\
1.876 \\
1.816 \\
6.268 \\
5.020 \\
2.140 \\
4.440 \\
4.660 \\
1.298 \\
1.142 \\
2.980 \\
1.010 \\
2.160 \\
2.460 \\
4.040 \\
4.980 \\
8.640 \\
0.000 \\
17.640 \\
2.160 \\
2.380 \\
0.000 \\
51.400 \\
91.800 \\
3.990\end{array}$ & . & .95 & 1. 05 & $\begin{array}{l}900 . \\
900 . \\
900 . \\
900 . \\
900 . \\
900 . \\
900 . \\
900 . \\
900 . \\
900 . \\
900 . \\
900 . \\
900 . \\
900 . \\
900 . \\
900 . \\
900 . \\
900 . \\
900 . \\
900 . \\
900 . \\
900 . \\
900 . \\
900 . \\
900 . \\
900 . \\
900 . \\
900 . \\
900 . \\
900 . \\
900 . \\
900 . \\
900 . \\
900 . \\
900 . \\
900 . \\
900 . \\
900 . \\
900 .\end{array}$ & $\begin{array}{l}900.0 \\
900.0 \\
900.0 \\
900.0 \\
900.0 \\
900.0 \\
900.0 \\
900.0 \\
900.0 \\
900.0 \\
900.0 \\
900.0 \\
900.0 \\
900.0 \\
900.0 \\
900.0 \\
900.0 \\
900.0 \\
900.0 \\
900.0 \\
900.0 \\
900.0 \\
900.0 \\
900.0 \\
900.0 \\
900.0 \\
900.0 \\
900.0 \\
900.0 \\
900.0 \\
900.0 \\
900.0 \\
900.0 \\
900.0 \\
900.0 \\
900.0 \\
900.0 \\
900.0 \\
900.0\end{array}$ \\
\hline
\end{tabular}




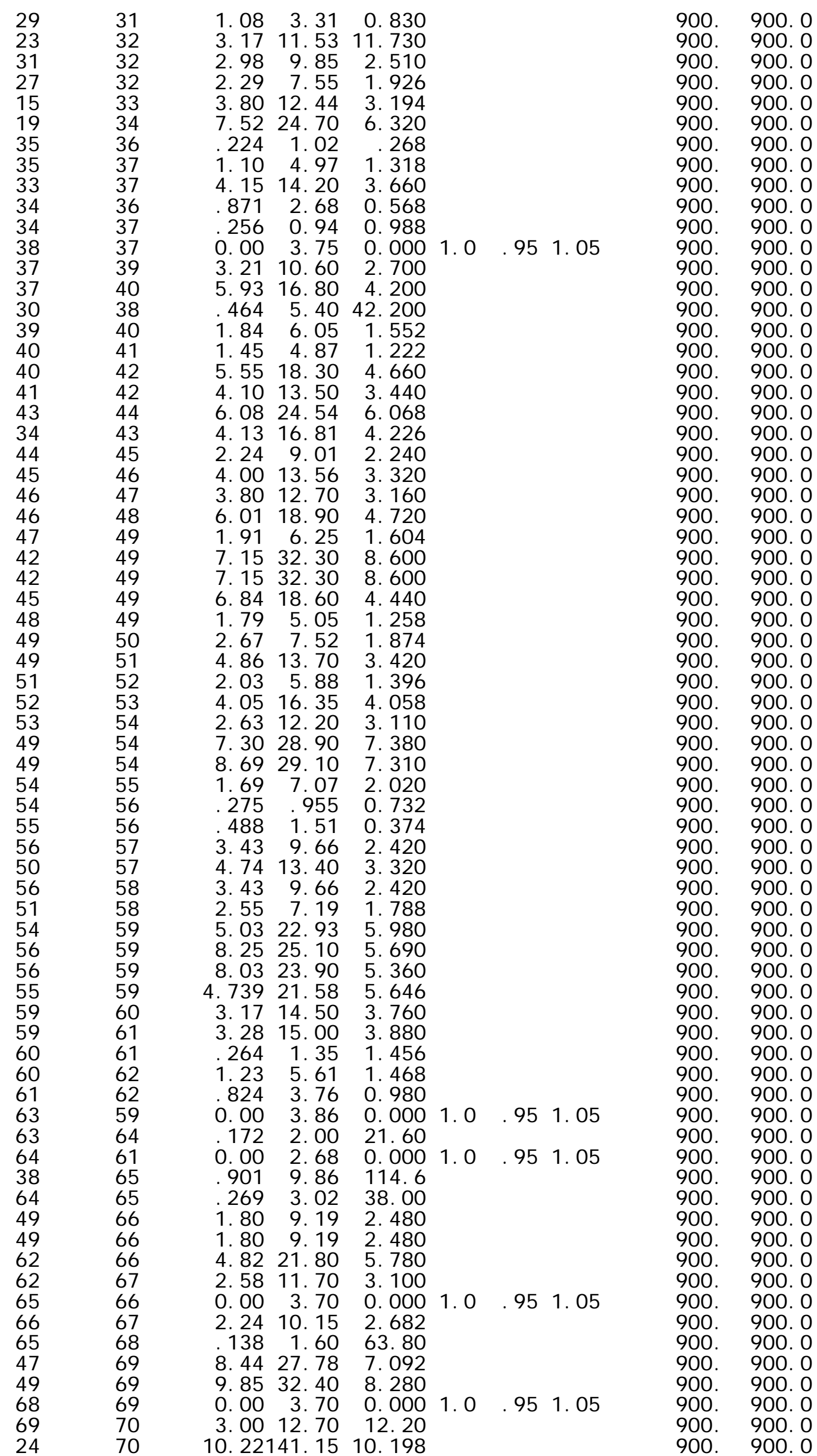




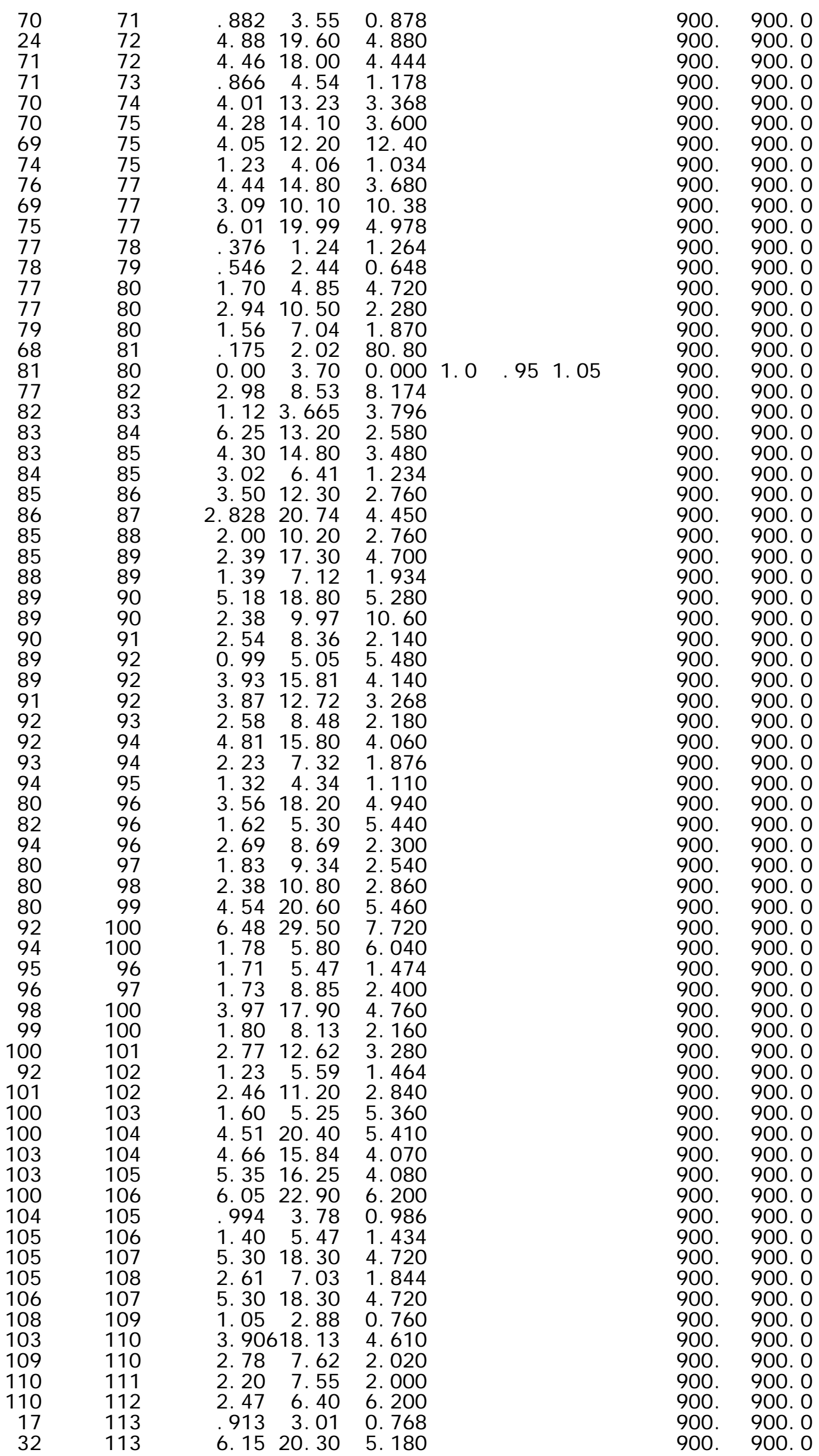




$\begin{array}{rrrrr}32 & 114 & 1.35 & 6.12 & 1.628 \\ 27 & 115 & 1.64 & 7.41 & 1.972 \\ 114 & 115 & 0.23 & 1.04 & 0.276 \\ 68 & 116 & .034 & .405 & 16.40 \\ 12 & 117 & 3.29 & 14.00 & 3.580 \\ 75 & 118 & 1.45 & 4.81 & 1.198 \\ 76 & 118 & 1.64 & 5.44 & 1.356 \\ 9999 & 0.95 & 1.1 & & \\ 9999 & & 900 & & \\ 9999 & & & \end{array}$

900. 900. 0

900. 900. 0

900. 900.0

900. 900. 0

900. 900. 0

900. 900.0

900. 900. 0 


\section{APÊNDICE 3}

\section{ESTADO FINAL DOS SISTEMAS ELÉTRICOS}

\section{Sistema Elétrico de 3 barras}

ESTADO FINAL DO SISTEMA DE 3 BARRAS

NUMERO DE | TERAÇOES $=4$

PARAMETRO DE BARREIRA FINAL $=0.00001000000000000000$

TENSOES E ANGULOS FINAIS DO SISTEMA

$\begin{array}{clc}\text { BARRA } & \text { TENSAO } & \text { ANGULO (em radianos) } \\ 1 & 1.080253 & 0.000000 \\ 2 & 1.133406 & 0.075505 \\ 3 & 1.010000 & -0.022367\end{array}$

VARIAVEIS DE FOLGA

BARRA Z Z

$2 \quad 1.006054$

MULTIPLICADORES DE LAGRANGE DAS RESTRIÇOES DE I GUALDADE DELTA P

$\begin{array}{cl}\text { BARRA } & \text { LAMB E } \\ 2 & 0.027468 \\ 3 & -0.077417\end{array}$


MULTIPLICADORES DE LAGRANGE DAS RESTRIÇOES DE I GUALDADE DELTA Q BARRA LAMB E

$3 \quad-0.04 \overline{8} 929$

MULTI PLI CADORES DE LAGRANGE DAS RESTRI OES DE I GUALDADE OBTI DAS COM VARIAVEIS DE FOLGA - REATI VOS

$\begin{array}{cl}\text { BARRA } & \text { LAMB I } \\ 2 & 0.000000\end{array}$

MULTIPLI CADORES DE LAGRANGE DAS RESTRI ÇOES DE DESI GUALDADE

$\begin{array}{cll}\text { BARRA } & \text { Vk inf } & \text { Vk sup } \\ 1 & 0.000000 & 0.000000 \\ 2 & 0.000000 & 0.000000 \\ 3 & 0.000000 & 0.246579\end{array}$

MULTIPLI CADORES DE LAGRANGE DAS RESTRI ÇOES DE DESI GUALDADE OBTI DAS COM VARIAVEIS DE FOLGA
BARRA
KSI i nf
KSI sup
2
$0.0 \overline{0} 0000$
$0.0 \overline{0} 0000$

POTENCIA REATI VA GERADA

$\begin{array}{cccc}\text { BARRA } & \text { QMIN } & \text { REATIVO Em MVAr } & \text { QMAX } \\ 1 & -9999.000000 & 28.321263 & 9999.000000 \\ 2 & -9999.000000 & 100.605448 & 9999.000000\end{array}$

TOTAL DE I NJEÇAO DE REATIVO DO SISTEMA $=128.926711 \mathrm{MVAr}$

PERDAS ATIVAS DO SISTEMA $=12.666827 \mathrm{MW}$

EVOLUÇAO DA FUNCAO OBJETIVO, DELTAP E DELTAQ POR I TERAÇAO - FLBCP

$\begin{array}{cccr}\text { I TERAÇAO } & \text { F. O. ( e m MW) } & \text { MAX(DELTAP) } & \text { MAX(DELTAQ) } \\ 0 & 4.140646 & 117.115145 & 159.070224 \\ 1 & 12.897298 & 0.060035 & 0.015931 \\ 2 & 12.759261 & 0.000013 & 0.000001\end{array}$

EVOLUÇAO DA FUNCAO OBJETIVO, DELTAP E DELTAQ POR I TERAÇAO - FLBMP

I TERAÇAO
0
1
2
3
4
F. 0. ( e m MW)
12.759261
12.438279
12.676492
12.667172
MAX ( DELTAP)
0.000013
0.006992
0.000049
0.000000
0.000000
$\operatorname{MAX}(\mathrm{DELTAQ})$
0.000001
0.000040
0.000003
0.000000
0.000000




\section{Sistema Elétrico de 118 barras}

ESTADO FINAL DO SISTEMA DE 118 BARRAS

NUMERO DE I TERAÇOES = 3

PARAMETRO DE BARREIRA FINAL $=0.00010000000000000000$

TENSOES E ANGULOS FINAIS DO SISTEMA

\begin{tabular}{|c|c|c|}
\hline 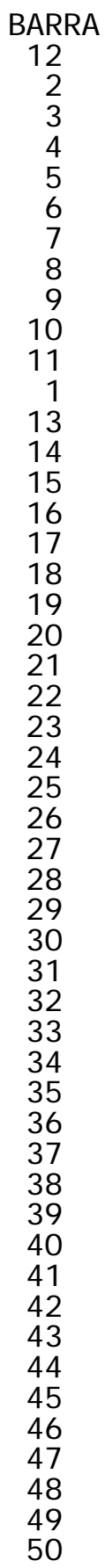 & 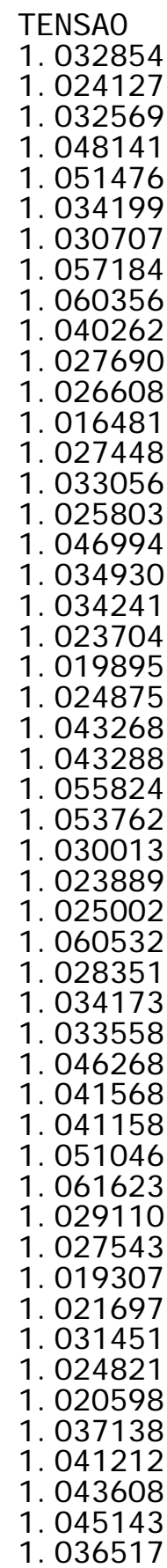 & $\begin{array}{c}\text { ANGULO (em radi anos) } \\
0.000000 \\
-0.017417 \\
-0.001041 \\
0.037153 \\
0.044934 \\
-0.001827 \\
-0.010130 \\
0.127573 \\
0.248906 \\
0.382313 \\
-0.007163 \\
-0.017720 \\
-0.028882 \\
-0.028356 \\
-0.030335 \\
-0.020705 \\
0.012187 \\
-0.024781 \\
-0.034408 \\
-0.021329 \\
0.002882 \\
0.042391 \\
0.120126 \\
0.114347 \\
0.241945 \\
0.271970 \\
0.029896 \\
0.004062 \\
-0.010340 \\
0.096054 \\
-0.008193 \\
0.019571 \\
-0.036140 \\
-0.022376 \\
-0.028888 \\
-0.028915 \\
-0.014596 \\
0.070850 \\
-0.068413 \\
-0.085891 \\
-0.092275 \\
-0.064998 \\
-0.023709 \\
0.015267 \\
0.044987 \\
0.089562 \\
0.125947 \\
0.117510 \\
0.135617 \\
0.099851\end{array}$ \\
\hline
\end{tabular}




\begin{tabular}{|c|c|c|}
\hline $\begin{array}{l}51 \\
52 \\
53 \\
54 \\
55 \\
56 \\
57 \\
58 \\
59 \\
60 \\
61 \\
62 \\
63 \\
64 \\
65 \\
66 \\
67 \\
68 \\
69 \\
70 \\
71 \\
72 \\
73 \\
74 \\
75 \\
76 \\
77 \\
78 \\
79 \\
80 \\
81 \\
82 \\
83 \\
84 \\
85 \\
86 \\
87 \\
88 \\
89 \\
90 \\
91 \\
92 \\
93 \\
94 \\
95 \\
96 \\
97 \\
98 \\
99 \\
100 \\
101 \\
102 \\
103 \\
104 \\
105 \\
106 \\
107 \\
108 \\
109 \\
110 \\
111 \\
112 \\
113 \\
114 \\
115 \\
116 \\
117 \\
118\end{array}$ & $\begin{array}{l}\text { 1. } 021929 \\
1.017040 \\
1.018882 \\
1.035455 \\
1.035492 \\
1.034856 \\
1.032748 \\
1.025356 \\
1.042528 \\
1.036041 \\
1.037649 \\
1.035625 \\
1.045123 \\
1.048224 \\
1.058727 \\
1.051164 \\
1.037920 \\
1.066681 \\
1.053724 \\
1.033268 \\
1.033190 \\
1.034437 \\
1.031464 \\
1.027371 \\
1.025128 \\
1.023033 \\
1.032391 \\
1.027777 \\
1.029182 \\
1.046734 \\
1.065477 \\
1.027553 \\
1.031469\end{array}$ & 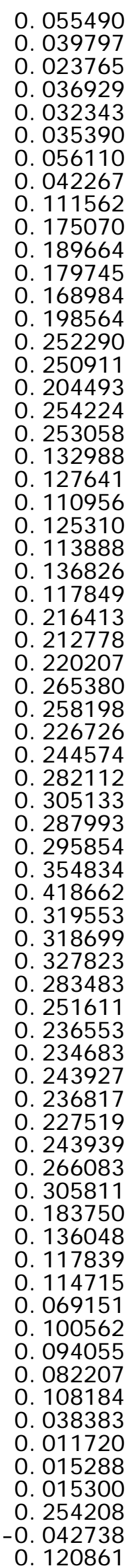 \\
\hline
\end{tabular}


VARIAVEIS DE FOLGA

$\begin{array}{cc}\text { BARRA } & \text { Z } \\ 4 & -0.092100 \\ 6 & -0.029608 \\ 8 & -0.176150 \\ 10 & -1.370857 \\ 12 & 0.022209 \\ 15 & -0.077108 \\ 18 & -0.080694 \\ 19 & 0.113537 \\ 25 & -0.091638 \\ 26 & -0.646325 \\ 27 & 0.035065 \\ 31 & -0.005654 \\ 32 & 0.181076 \\ 36 & -0.144575 \\ 40 & 0.219107 \\ 42 & 0.029306 \\ 46 & -0.063110 \\ 49 & 0.068013 \\ 54 & 0.180346 \\ 55 & 0.142062 \\ 56 & 0.166956 \\ 59 & -0.044833 \\ 61 & -0.666865 \\ 62 & -0.031473 \\ 65 & -1.295147 \\ 66 & -0.477632 \\ 69 & -0.720583 \\ 70 & -0.036968 \\ 72 & -0.058808 \\ 73 & -0.033974 \\ 74 & 0.075378 \\ 76 & 0.239545 \\ 77 & 0.024090 \\ 80 & -0.395309 \\ 85 & 0.129223 \\ 87 & -0.028252 \\ 89 & -0.212254 \\ 90 & 0.103341 \\ 91 & -0.061808 \\ 92 & 0.220380 \\ 99 & -0.031658 \\ 100 & 0.172818 \\ 103 & 0.110132 \\ 104 & -0.033148 \\ 105 & -0.131227 \\ 107 & 0.037901 \\ 110 & -0.034310 \\ 111 & -0.033043 \\ 112 & 0.064192 \\ 113 & -0.036392 \\ 116 & -0.038089 \\ & \end{array}$

MULTI PLI CADORES DE LAGRANGE DAS RESTRI ÇOES DE I GUALDADE DELTA P

$\begin{array}{cl}\text { BARRA } & \text { LAMB E } \\ 2 & 0.007405 \\ 3 & 0.009647 \\ 4 & 0.032077 \\ 5 & 0.033788 \\ 6 & 0.021529 \\ 7 & 0.018822 \\ 8 & 0.034873 \\ 9 & 0.043939 \\ 10 & 0.053177\end{array}$




$\begin{array}{ll}11 & 0.018761 \\ 12 & 0.015300 \\ 13 & 0.014510 \\ 14 & 0.015144 \\ 15 & 0.021799 \\ 16 & 0.018028 \\ 17 & 0.033396 \\ 18 & 0.024476 \\ 19 & 0.022053 \\ 20 & 0.022958 \\ 21 & 0.026294 \\ 22 & 0.032377 \\ 23 & 0.045044 \\ 24 & 0.043453 \\ 25 & 0.061421 \\ 26 & 0.058254 \\ 27 & 0.028575 \\ 28 & 0.023758 \\ 29 & 0.021139 \\ 30 & 0.036453 \\ 31 & 0.022085 \\ 32 & 0.026860 \\ 33 & 0.024788 \\ 34 & 0.033445 \\ 35 & 0.031816 \\ 36 & 0.031608 \\ 37 & 0.035849 \\ 38 & 0.038703 \\ 39 & 0.019651 \\ 40 & 0.014219 \\ 41 & 0.011849 \\ 42 & 0.018670 \\ 43 & 0.027688 \\ 44 & 0.027555 \\ 45 & 0.030175 \\ 46 & 0.041141 \\ 47 & 0.050157 \\ 48 & 0.049280 \\ 49 & 0.054765 \\ 50 & 0.045051 \\ 51 & 0.032897 \\ 52 & 0.028654 \\ 53 & 0.026968 \\ 54 & 0.031351 \\ 55 & 0.030408 \\ 56 & 0.030805 \\ 57 & 0.034768 \\ 58 & 0.030488 \\ 59 & 0.053225 \\ 60 & 0.061104 \\ 61 & 0.063485 \\ 62 & 0.061123 \\ 63 & 0.057821 \\ 64 & 0.062466 \\ 65 & 0.069256 \\ 66 & 0.072605 \\ 67 & 0.064433 \\ 68 & 0.071793 \\ 69 & 0.074550 \\ 70 & 0.044604 \\ 71 & 0.043645 \\ 72 & 0.041112 \\ 73 & 0.043186 \\ 74 & 0.036873 \\ 75 & 0.042293 \\ 76 & 0.036620 \\ 77 & 0.062806 \\ 78 & 0.061818 \\ 79 & 0.063990 \\ 80 & 0.075632 \\ & \\ 37 & \end{array}$




$\begin{array}{ll}81 & 0.073504 \\ 82 & 0.067762 \\ 83 & 0.073408 \\ 84 & 0.086329 \\ 85 & 0.095581 \\ 86 & 0.090485 \\ 87 & 0.091542 \\ 88 & 0.103495 \\ 89 & 0.114818 \\ 90 & 0.091520 \\ 91 & 0.091964 \\ 92 & 0.095950 \\ 93 & 0.083207 \\ 94 & 0.074583 \\ 95 & 0.069652 \\ 96 & 0.069531 \\ 97 & 0.071291 \\ 98 & 0.070679 \\ 99 & 0.069919 \\ 100 & 0.074494 \\ 101 & 0.080382 \\ 102 & 0.090404 \\ 103 & 0.058323 \\ 104 & 0.046771 \\ 105 & 0.041826 \\ 106 & 0.040806 \\ 107 & 0.028181 \\ 108 & 0.037075 \\ 109 & 0.035369 \\ 110 & 0.032943 \\ 111 & 0.040579 \\ 112 & 0.016254 \\ 113 & 0.031974 \\ 114 & 0.025706 \\ 115 & 0.025672 \\ 116 & 0.071786 \\ 117 & 0.009662 \\ 118 & 0.037348 \\ & \\ 91 & \\ 91 & 0\end{array}$

MULTI PLI CADORES DE LAGRANGE DAS RESTRI ÇOES DE I GUALDADE DELTA Q

$\begin{array}{cc}\text { BARRA } & \text { LAMB E } \\ 2 & -0.000414 \\ 3 & -0.000564 \\ 5 & -0.000339 \\ 7 & -0.000027 \\ 9 & 0.003143 \\ 11 & -0.000827 \\ 13 & -0.001699 \\ 14 & 0.000891 \\ 16 & 0.000372 \\ 17 & 0.002827 \\ 20 & 0.001199 \\ 21 & 0.001681 \\ 22 & 0.002603 \\ 23 & 0.003303 \\ 24 & 0.004330 \\ 28 & -0.000240 \\ 29 & -0.000116 \\ 30 & 0.003875 \\ 33 & 0.002948 \\ 34 & 0.005490 \\ 35 & 0.001005 \\ 37 & 0.006566 \\ 38 & 0.005536 \\ 39 & 0.001964 \\ 41 & -0.000432 \\ 43 & 0.009113\end{array}$




$\begin{array}{rr}44 & 0.011432 \\ 45 & 0.007310 \\ 47 & 0.001204 \\ 48 & 0.003423 \\ 50 & 0.000546 \\ 51 & 0.000190 \\ 52 & 0.000243 \\ 53 & -0.000257 \\ 57 & 0.000832 \\ 58 & 0.000330 \\ 60 & 0.000065 \\ 63 & -0.000108 \\ 64 & -0.000017 \\ 67 & -0.000081 \\ 68 & 0.000385 \\ 71 & 0.000242 \\ 75 & 0.000368 \\ 78 & -0.000368 \\ 79 & 0.000208 \\ 81 & 0.000949 \\ 82 & 0.003952 \\ 83 & 0.004144 \\ 84 & 0.000689 \\ 86 & -0.000309 \\ 88 & -0.000473 \\ 93 & -0.000894 \\ 94 & -0.000858 \\ 95 & -0.002062 \\ 96 & 0.000319 \\ 97 & -0.000075 \\ 98 & 0.000043 \\ 101 & -0.001183 \\ 102 & -0.000357 \\ 106 & -0.000301 \\ 108 & 0.000258 \\ 109 & 0.000198 \\ 114 & -0.000343 \\ 115 & -0.000383 \\ 117 & -0.001234 \\ 118 & -0.000602 \\ & \\ & 0\end{array}$

MULTI PLI CADORES DE LAGRANGE DAS RESTRIOES DE I GUALDADE OBTIDAS COM VARIAVEIS DE FOLGA - REATIVOS

$\begin{array}{cc}\text { BARRA } & \text { LAMB I } \\ 4 & -0.000000 \\ 6 & -0.000000 \\ 8 & 0.000000 \\ 10 & -0.000000 \\ 12 & 0.000000 \\ 15 & 0.000000 \\ 18 & -0.000000 \\ 19 & -0.000000 \\ 25 & -0.000000 \\ 26 & 0.000000 \\ 27 & 0.000000 \\ 31 & 0.000000 \\ 32 & -0.000000 \\ 36 & 0.000000 \\ 40 & -0.000000 \\ 42 & 0.000000 \\ 46 & 0.000000 \\ 49 & 0.000000 \\ 54 & -0.000000 \\ 55 & -0.000000 \\ 56 & -0.000000 \\ 59 & 0.000000 \\ 61 & -0.000000\end{array}$




$\begin{array}{rr}62 & 0.000000 \\ 65 & 0.000000 \\ 66 & -0.000000 \\ 69 & -0.000000 \\ 70 & 0.000000 \\ 72 & -0.000000 \\ 73 & -0.000000 \\ 74 & -0.000000 \\ 76 & -0.000000 \\ 77 & 0.000000 \\ 80 & -0.000000 \\ 85 & 0.000000 \\ 87 & -0.000000 \\ 89 & 0.000000 \\ 90 & -0.000000 \\ 91 & -0.000000 \\ 92 & 0.000000 \\ 99 & -0.000000 \\ 100 & 0.000000 \\ 103 & 0.000000 \\ 104 & 0.000000 \\ 105 & 0.000000 \\ 107 & -0.000000 \\ 110 & 0.000000 \\ 111 & 0.000000 \\ 112 & 0.000000 \\ 113 & -0.000000 \\ 116 & -0.000001\end{array}$

\section{MULTIPLICADORES DE LAGRANGE DAS RESTRI ÇOES DE DESIGUALDADE}

$\begin{array}{cll}\text { BARRA } & \text { Vk inf } & \text { Vk sup } \\ 12 & 0.000002 & 0.000002 \\ 2 & 0.000002 & 0.000002 \\ 3 & 0.000002 & 0.000002 \\ 4 & 0.000001 & 0.000005 \\ 5 & 0.000001 & 0.000006 \\ 6 & 0.000002 & 0.000003 \\ 7 & 0.000002 & 0.000002 \\ 8 & 0.000001 & 0.000008 \\ 9 & 0.000001 & 0.000010 \\ 10 & 0.000001 & 0.000003 \\ 11 & 0.000002 & 0.000002 \\ 1 & 0.000002 & 0.000002 \\ 13 & 0.000003 & 0.000001 \\ 14 & 0.000002 & 0.000002 \\ 15 & 0.000002 & 0.000002 \\ 16 & 0.000002 & 0.000002 \\ 17 & 0.000001 & 0.000005 \\ 18 & 0.000002 & 0.000003 \\ 19 & 0.000002 & 0.000003 \\ 20 & 0.000003 & 0.000002 \\ 21 & 0.000003 & 0.000002 \\ 22 & 0.000002 & 0.000002 \\ 23 & 0.000001 & 0.000004 \\ 24 & 0.000001 & 0.000004 \\ 25 & 0.000001 & 0.000007 \\ 26 & 0.000001 & 0.000007 \\ 27 & 0.000002 & 0.000002 \\ 28 & 0.000002 & 0.000002 \\ 29 & 0.000002 & 0.000002 \\ 30 & 0.000001 & 0.000010 \\ 31 & 0.000002 & 0.000002 \\ 32 & 0.000002 & 0.000003 \\ 33 & 0.000002 & 0.000003 \\ 34 & 0.000001 & 0.000004 \\ 35 & 0.000001 & 0.000004 \\ 36 & 0.000001 & 0.000004 \\ & 0.0001\end{array}$




\begin{tabular}{|c|c|c|}
\hline 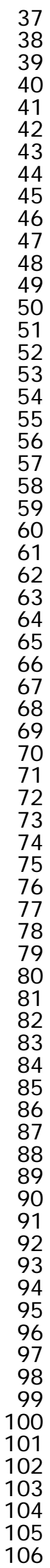 & 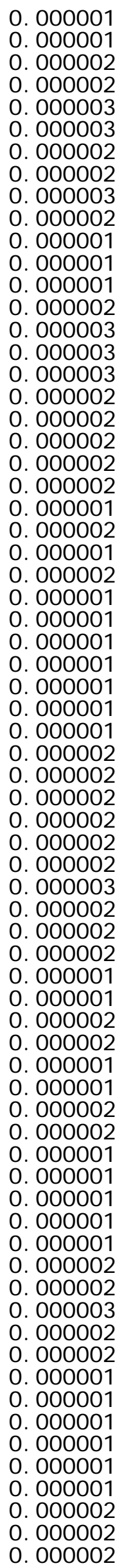 & 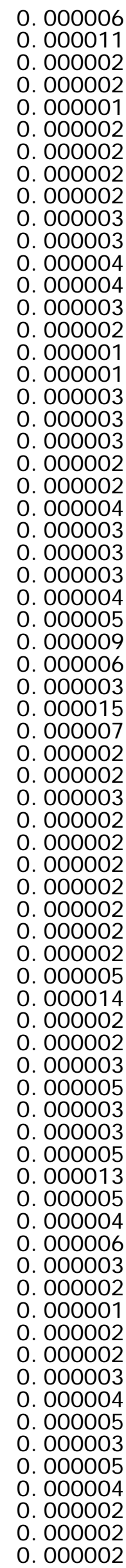 \\
\hline
\end{tabular}




$\begin{array}{lll}107 & 0.000002 & 0.000002 \\ 108 & 0.000002 & 0.000002 \\ 109 & 0.000002 & 0.000002 \\ 110 & 0.000002 & 0.000002 \\ 111 & 0.000002 & 0.000003 \\ 112 & 0.000003 & 0.000001 \\ 113 & 0.000001 & 0.000004 \\ 114 & 0.000002 & 0.000002 \\ 115 & 0.000002 & 0.000002 \\ 116 & 0.000001 & 0.000016 \\ 117 & 0.000004 & 0.000001 \\ 118 & 0.000003 & 0.000001\end{array}$

MULTIPLICADORES DE LAGRANGE DAS RESTRI ÇOES DE DESIGUALDADE OBTI DAS COM VARIAVEIS DE FOLGA

\begin{tabular}{|c|c|c|}
\hline $\begin{array}{l}\text { BARRA } \\
4 \\
6 \\
8 \\
10 \\
1 \\
15 \\
18 \\
19 \\
25 \\
26 \\
27 \\
31 \\
32 \\
36 \\
40 \\
42 \\
46 \\
49 \\
54 \\
55 \\
56 \\
59 \\
61 \\
62 \\
65 \\
65 \\
69 \\
70 \\
72 \\
73 \\
74 \\
76 \\
71 \\
80 \\
85 \\
87 \\
89 \\
90 \\
91 \\
91 \\
91 \\
91 \\
119 \\
100 \\
103 \\
104 \\
105 \\
10 \\
10\end{array}$ & 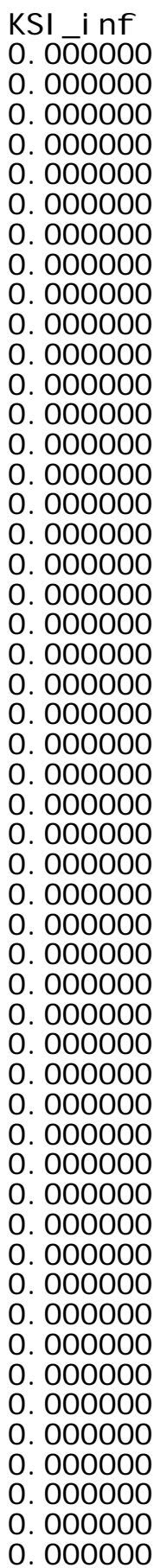 & 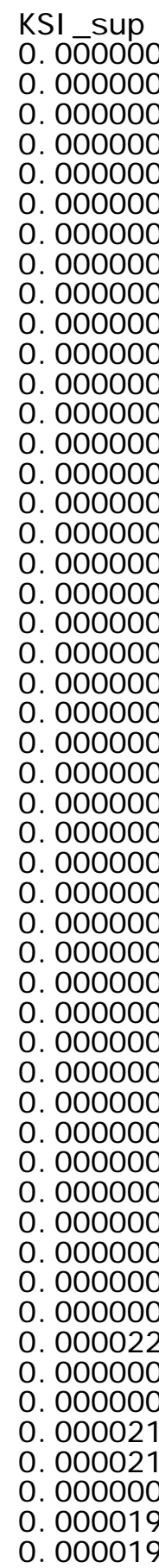 \\
\hline
\end{tabular}




\section{POTENCIA REATI VA GERADA}

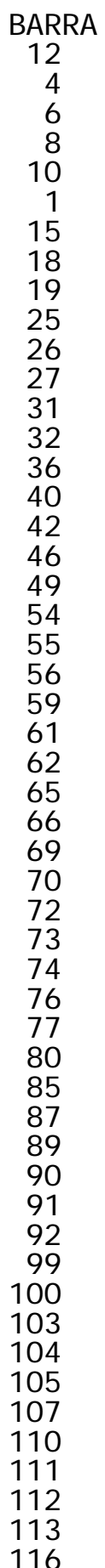

BARRA

\section{QMI N}

.9999 .000000

.50 .000000

$-50.000000$

$-50.000000$

.250 .000000

.50 .000000

$-50.000000$

$-50.000000$

$-50.000000$

250.000000

250.000000

$-50.000000$

.50 .000000

$-50.000000$

$-50.000000$

$-50.000000$

$-50.000000$

$-50.000000$

$-250.000000$

$-50.000000$

$-50.000000$

$-50.000000$

.50 .000000

.250 .000000

.50 .000000

$-250.000000$

.250 .000000

.250 .000000

.50 .000000

$-50.000000$

$-50.000000$

$-50.000000$

.50 .000000

.50 .000000

250.000000

.50 .000000

250.000000

250.000000

$-50.000000$

$-50.000000$

$-50.000000$

$-50.000000$

250.000000

.250 .000000

$-50.000000$

$-50.000000$

$-50.000000$

.50 .000000

.250 .000000

.50 .000000

$-50.000000$

.50 .000000
REATI VO E M MVAr

1. 263548

- 9.210264

- 2. 960957

$-17.617905$

$-137.085088$

2. 220739

$-7.711285$

$-8.069681$

11. 353220

$-9.163989$

$-64.631443$

3. 506293

- 0.565578

18. 106915

- 14.458318

21.909974

2. 930302

- 6.311475

6. 800973

18.033986

14. 205780

16. 694909

$-4.483554$

$-66.685718$

$-3.147514$

$-129.514649$

$-47.762122$

$-72.057889$

- 3.697181

$-5.880935$

- 3. 397469

7. 537207

23. 953979

2. 408536

$-39.530914$

12. 921906

$-2.825201$

- 21. 223771

10.333332

- 6.180786

22. 037470

- 3. 165945

17. 281890

11. 014936

- 3.314836

- 13.123009

3. 789904

$-3.430075$

- 3.301129

6. 418970

$-3.638572$

.3 .808960
QMAX

9999.000000

50.000000

50.000000

50.000000

250.000000

50.000000

50.000000

50.000000

115.000000

250.000000

250.000000

50.000000

50.000000

100.000000

50.000000

150.000000

50.000000

50.000000

250.000000

150.000000

150.000000

150.000000

50.000000

250.000000

50.000000

250.000000

250.000000

250.000000

50.000000

50.000000

50.000000

80.000000

100.000000

50.000000

250.000000

80.000000

250.000000

250.000000

100.000000

50.000000

100.000000

50.000000

250.000000

250.000000

0.000000

20.000000

60.000000

0.000000

0.000000

50.000000

0.000000

0.000000

TOTAL DE I NJEÇAO DE REATIVO DO SISTEMA $=-483.231442 \mathrm{MVAr}$ 
EVOLUÇAO DA FUNCAO OBJETIVO, DELTAP E DELTAQ POR I TERAÇAO - FLBCP

$\begin{array}{cccr}\text { I TERAÇAO } & \text { F. } 0 .(\text { e m MW) } & \text { MAX (DELTAP) } & \text { MAX(DELTAQ) } \\ 0 & 0.000000 & 607.000000 & 119.810000 \\ 1 & 117.736533 & 0.001318 & 0.000034 \\ 2 & 117.224972 & 0.001821 & 0.000057\end{array}$

EVOLUÇAO DA FUNCAO OBJETIVO, DELTAP E DELTAQ POR I TERAÇAO - FLBMP

$\begin{array}{cccr}\text { I TERAÇAO } & \text { F. O. (em MW) } & \text { MAX(DELTAP) } & \text { MAX(DELTAQ) } \\ 0 & 117.224972 & 0.001821 & 0.000057 \\ 1 & 116.811294 & 0.003798 & 0.000210 \\ 2 & 116.420025 & 0.005650 & 0.000347 \\ 3 & 116.030207 & 0.007412 & 0.000497\end{array}$

